\title{
Sequencing of sulfur metabolism gene promoters and identification of cadmium regulation in the promoter of a low affinity sulfate transporter from Brassica juncea
}

\author{
By \\ Justin Kicks, B. Sc. \\ A thesis submitted to \\ the Faculty of Graduate Studies and Research \\ in partial fulfillment of \\ the requirements for the degree of \\ Masters of Science \\ Biology Department \\ Ottawa-Carleton Institute of Biology \\ Carleton University \\ Ottawa, Ontario
}

August 2009 


\title{
Sequencing of sulfur metabolism gene promoters and identification of cadmium regulation in the promoter of a low affinity sulfate transporter from Brassica juncea
}

\author{
By \\ Justin Kicks, B. Sc. \\ A thesis submitted to \\ the Faculty of Graduate Studies and Research \\ in partial fulfillment of \\ the requirements for the degree of \\ Masters of Science \\ Biology Department \\ Ottawa-Carleton Institute of Biology \\ Carleton University \\ Ottawa, Ontario
}

August 2009 
The undersigned hereby recommend to

the Faculty of Graduate Studies and Research

acceptance of the thesis

\title{
Sequencing of sulfur metabolism gene promoters and identification of cadmium regulation in the promoter of a low affinity sulfate transporter from Brassica juncea
}

\author{
By \\ Justin Kicks \\ In partial fulfillment of the requirements \\ for the degree of Masters of Science
}

Chair, Department of Biology

Thesis Supervisor

Carleton University 


\section{ABSTRACT}

Brassica juncea is an ideal candidate for phytoremediation projects due to its fast growth rate and ability to tolerate and accumulate high concentrations of $\mathrm{Cd}$ in its shoots. The chelation of toxic metals, such as Cd, by sulfur-rich phytochelatins (PCs) is a common tolerance mechanism. Therefore, the PC biosynthetic pathway has recently been targeted for development of plant varieties with improved phytoremediative capacity. However, studies performed to date have focused on constitutive expression of the enzymes of this pathway and have resulted in stressed plants, compared to wild type, and little-to-no increase in $\mathrm{Cd}$ accumulation in the shoots. This study aims to investigate the transcriptional control of the $B$. juncea sulfur assimilation genes, which comprise the start of the phytochelatin biosynthetic pathway. Genome walking of the low affinity sulfate transporter (LAST) and two phytochelatin synthase (PS1 and PS2) genes provided segments $5^{\prime}$ of the translational start site for the creation of promoter:GUS constructs. Four truncations of the LAST promoter region were constructed to investigate two putative metal regulatory elements identified by bioinformatic analysis. The 5 LAST and 2 PS (PS1 and PS2) promoter-reporter constructs were employed for transformation of $A$. thaliana. Tissue-specific GUS expression, in the absence of $\mathrm{Cd}$, is controlled by a region between 436 and $1535 \mathrm{bp}$ of the LAST promoter and staining was localized to developing leaves after 3 weeks and the cauline leaves, flowers and siliques after 4 weeks. Increased GUS expression in the leaves of 2 and 3 week-old plants treated with $10 \mu \mathrm{M} \mathrm{Cd}$ suggest positive regulatory elements exist within 1535 bp of the LAST promoter. However, this pattern is not observed with $3771 \mathrm{bp}$ of the promoter region, suggesting negative elements are present between 2198 and $3771 \mathrm{bp}$ of the promoter region. 


\section{ACKNOWLEDGEMENTS}

Firstly, I would like to thank my former undergraduate supervisor, Sheila Macfie, for encouraging me to strive for grad studies early on in my career. Without her initial guidance and support I never would have made it to this day. I am doubly grateful to Susan Aitken for accepting me into her lab. Sues tireless strive for teaching and mentoring was a great inspiration. I am very thankful for the many oppurtunities she has provided and encouraged outside of my direct research in the past two years. I would also like to thank my advisors, Dr. Shelley Hepworth and Dr. Christiane Charest, for their support and ideas which have helped shape this project.

I have very much enjoyed my time here in the Biology Department at Carleton. The endless laughter and great conversations will be some of my fondest memories. In particular, I would like to thank Pratik Lodha, Nikita Rayne, Dominique Morneau, Heidi Los, Ally Jaworski, Faraz Quazi, Remmick So, Eman Ahmed-Muhsin and Christine Lowe for their company and companionship. Direct contribution to my project, whether helping to collect seeds or providing expertise and knowledge, has been kindly provided by Colleen Woodhouse, Brian Turnbull, Navya Kalidindi, Emily Hopwood and the Hepworth and Rowland labs. Also, the encouragement to participate in Lets Talk Science from Jen Skanes, Rob Smith and Michelle Zenko is greatly appreciated.

Last, but certainly not least, I am deeply grateful to my friends and family, who have made this journey enjoyable. I cannot possibly describe the inspiration and insight into life you have provided. Thanks to all my friends who came to visit me here in Ottawa. These have provided much needed breaks at times. Also, particular thanks to 
my hockey buddies Joe Stiles and Brad Riehl back home, and to my new teammates and friends in Ottawa for the good times in sport. 
3. DEDICATION

To My Parents 


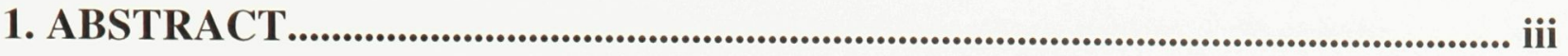

2. ACKNOWLEDGEMENTS............................................................................... iv

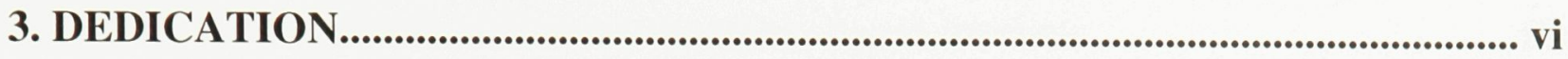

4. TABLE OF CONTENTS.......................................................................................... vii

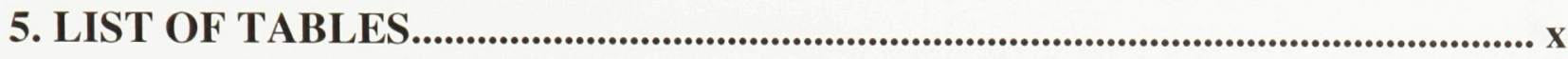

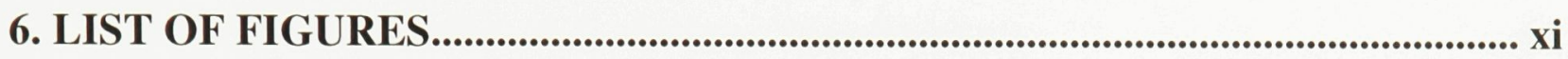

7. LIST OF ABBREVIATIONS............................................................................ xii

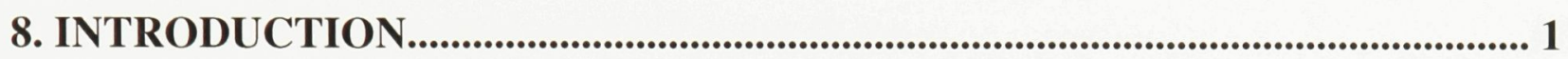

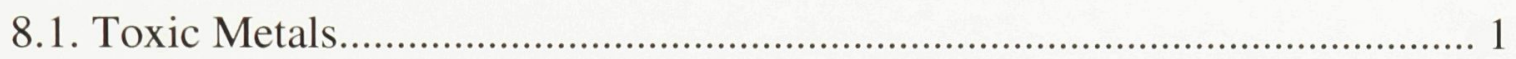

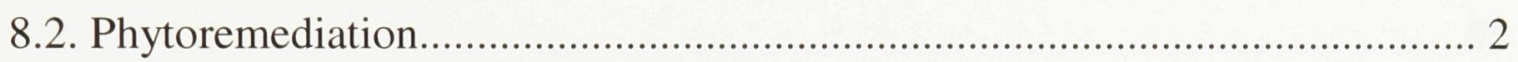

8.3. Cadmium Transport and Accumulation........................................................ 5

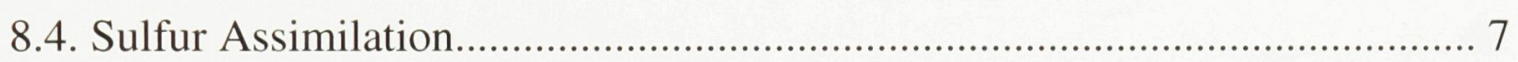

8.4.1 Sulfate transport: HAST/LAST _................................................ 9

8.4.2. Sulfate Activation: ATPS.............................................................. 10

8.4.3. Reduction of APS: APSR ......................................................... 12

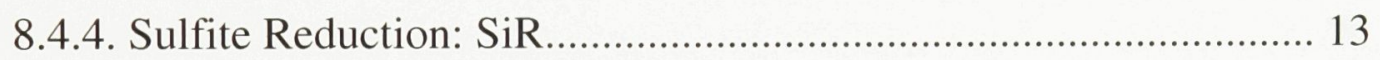

8.4.5. Cysteine Biosynthesis: OASS........................................................ 14

8.4.6. Glutathione Biosynthesis: GCL and GS...................................... 16

8.4.7. Glutathione Reduction: GR........................................................ 18

8.4.8. Phytochelatin Synthesis: PS.......................................................... 20

8.5. Toxic Metal Regulation of Promoters............................................................. 23

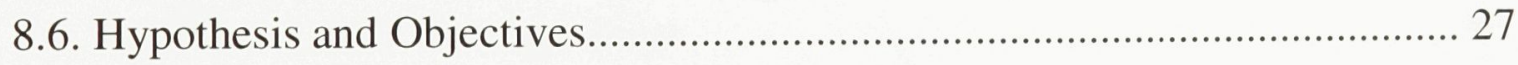

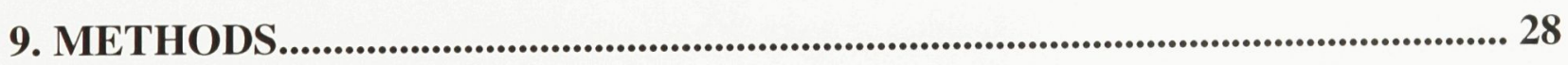

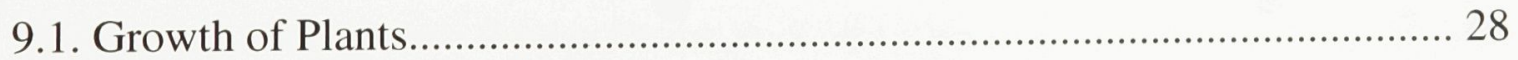

9.2. E. coli Transformation and Colony PCR..................................................... 30

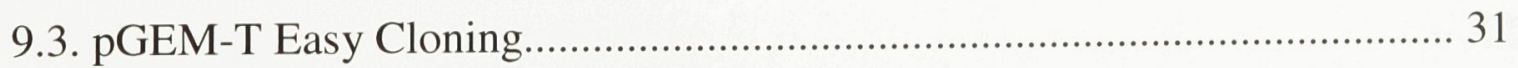

9.4. Genome Walker DNA libraries................................................................. 35

9.5. Primer Design for Genome Walking......................................................... 38

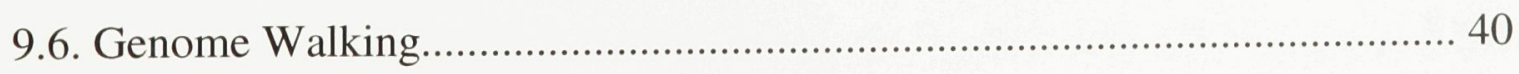




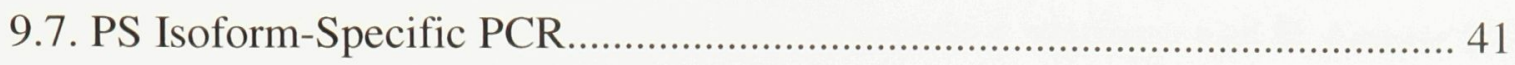

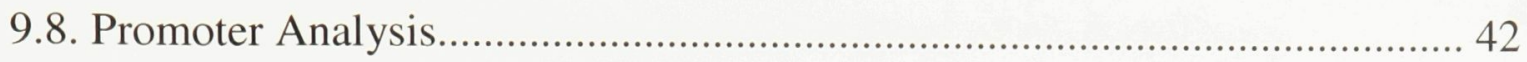

9.9. Promoter Cloning and Construction of Promoter-Reporter Plasmids.......... 44

9.10. Construction of LAST Promoter:gene Constructs in pBAR1 ..................... 50

9.11. Preparation of Electrocompetent Agrobacterium ................................... 53

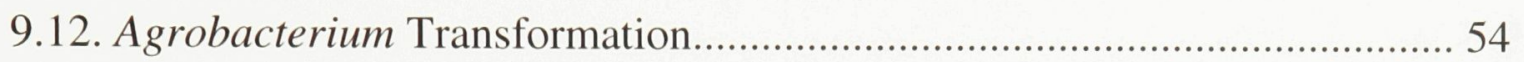

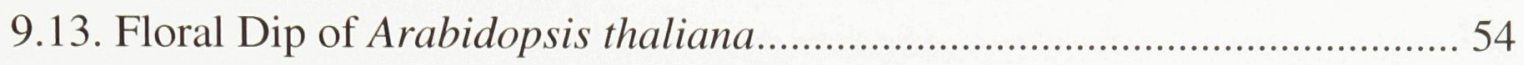

9.14. Basta Selection of A. thaliana Transformants.......................................... 56

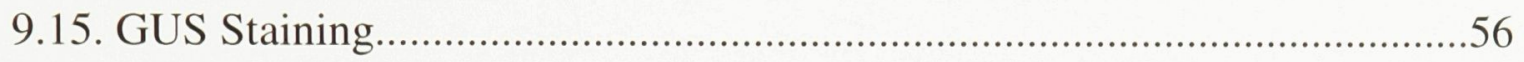

9.16. Sulfate-Limited and Cd Treated Plants............................................. 57

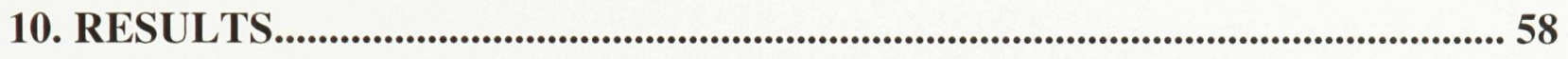

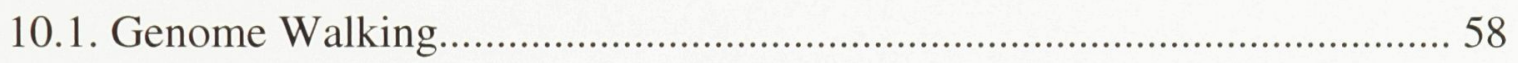

10.1.1. Genome Walking of ATPS............................................ 60

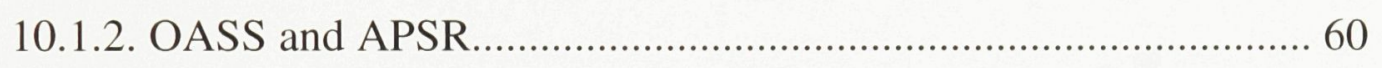

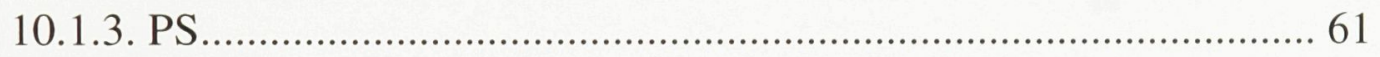

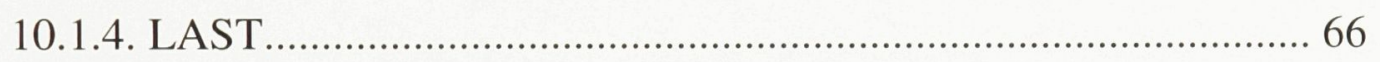

10.1.5. Bioinformatic Analysis of LAST Putative Promoter Region....... 66

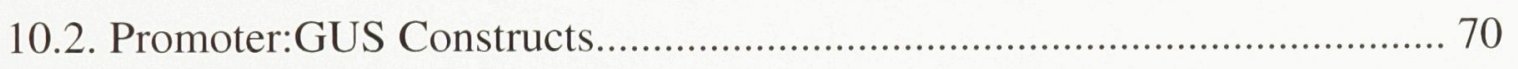

10.2.1. A. thaliana Transgenic Plants................................................ 71

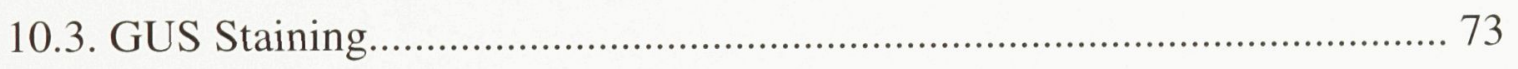

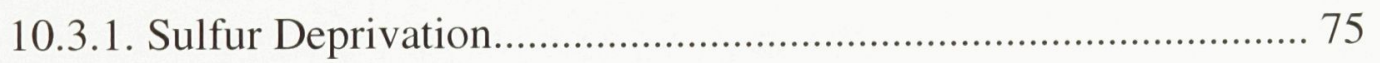

10.3.2. Cd Treatments............................................................. 76

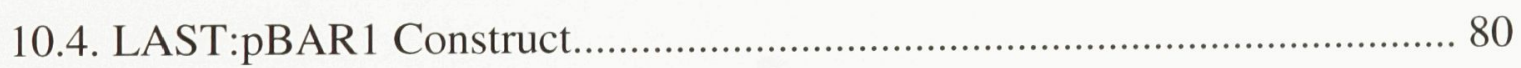

10.4.1. LAST Gene Cloning.................................................... 80

10.4.2. LAST Promoter Cloning for pBARI.................................... 81

10.5. Sultr2;1 and Sultr2;2 Expression in A. thaliana ................................. 84

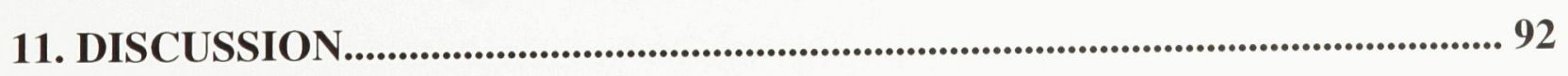

11.1. Evaluation of Genome Walking ..................................... 92

11.2. Identification of Additional PS Isoform............................................ 98

11.3. Construction of Promoter:GUS lines in A. thaliana ............................... 99

11.4. pLA:GUS Expression in A. thaliana ............................................... 101 
11.4.1. Comparisons of LAST Expression in A. thaliana and B. juncea 102 11.4.2. In silico and Experimental Evidence of cis-Regulatory

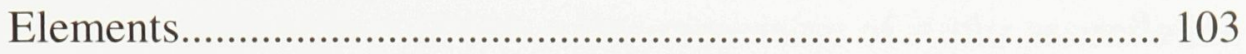

11.5. LA:pBAR1: Future Directions.................................................. 108

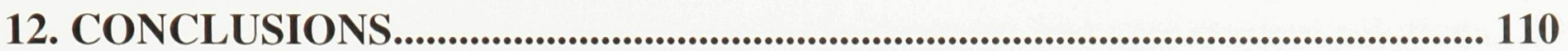

13. REFERENCES....................................................................................................... 112

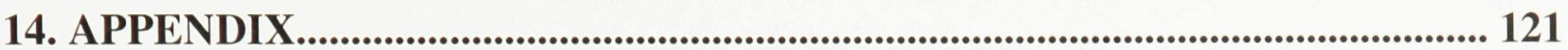




\section{LIST OF TABLES}

Table 1. Studies addressing the effect of $\mathrm{Cd}$ on the expression of sulfur assimilation and phytochelatin synthesis genes in A. thaliana and B. juncea. 25

Table 2. Nutrient salt concentrations for hydroponic and agar nutrient solutions.. 29

Table 3. Genome Walker Primers 39

Table 4. Primers designed for amplification of PS isoform-specific sequences of the coding region. 43

Table 5. Primers used for amplification of promoters from gDNA. 49

Table 6. Primers designed for amplification of LAST coding region from cDNA fragments.

Table 7. Length of promoter sequences obtained by genome walking 59

Table 8. Size of B. juncea low affinity sulfate transporter (LAST) promoter and truncations

Table 9. Number of transformant lines at each generation for $\mathrm{pLA}$ :GUS constructs 72

Table 10. AT content of sulfur assimilation gene promoter regions 94 


\section{LIST OF FIGURES}

Page

Figure 1. The enzymes and intermediates of sulfur assimilation and phytochelatin biosynthesis.

Figure 2. The TA cloning procedure for use of the pGEM T-Easy plasmid system.

Figure 3. Genome walking procedure 36

Figure 4. Construction of promoter-reporter constructs in the modified pGreen vector.

Figure 5. Genome walking and cloning of B. juncea PS1 and PS2 promoters 64

Figure 6. Selected results of bioinformatic promoter analysis and location of truncation primters for the $B$. juncea LAST promoter. 68

Figure 7. Representative GUS staining patterns of the (A) pLA:GUS, (B) pLATru4:GUS and (C) pLA-Tru3:GUS transgenic A. thaliana lines. 74

Figure 8. Effect of Cd treatment on wild-type A. thaliana. 77

Figure 9. GUS expression of the (A) pLA:GUS, (B) pLA-Tru4:GUS and (C) pLATru3:GUS transgenic $A$. thaliana lines treated with $10 \mu \mathrm{M} \mathrm{CdCl}{ }_{2}$ treatment 78

Figure 10. Genome walking and cloning of the $B$. juncea LAST gene..... 82

Figure 11. The developmental map expression of the A. thaliana LAST gene,

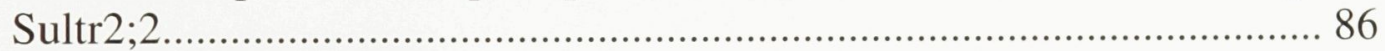

Figure 12. The developmental map expression of the A. thaliana LAST gene,

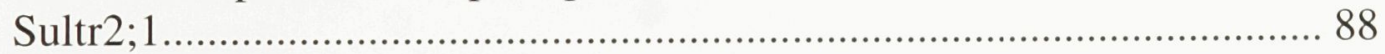

Figure 13. The developmental map comparing expression of the A. thaliana LAST genes, Sultr2;1 and Sultr2;2. 


\section{LIST OF ABBREVIATIONS}

\begin{tabular}{|c|c|}
\hline ABC & ATP binding cassette \\
\hline Amp & Ampicillin \\
\hline APS & Adenosine-5'-phosphosulfate \\
\hline APSK & APS kinase \\
\hline APSR & APS reductase \\
\hline ATM & $\mathrm{ABC}$ transporter of the mitochondria \\
\hline ATPS & ATP sulfurylase \\
\hline cDNA & Complementary DNA \\
\hline CaMV & Cauliflower mosaic virus \\
\hline CuRE & Copper response element \\
\hline CRR & Copper response regulator \\
\hline Cys & Cysteine \\
\hline $\mathrm{CO}$ & Constans \\
\hline DTT & Dithiolthretiol \\
\hline EDTA & Ethylenediamine tetraacetic acid \\
\hline GCL & Glutamate cysteine ligase \\
\hline gDNA & Genomic DNA \\
\hline GFP & Green fluorescent protein \\
\hline$\gamma$ Glu & $\gamma$-glutamyl \\
\hline Gly & Glycine \\
\hline GUS & $\beta$-glucuronidase \\
\hline GS & Glutathione synthase \\
\hline HAST & High affinity sulfate transporter \\
\hline HMT1 & Heavy metal transporter \\
\hline HMW & High molecular weight \\
\hline IPTG & Isopropyl- $\beta$ - $D$-thiogalactopyranoside \\
\hline IRT & Iron regulated metal transporter \\
\hline LAST & Low affinity sulfate transporter \\
\hline LB & Luria broth \\
\hline LMW & Low molecular weight \\
\hline MRE & Metal regulatory element \\
\hline NPT & Non-protein thiols \\
\hline OAS & $O$-acetylserine \\
\hline OASS & $O$-acetylserine sulfhydrylase \\
\hline PAPS & 3'-phosphoadenosine-5'-phosphosulfate \\
\hline parA & Protoplast auxin regulated A \\
\hline PC & Phytochelatin \\
\hline PCR & Polymerase chain reaction \\
\hline PDR & Pleiotropic drug resistance \\
\hline PS & Phytochelatin synthase \\
\hline SAT & Serine acetyl transferase \\
\hline SiR & Sulfite reductase \\
\hline Sultr & Sulfur transporter \\
\hline SR2 & Stress related 2 \\
\hline
\end{tabular}


Tris Tris-[hydroxymethyl]aminomethane

uidA E. coli $\beta$-glucuronidase gene

$\mathrm{X}$-gal

5-bromo-4-chloro-3-indolyl- $\beta$-D-galactoside

YCF1 Yeast cadmium factor 1

YOR1

Yeast oligomycin resistance 1

ZIP

ZRT/IRT-like proteins

ZNT1

Zinc transporter 1

ZRT

Zinc regulated metal transporter 


\section{INTRODUCTION}

\subsection{Toxic Metals}

Since the industrial revolution environmental contamination with toxic metals, such as cadmium $(\mathrm{Cd})$, has increased. Some metals, such as zinc $(\mathrm{Zn})$, copper $(\mathrm{Cu})$ and iron $(\mathrm{Fe})$ are essential micronutrients for biological organisms, serving as enzyme cofactors. These elements are required in small amounts and become toxic in larger quantities. Other metals, such as aluminum $(\mathrm{Al}), \mathrm{Cd}$, lead $(\mathrm{Pb})$ and mercury $(\mathrm{Hg})$ serve no biological function and are toxic even at low concentrations. Cadmium has been introduced to the environment as the result of its industrial applications in batteries, metal coatings, pigments and plastics, as well as from mining operations and the use of phosphate fertilizers (Nascimento and Xing, 2006; Nriagu, 1979). Cadmium accumulates in the food chain, thereby presenting a particular challenge in many countries, including India and China, where toxic metal contamination is widespread (Kramer, 2005). A recent study of agricultural soils irrigated with treated or untreated wastewater in India found $\mathrm{Cd}$, and other metals, to accumulate in the soil and crops. A number of the wastewater-irrigated sites were higher than the Indian standard of 3-6 mg Cd/kg (Sharma et al., 2008). Contamination is not limited to developing countries; a 1998 study estimated the Cd content of U.S. soils at between 100-345,000 $\mu \mathrm{g} \mathrm{Cd} / \mathrm{kg}$ soil, with the regulatory limit set at $100,000 \mu \mathrm{g} \mathrm{Cd} / \mathrm{kg}$ (Salt et al., 1998). There are also more than 50 000 localized, metal-contaminated sites in the United States alone (Bennett et al., 2003). The clinical manifestations of $\mathrm{Cd}$ exposure include kidney damage, neurological disorders and respiratory diseases (Minglin et al., 2005). In plants, Cd causes damage to photosystems I and II and interferes with the uptake and transport of the essential 
micronutrients Zn, Fe and Cu (Minglin et al., 2005; Shah and Dubey 1995). It may also displace $\mathrm{Zn}^{2+}$, calcium $\left(\mathrm{Ca}^{2+}\right)$, and $\mathrm{Fe}^{2+}$ ions in proteins, and inhibit antioxidative enzymes, such as catalase, ascorbate peroxidase and glutathione reductase, leading to a build-up of hydrogen peroxide and other reactive oxygen species in the cell (Schutzendubel and Polle, 2002).

\subsection{Phytoremediation}

The toxic effects of $\mathrm{Cd}$ and other metals have provided a selective pressure for plants to develop tolerance mechanisms. Metal tolerance can be achieved by exclusion or sequestration (reviewed in Hall, 2002). Exclusion can be facilitated by ectomycorrhizae, which surround plant roots and adsorb the metals on their cell walls or internalize and compartmentalize the metal, thereby limiting uptake by the plant. Binding of metals to the root cell walls and the active efflux of metals ions are also exclusion mechanisms (Hall, 2002). In contrast, sequestration involves the compartmentalization of metals in the plant vacuole, thus rendering them harmless in the metabolically-active cytosol (Hall, 2002). Species with the ability to sequester metal(s), or other contaminants, in their above-ground biomass in excess of the soil concentration, are referred to as hyperaccumulators. The ratio between the metal concentration in the plant shoots and the soil is referred to as the bio-concentration factor (McGrath and Zhao, 2003). Certain plant species, such as Thlaspi caerulescens (alpine penny cress) and Brassica juncea (Indian mustard), of the Brassicaceae family, and Pteris vittata (Brake fern), of the Pteridaceae family, are hyperaccumulators with bio-concentration factors up to 50-100 (McGrath and Zhao, 2003). However, this ability is rare in plants, with less than $0.2 \%$ of angiosperms being natural hyperaccumulators (McGrath, and Zhao, 2003). The 
hyperaccumulation of metals by plants may serve as a defense mechanism against pathogens and herbivory. For example, hyperaccumulation of nickel (Ni) in Streptanthus polygaloides (milkwort jewelflower) decreases the growth rate of fungal and bacterial pathogens (Boyd and Martens, 1994; Boyd et al., 1994). Additionally, in Niaccumulated Thlaspi montanum (penny cress), foliar feeding by Pieris rapae larvae was toxic (Boyd and Martens, 1994). Another experiment demonstrated decreased feeding in $\mathrm{Zn}$-accumulated plants by locusts, slugs and caterpillars in T. caerulescens (Pollard et al., 1997).

A variety of physical and chemical methods for the remediation of contaminated sites are available, including vitrification and storage as landfill (Ho et al., 1995). Phytoremediation is a cost-effective, biological alternative that employs hyperaccumulator plants to prevent leaching or remove toxic compounds from soil or water. Costs for conventional remediation methods are estimated at between $\$ 150$-350 per tonne, compared with $\$ 20-80$ per tonne for phytoremediation (Nascimento and Xing, 2006). Phytoremediation encompasses a variety of applications, including rhizofiltration, phytodegradation, phytostabilization and phytoextraction. The rhizofiltration process uses roots to absorb and adsorb pollutants, often from water or waste streams. The breakdown of organic compounds by plants is referred to as phytodegradation. Phytostabilization employs phreatophytic plant and tree species with extensive root systems, which exert hydraulic control to limit leaching of contaminants via groundwater and to stabilize contaminants in the rhizospere, thereby limiting their mobility. Phytoextraction, first suggested by Cheney in 1983, entails the removal of metals from soil through the use of hyperaccumulators, which sequester metals in their above-ground biomass (Cheney, 1983). 
The bioavailability of metals in soil is of particular challenge for phytoextraction. For example, metals such as chromium $(\mathrm{Cr})$, silver $(\mathrm{Ag})$ and tin $(\mathrm{Sn})$ are not soluble enough in soil to be bioavailable to plants. Other metals, such as $\mathrm{Pb}$, can heavily pollute a soil but are not taken up by plants because of low solubility and strong interactions with soil particles. There are two common approaches to phytoextraction: chelate assisted, and continuous. The former relies upon the application of chelators, such as ethylenediamine tetraacetic acid (EDTA), to the soil to release bound metals into the soil solution as bioavailable, chelate-metal complexes, which can be readily taken up by the plant. In continuous phytoextraction, the physiological attributes of hyperaccumulators are exploited to extract metals (Salt et al., 1998).

Phytoextraction can be extended by the use of biotechnology to design plants capable of accumulating and tolerating high metal contents. Since many hyperaccumulators are either slow growing or low-biomass plants, transferring genes involved in metal tolerance to non-hyperaccumulators with suitable agronomic properties may be an effective option for the development of phytoextraction. B. juncea has proven to be a good model species for the study of hyperaccumulation due to its relatively high biomass, compared to other hyperaccumulators, such as T. caerulescens, and its close relation to the model plant species Arabidopsis thaliana, also a member of the Brassicacae family. In addition, phytoremediation field studies have been conducted with B. juncea on selenium-contaminated sediment (Banuelos et al., 2005). 


\subsection{Cadmium Transport and Accumulation}

Cadmium is transported into the roots of plants via broad-specificity transporters, as exemplified by the ZNT1 (Zn transporter) protein, of the ZIP (ZNT/IRT-like protein) class of transporters, which has high affinity transport for $\mathrm{Zn}^{2+}$ and low affinity transport for $\mathrm{Cd}^{2+}$ in $T$. caerulescens (Pence et al., 2000). In $S$. cerevisaie, $\mathrm{Zn}^{2+}$ uptake activity by the A. thaliana ZIP1, ZIP2 and ZIP3 proteins, expressed in S. cerevisaie, is inhibited by $\mathrm{Cd}^{2+}$ (Grotz et al., 1998). The ZIP family also includes IRT1 (iron regulated metal transporter), which is responsible for most of the $\mathrm{Fe}^{2+}$ uptake into plants. The IRT1 protein can also transport divalent metal ions other than $\mathrm{Fe}^{2+}$ and $\mathrm{Cd}^{2+}$, such as $\mathrm{Zn}^{2+}$, manganese $\left(\mathrm{Mn}^{2+}\right)$ and cobalt $\left(\mathrm{Co}^{2+}\right)$ (Korshunova et al., 1999).

Plants have developed numerous mechanisms to cope with excess metal ions in the cytosol, including chelation and sequestration of metals by organic acids, metallothioneins, and phytochelatins (PCs) (Hall and Williams, 2003). Phytochelatins are sulfur-rich peptides of the structure $(\gamma \text {-Glu-Cys })_{n}$-Gly, where $n=2-11$, derived from glutathione (GSH), a key compound in the maintenance of the redox status of living cells. Therefore, the PC biosynthetic pathway is comprised of the enzymes glutamate cysteine ligase (GCL) and glutathione synthetase (GS), which produce GSH, and phytochelatin synthase (PS), which condenses GSH to form PCs (Cobbett and Goldsbrough, 2002). In plants, exposure to $\mathrm{Cd}^{2+}, \mathrm{Cu}^{2+}, \mathrm{Zn}^{2+}, \mathrm{Pb}^{2+}, \mathrm{Hg}^{2+}, \mathrm{Ni}^{2+}$, bismuth $\left(\mathrm{Bi}^{3+}\right), \mathrm{Ag}^{+}$, and gold $\left(\mathrm{Au}^{+}\right)$, or the anions $\mathrm{SeO}_{4}{ }^{-2}, \mathrm{SeO}_{3}{ }^{-2}$ and $\mathrm{AsO}_{4}{ }^{-3}$ results in an increase in phytochelatins levels (Grill et al., 1987; Speiser et al., 1992). Binding of $\mathrm{Cd}^{2+}, \mathrm{Ag}^{+}$and $\mathrm{Cu}^{2+}$ and of $\mathrm{Ag}^{+}, \mathrm{Hg}^{2+}$ and $\mathrm{Pb}^{2+}$ by $\mathrm{PCs}$ has been demonstrated in vivo and in vitro, respectively (Cobbett and Goldsbrough, 2002). Phytochelatins form both high molecular weight 
(HMW) and low molecular weight (LMW) complexes with Cd. In Schizosaccharomyces pombe, LMW-PCs are formed in the cytosol and transported into the vacuole, where HMW-PCs are produced. HMW-PC complexes have also been observed in the hyperaccumulator species B. juncea (Speiser et al., 1992).

Several ABC (ATP binding cassette) transporters have been shown to pump Cd complexes into the vacuole or to the exterior of yeast cells. For example, HMT1 (heavy metal transporter), which transports Cd-PC complexes into the vacuole, is required for Cd-tolerance in S. pombe (Ortiz et al., 1995), and Saccharomyces cerevisiae mutants lacking the YCF1 or YOR1 transporters are sensitive to Cd. The YCF1 (yeast cadmium factor) and YOR1 (yeast oligomycin resistance) proteins pump glutathione conjugates into the vacuole and outside of the cell, respectively (Li et al., 1996; Nagy et al., 2006). A. thaliana lines deficient in the AtATM3 (A. thaliana ABC transporter of the mitochondria) and AtPDR8 (A. thaliana pleiotropic drug resistance) ABC transporters have increased Cd-sensitivity compared to wild type plants (Kim et al., 2006; Kim et al., 2007). These transporters are mitochondrial and plasma membrane extrusion pumps, respectively (Martinoia et al., 2007). Although there are likely more transporter proteins involved in $\mathrm{Cd}$ detoxification, no transporters that pump $\mathrm{Cd}-\mathrm{PC}$ complexes into the plant vacuole have been identified to date. 


\subsection{Sulfur Assimilation}

The induction of PC biosynthesis by metal exposure causes an initial depletion of glutathione, followed by recovery and stabilization. This suggests that GSH synthesis is increased, with a corresponding requirement for increased sulfur assimilation (Fig. 1) (Mendoza-Cozatl et al., 2005). 
Sulfate Transporter (LAST/HAST)

$\mathrm{SO}_{4}{ }^{2-} \leadsto \mathrm{SO}_{4}{ }^{2-}$
ATP

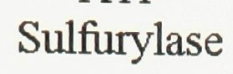<smiles>COP(=O)(O)OCC1OC(n2cnc3c(N)ncnc32)C(O)C1O</smiles>

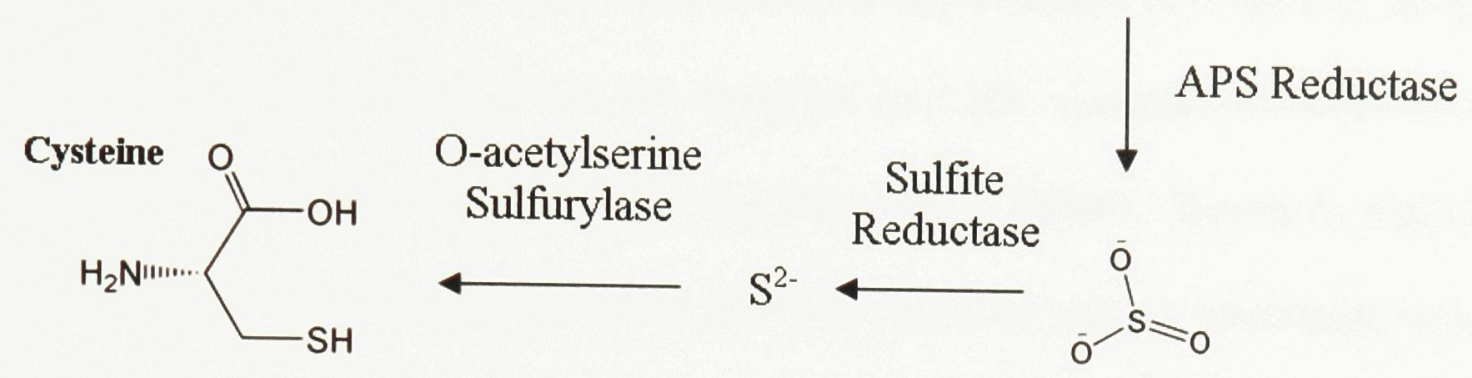

$\gamma$-Glutamylcysteine ligase

\section{$\gamma$-Glutamylcysteine}<smiles>N[C@@H](CCC(=O)N[C@@H](CS)C(=O)O)C(=O)O</smiles>

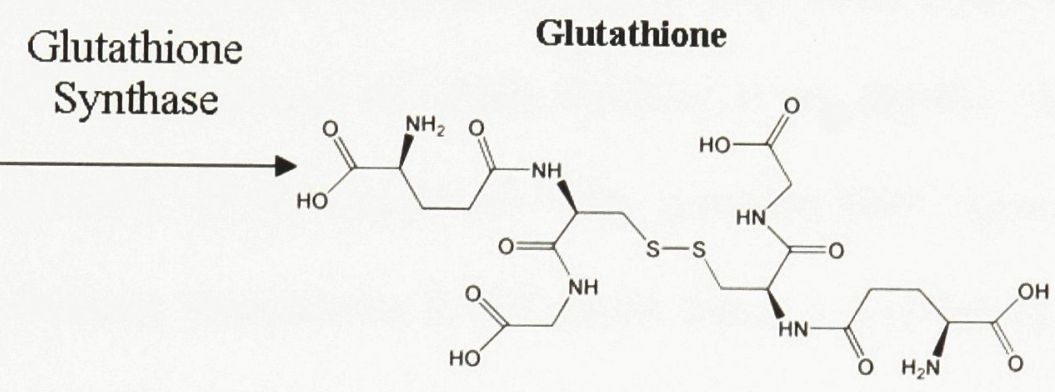

Phytochelatin
Synthase<smiles>CC(CCC(=O)NC(CS)C(=O)NC(C)C(=O)[O-])C(=O)[O-]</smiles>

Phytochelatin

Figure 1. The enzymes and intermediates of sulfur assimilation and phytochelatin biosynthesis. Low affinity sulfate transporter (LAST), high affinity sulfate transporter (HAST), adenosine-5' -phosphosulfate (APS). 


\subsubsection{Sulfate transport: HAST/LAST}

Plants take up sulfur as inorganic sulfate $\left(\mathrm{SO}_{4}{ }^{2-}\right)$, via high affinity and low affinity sulfate transporters (HASTs and LASTs, respectively). The HASTs have been implicated in the transport of sulfate from the environment into the root apoplast, while LASTs transport sulfate from the apoplast into the symplast of roots and from the vasculature into the cells of leaves (Buchner et al., 2004b). Seven A. thaliana sulfatetransporter genes have been cloned and characterized and an additional seven putative sulfate transporters have been identified in the genome (Yoshimoto et al., 2002; Buchner et al., 2004b). Of these, three appear to have strict regulatory roles in sulfate transport. The HAST Sultr1;1 (sulfate transporter) and Sultr 1;2 genes are expressed in the root tips and outer cell layers of the root (Yoshimoto et al., 2002; Buchner et al., 2004b). In contrast, analysis of promoter:GUS ( $\beta$-glucuronidase) and promoter:GFP (green fluorescent protein) reporter constructs demonstrate LAST genes Sultr2;1 and Sultr2;2 are expressed around vascular tissue in A. thaliana, thereby providing evidence of their role in sulfate transport from the symplast into the xylem. Specifically, Sultr2;1 is expressed in xylem parenchyma cells surrounding the xylem vessels and pericycle of the root and the xylem parenchyma and phloem cells in the root, while Sultr2;2 is expressed in the bundle sheath cells in the leaves (Takahashi et al., 2000). Therefore, the sulfate assimilation pathway of A. thaliana is thought to consist of sulfate uptake, from the soil into the root tips and cortical regions of the root, by the Sultr1;1 transporter, with subsequent transport into the xylem vessels, by the Sultr2;1 transporter, for transport to the shoot. Once in the xylem vessels of the leaves, sulfate is pumped from the xylem into the surrounding cells by the Sultr2;2 transporter. The expression levels of these genes are 
increased by sulfate starvation in A. thaliana (Takahashi et al., 2000). In B. juncea, which has a large demand for sulfur, sulfate uptake is predominately accomplished by low affinity transport during adequate sulfur conditions, and high affinity transport kinetics during sulfur deficiency (Ahmad et al., 2005). The transport kinetics were determined by quantifying the rate of uptake of radiolabeled sulfate by the roots of 10 day old B. juncea plants (Ahmad et al., 2005). Further analysis of LAST and HAST genes needs to be performed to identify their role in the changes in uptake kinetics. Treatment of $A$. thaliana with 5 and $50 \mu \mathrm{M} \mathrm{Cd} \mathrm{Cd}^{2+}$ both resulted in increased expression of the low affinity Sultr2;1 and Sultr2;2 genes in the roots, as determined by microarray (Herbette et al., 2006). In contrast, following exposure to $25 \mu \mathrm{M} \mathrm{Cd}^{2+}$, a $B$. juncea LAST gene, $88 \%$ and $67 \%$ identical to Sultr2;2 and Sultr2;1, respectively, showed a decrease in root expression (Heiss et al., 1999). The observed reduction in the expression of this LAST gene was proposed to maintain high sulfur content in the roots, by diminishing sulfate transport to other parts of the plant, to aid in metal detoxification. There are no other reports of the sequence or expression of other $B$. juncea HAST and LAST genes.

\subsubsection{Sulfate Activation: ATPS}

Once inside the cell, sulfate must be reduced to sulfide $\left(\mathrm{S}^{2-}\right)$ to be incorporated into biological compounds. This energetically costly process is accomplished via one of two species-specific pathways, which share an initial step that is common to all organisms studied, in which sulfate is activated to form adenosine 5'-phosphosulfate (APS) by ATP sulfurylase (ATPS) (Wray et al., 1998; Mendoza-Cozatl et al., 2005; Logan et al., 1996). Both chloroplast and cytosolic isoforms of ATPS have been 
identified in plants, with the majority of activity occurring in the chloroplast. Four isoforms of the gene encoding ATPS (ATPS1-4) have been identified in A. thaliana, all of which contain putative plastid transit peptides. Multiple isoforms have also been reported in soybean, Brassica napus and potato (Phartiyal et al., 2006). Although a cytosolic ATPS activity has been identified in A. thaliana (Rotte and Leustek, 2000; Hatzfeld et al., 2000), it appears to serve a role distinct from the plastidic activity, which is involved in the sulfate reduction pathway (Phartiyal et al., 2006). It remains unclear whether the cytosolic activity is due to one of the four isoforms with putative plastid transit peptides or if a fifth isoform exists (Rotte and Leustek, 2000). In maize, ATPS activity is highest in young leaves and in the elongation zone of the root (Phartiyal et al., 2006). Similarly, the activity of ATPS in the chloroplasts decreases with age in A. thaliana (Rotte and Leustek, 2000).

Northern blot analysis demonstrated that the expression of the A. thaliana ATPS3

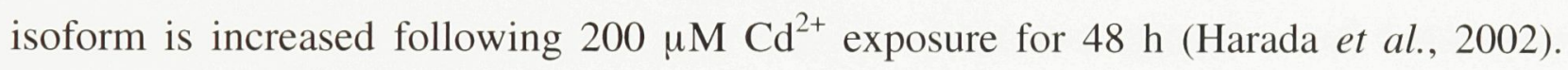
Root expression of ATPS was also found to be increased in a microarray study of $A$. thaliana treated with both high $(50 \mu \mathrm{M})$ and low $(5 \mu \mathrm{M})$ concentrations of $\mathrm{Cd}^{2+}$ for 2-30 hours (Herbette et al., 2006). Two isoforms of the gene encoding ATPS have been isolated from B. juncea: ATPS3 and ATPS6, which share highest sequence identity (87\% and 95.5\%) with the ATPS4 and ATPS1 isoforms of A. thaliana, respectively (Heiss et al., 1999). Northern blot analysis using coding-sequence and isoform-specific probes demonstrated that expression of B. juncea ATPS, although not the ATPS3 and ATPS6 isoforms, is increased in the roots and leaves of 13 day old plants upon $25 \mu \mathrm{M} \mathrm{Cd}^{2+}$ exposure for $48 \mathrm{~h}$, suggesting that, similar to A. thaliana, other Cd-responsive ATPS 
isoforms are present in B. juncea (Heiss et al., 1999).

\subsubsection{Reduction of APS: APSR}

Following the activation of sulfate by ATPS, the resulting APS is reduced to sulfite $\left(\mathrm{SO}_{3}^{-2}\right)$. In yeast and many bacterial species, APS is phosphorylated by APS kinase (APSK) to form 3'-phosphoadenosine-5' -phosphosulfate (PAPS), which is subsequently reduced to sulfite by PAPS reductase (Mendoza-Cozatl et al., 2005). Although homologs of APSK are also found in plants, they are involved in the sulfation of various secondary metabolites such as sulfated flavonols, glucosinolates, steroids and phytosulfokines rather than the reduction of sulfate to sulfide, the precursor of cysteine (Mendoza-Cozatl et al., 2005). In plants, the production of sulfite from APS is catalyzed by APS reductase (APSR), which relies on GSH as an electron donor. The observed protein-protein interaction between ATPS and APSR in Allium cepa (onion) suggests a mechanism for the channeling of APS between these two enzymes, and toward the pathway leading to cysteine biosynthesis (Cumming et al., 2007). The APSR enzyme contains two domains, both of which are required for APS reduction. Sequence alignment of APSR with thioredoxin indicates a thioredoxin-like structure in the Cterminal region of the protein, which is in keeping with the glutaredoxin activity of this enzyme, as GSH is the electron donor for the reduction of APS (Bick et al., 1998; Martin et al., 2005). Both the C-terminal domain and the iron-sulfur center, comprised by the Nterminal domain of the enzyme, are required for catalysis (Kopriva et al., 2001; Martin et al., 2005). The N-domain alone does not have APS reduction activity, but is thought to confer substrate specificity, as expression of the $\mathrm{N}$-domain as a separate protein in the 
presence of reductants other than the C-domain, such as thioredoxin, can restore APS reducing activity (Martin et al., 2005).

APSR activity is only detected in the chloroplast and the three isoforms of $A$. thaliana APSR contain putative plastid transit peptides (Rotte and Leustek, 2000). Microarray analysis has shown that expression of two of the A. thaliana APSR isoforms is increased upon exposure to 5 and $50 \mu \mathrm{M} \mathrm{Cd}^{2+}$ over a 2-30 hour period (Herbette et al., 2006). Southern blot analysis suggests that several APSR isoforms are also present in $B$. juncea, of which two (APSR2 and APSR8) have been sequenced (Heiss et al; 1999). Northern blot analysis, with a probe designed from, but not specific to, the APSR2 coding region, indicates the APSR expression is increased in roots and leaves in response to $48 \mathrm{~h}$ of a $25-\mu \mathrm{M}$ Cd treatment (Heiss et al., 1999). Minglin et al. (2005) employed differential display to determine that the expression of APSR2 is increased in leaves and roots following exposure to $200 \mu \mathrm{M} \mathrm{Cd}^{2+}$ for $48 \mathrm{~h}$.

\subsubsection{Sulfite Reduction: SiR}

The reduction of sulfite to sulfide by sulfite reductase ( $\mathrm{SiR}$ ) is common to bacteria, fungi and plants. This step requires the oxidation of three molecules of NADPH by $\mathrm{SiR}$. The $\mathrm{SiR}$ enzyme, like APSR, comprises a redox-active domain that contains an iron-sulfur cluster. The gene encoding $\mathrm{SiR}$ exists as a single copy in both Nicotiana tabacum and A. thaliana (Bork et al., 1998; Yonekura-Sakakibara et al., 1998) and no SiR sequence from $B$. juncea has been reported. The SiR enzyme, similar to ATPS and APSR, is localized to the chloroplast (Yonekura-Sakakibara et al., 1998). In S.

cerevisiae, expression of $\mathrm{SiR}$ is increased following treatment with $300 \mu \mathrm{M} \mathrm{Cd}^{2+}$ for $2 \mathrm{~h}$ 
(Momose and Iwahashi, 2001). The effect Cd exposure on SiR gene expression has not been investigated in plants.

\subsubsection{Cysteine Biosynthesis: OASS}

The condensation of sulfide and $O$-acetylserine (OAS) to form cysteine is catalyzed by the enzyme $O$-acetylserine sulfhydrylase (OASS). $O$-acetylserine is synthesized by serine acetyl transferase (SAT), which exists as a complex with OASS, referred to as cysteine synthase in plants. The activity of the two enzymes comprising the cysteine synthase complex has been detected in the chloroplast, cytosol and mitochondria of A. thaliana (Hesse et al., 1998). The OASS gene family in A. thaliana is comprised of three isoforms and one pseudogene: OASS A1 (cytosol), OASS B (plastid), OASS C (mitochondria) and OASS A2 (pseudogene) (Jost et al., 2000).

The expression of the cytosolic isoform of A. thaliana OASS A1 is increased following $1-3 \mathrm{~h}$ of exposure to $50 \mu \mathrm{M} \mathrm{Cd}^{2+}$, reaching a peak at $18 \mathrm{~h}$, and the in vivo OASS activity was also observed to increase over the same period. In situ hybridization demonstrated that the OASS A1 transcript increases in the cortex of the root and stem and in the vascular tissue of the stem and the leaf lamina upon $50 \mu \mathrm{M} \mathrm{Cd}^{2+}$ treatment for $18 \mathrm{~h}$. However, the cysteine concentration in the plant did not change in response to $\mathrm{Cd}$ exposure over this time frame. This is likely due to the increased GSH biosynthesis, for which cysteine is a substrate, as the GSH concentration peaks after 18 hours. Expression of OASS A1 under the control of the strong, constitutive cauliflower mosaic virus (CaMV) $35 \mathrm{~S}$ promoter in $A$. thaliana increased Cd tolerance as transgenic plants grow on agar media containing $250 \mu \mathrm{M} \mathrm{Cd}^{2+}$, a concentration at which wild-type plants failed to 
germinate. This suggests that the production of cysteine may be a rate limiting step in GSH production, and subsequent PC biosynthesis. This theory is supported by the observation that supplementation of wild-type $A$. thaliana plants with $200 \mu \mathrm{M}$ exogenous cysteine resulted in increased germination and leaf formation upon $250 \mu \mathrm{M} \mathrm{Cd^{2+ }}$ treatment, although to a lesser degree than observed for the CMV35S:OASS A1 transgenic plants (Dominguez-Solis et al., 2001).

In $B$. juncea, OASS is also encoded by a multi-gene family. Three isoforms have been identified and sequence alignment with the OASS A. thaliana isoforms suggests the presence of each of the chloroplast, cytosol and mitochondria localized isoforms (Schafer et al., 1998). Two studies have reported a transcriptional response of OASS to Cd treatment in B. juncea. Schafer et al (1998) observed an increase in total OASS

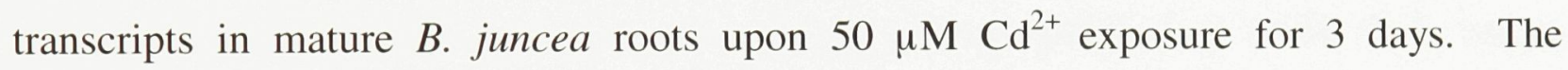
expression level of the mitochondria isoform was further analyzed using a unique probe, specific to the 3'-UTR, and no increase was observed during the same Cd treatment, suggesting that other isoforms are responsible for the increase in OASS expression. Fusco et al. (2005) performed a cDNA amplified fragment length polymorphism analysis (AFLP) of 6 week old B. juncea plants and observed an increase in OASS transcript levels after $10 \mu \mathrm{M} \mathrm{Cd}^{2+}$ treatments for 6 and $24 \mathrm{~h}$. The cDNA-AFLP technique relies on PCR-based amplification of cDNA fragments with arbitrary primers. The resulting products are resolved on an acrylamide gel and visualized for changes in band intensity. 


\subsubsection{Glutathione Biosynthesis: GCL and GS}

One of the most important metabolic fates of cysteine is incorporation into GSH, a redox-active tripeptide comprised of glutamate, cysteine and glycine ( $\gamma$ Glu-Cys-Gly). It is synthesized via two ATP-dependent enzyme-catalyzed reactions. In the first step, $\gamma$ glutamylcysteine ligase (GCL) catalyzes the condensation of glutamate and cysteine to form $\gamma$-glutamylcysteine. Glutathione synthase (GS) subsequently attaches glycine, via a peptide linkage, to the cysteine residue to form glutathione (Cobbett and Goldsborough, 2002). There are four structurally-distinct classes of GCL, each being unique to humans, yeast and protists, bacteria, and plants. A GSH binding site is present among the four GCL classes; however differences in structure suggest the sites evolved independently. The GCL step is considered a rate-limiting step in the formation of GSH and PC's due to the competitive inhibition by GSH. This inhibition is present in each of the GCL classes (Meister, 1995; Mendoza-Cozatl et al., 2005).

The PC content of two-week old A. thaliana plants was observed to increase from an undetectable amount to $400 \mu \mathrm{M}$ in response to an 18 -h treatment with $100 \mu \mathrm{M} \mathrm{Cd}^{2+}$. During this time, total glutathione concentration was initially depleted, after one hour, and rose back to wild-type cell concentrations by $18 \mathrm{~h}$. Northern blot analysis showed a corresponding steady increase in the transcript levels of GCL and GS over the 18 hours of Cd treatment (Xiang and Oliver, 1998). The observed reduction in the GSH/GSSG ratio over this 18 -h period may be a result of oxidative stress resulting from $\mathrm{Cd}$ treatment. The glutathione levels and GCL and GS expression levels showed similar patterns when plants were treated with $\mathrm{Cu}^{2+}$, although PC levels were not increased following this treatment (Xiang and Oliver, 1998). Increased expression of the B. juncea genes 
encoding GCL and GS has also been observed in response to exposure to $25 \mu \mathrm{M} \mathrm{Cd}^{2+}$ for up to 3 days (Schafer et al., 1998). In vitro studies of A. thaliana and B. juncea GCL indicate enzyme activity is controlled by the redox status of the cell. Mass spectrometry has identified two intramolecular disulfide bonds (Cys186-Cys406 and Cys349-Cys364) in A. thaliana GCL. Site-directed mutagenesis and subsequent kinetic analysis of the C186S, C406S, C349S and C364S mutants of A. thaliana GCL demonstrated that the C349-C364 disulfide bond is required for redox-sensitive activity (Hicks et al., 2007). In vivo analysis of the GCL enzyme in A. thaliana shows a shift from reduced GCL to the oxidized form upon $\mathrm{Cd}$ exposure, suggesting this redox activation occurs in response to metal toxicity (Hicks et al., 2007).

Genetic studies of GCL and GS have highlighted their important role in PC production and toxic metal tolerance. The introduction of tomato GCL, driven by the CaMV 35S promoter, complemented the cadmium sensitive cad2 A. thaliana mutant, which is deficient in GCL, by partially restoring Cd-tolerance (Cobbett et al., 1998 and Howden et al., 1995a). However, this construct did not increase the Cd tolerance of wild-type A. thaliana seedlings (Zhu et al., 1999b). In contrast, the expression of $E$. coli GCL driven by the CaMV $35 \mathrm{~S}$ promoter in $B$. juncea increased $\mathrm{Cd}$ tolerance and accumulation in seedlings and mature plants. These transgenic seedlings had up to 2-fold longer roots, compared to wild-type plants, when treated with $0.15,0.20$ or $0.25 \mathrm{mM}$ $\mathrm{Cd}^{2+}$ for 7 days. Mature transgenic plants treated with $0.05,0.075$ or $0.10 \mathrm{mM} \mathrm{Cd}{ }^{2+}$ for 14 days exhibited a less than 2 -fold increase in relative growth, measured as the whole plant biomass ratio between treated and untreated plants, compared to wild-type. Cdaccumulation was also significantly higher in leaves, by less than 2-fold, but not in the 
roots of transgenic plants, compared to wild-type, for the 0.05 and $0.10 \mathrm{mM} \mathrm{Cd}{ }^{2+}$ treatments, but not for $0.075 \mathrm{mM} \mathrm{Cd}^{2+}$. The transgenic seedlings showed elevated total amounts of GSH, PCs and non-protein thiols (NPT) in transgenic lines than wild-type plants. Glutathione concentrations increased 1.5-2.5 fold in untreated and $\mathrm{Cd}^{2+}$ treated $\left(0.00,0.15,0.20,0.25 \mathrm{mM} \mathrm{Cd}^{2+}\right)$ transgenic plants compared to wild-type, while NPT concentrations were 1.5 -fold greater for the $\mathrm{Cd}^{2+}$ treated transgenic plants but increases were not significant with untreated plants. A significant increase in PC concentration in transgenic plants compared to wild-type was also observed at $0.20 \mathrm{mM} \mathrm{Cd}$, but this increase was less than 2-fold (Zhu et al., 1999b). The same conditions were tested with transgenic B. juncea expressing the $E$. coli GS gene under the CaMV $35 \mathrm{~S}$ promoter. Seedling root length was significantly longer in the transgenic plants compared to wildtype at 0.15 and $0.20 \mathrm{mM} \mathrm{Cd}^{2+}$, but not at $0.25 \mathrm{mM} \mathrm{Cd}^{2+}$. The relative growth of mature transgenic lines was significantly higher than wild-type at each of the $\mathrm{Cd}^{2+}$ concentrations (0.05 or $0.10 \mathrm{mM} \mathrm{Cd}^{2+}$ ) tested. Shoot $\mathrm{Cd}$ concentration was also increased, by up to $22 \%$, in transgenic lines exposed to $0.1 \mathrm{mM} \mathrm{Cd}^{2+}$ for 14 days (Zhu et al., 1999a). These studies highlight the importance of GCL and GS in PC production and Cd tolerance.

\subsubsection{Glutathione Reduction: GR}

The balance between the levels of reduced and oxidized glutathione, GSH and GSSH, respectively, is maintained by glutathione reductase (GR). There are two genes encoding GR in A. thaliana, a cytosolic and organellar isoform (Xiang and Oliver, 1998). The reduced form of glutathione is involved in the maintenance of a reduced cellular environment, essential for biological reactions, and is required for the reduction of $\mathrm{H}_{2} \mathrm{O}_{2}$ 
by the ascorbate-glutathione cycle. Transition metals, such as $\mathrm{Zn}$ and Fe, cause direct oxidative stress through Fenton-type reactions. Although Cd cannot participate in Fenton reactions, it is believed to cause oxidative stress through indirect effects such as inhibition of the photosystems in the chloroplast. As a result of the transfer of electrons during photosynthesis, the chloroplast is a major source of reactive oxygen species, including $\mathrm{H}_{2} \mathrm{O}_{2}$, in plant cells (Noctor and Foyer, 1998). A study in which the E. coli GR gene was expressed, under the CaMV $35 \mathrm{~S}$ promoter, and targeted to chloroplast or cytosol in poplar found higher activity in the chloroplast-targeted plants (Foyer et al., 1995). In addition, photoinhibition, measured as $\mathrm{CO}_{2}$ assimilatory capacity, was significantly lower in the chloroplast-localized GR transgenic plants than in the wild-type and cytosol-localized GR transgenic plants (Foyer et al., 1995). A similar study expressing chloroplast and cytosol-localized E. coli GR in B. juncea found enzyme activity to be 20-50 times greater in the chloroplast localized GR lines than control plants, while the cytosol localized GR lines had only 2-4 fold greater activity (Pilon Smits et al., 2000). However, while the chloroplast-localized GR lines treated with 100 $\mu \mathrm{M} \mathrm{Cd}^{2+}$ for 14 days had significantly higher values for the chlorophyll fluorescence parameters, as measured by the variable fluorescence/maximum fluorescence ratio and photochemical quenching, when compared to wild-type plants, the transgenic GRexpressing lines exhibited no improvement in plant growth (Pilon-Smits et al., 2000). Glutathione reductase activity increases to a level 3-fold greater than control plants in a dose-dependent manner in mature B. juncea plants after 14 days treatment of 10, 20, 40, 80 and $160 \mu \mathrm{M} \mathrm{Cd}^{2+}$. However, when analyzed after 28 days an increase of between 2-3 fold in activity was only observed for $20 \mu \mathrm{M} \mathrm{Cd}^{2+}$ (Seth et al., 2008). 


\subsubsection{Phytochelatin Synthesis: PS}

The final step in PC synthesis is catalyzed by phytochelatin synthase (PS). The $A$. thaliana and wheat genes encoding PS were identified via cDNA library screening for genes that increased Cd-tolerance in Saccharomyces cerevisiae (Clemens et al., 1999; Vatamaniuk et al., 1999). The sequence of PS from several other plant species, including B. juncea, Thalspi caerulescens, Glycine max and Lotus japonica, as well as the yeast $S$. pombe and the nematode C. elegans, has subsequently been reported (Mendoza-Cozatl et al., 2005). A. thaliana possesses two distinct genes encoding PS and the L. japonica genome contains at least three (Ramos et al., 2007). The Cd-hypersensitive cadl A. thaliana lines lack or have reduced PC production, as determined by high pressure liquid chromatography (HPLC) analysis (Howden et al., 1995b). The expression of the PS gene varies among species and through development. For example, northern blot analysis demonstrated that in A. thaliana PS is constitutively expressed in the leaves and roots of mature, 21-day-old plants, and was unchanged by 6-h treatments with 25 and $100 \mu \mathrm{M}$ $\mathrm{Cd}^{2+}$ (Vatamaniuk et al., 1999). Similarly, 10 day-old A. thaliana plants treated with 1.5 $\mu \mathrm{M} \mathrm{Cd}^{2+}$ for 3 days maintained the same PS transcript levels in the leaves and roots as control plants, as determined by reverse transcriptase PCR (Ha et al., 1999). In contrast, reverse transcriptase PCR analysis of the wheat mRNA demonstrated a 5-10 fold increase in root PS transcript levels in 4-day-old seedlings treated with $100 \mu \mathrm{M} \mathrm{Cd}^{2+}$ for $6 \mathrm{~h}$ (Clemens et al., 1999). As well, increased expression of PS in 5-day-old A. thaliana seedlings grown on $50 \mu \mathrm{M} \mathrm{Cd}{ }^{2+}$ has been observed (Lee and Korban, 2002). Correspondingly, analysis of 5-day-old transgenic A. thaliana plants expressing a GUSreporter construct under control of a 2.0-kb fragment of the AtPS1 promoter region 
showed stronger staining when grown on $50 \mu \mathrm{M} \mathrm{Cd}^{2+}$, as compared to control, although 10 and 15-day-old plants did not differ from control plants when grown in the same $\mathrm{Cd}$ concentration (Lee and Korban, 2002). These studies suggest genetic regulation by $\mathrm{Cd}$ occurs only during early development in A. thaliana. In contrast, all three of the $L$. japonica PS genes show increased root expression in mature L. japonica plants after 100 $\mu \mathrm{M} \mathrm{Cd}{ }^{2+}$ treatment for 3, 6, 24 and $96 \mathrm{~h}$. The greatest increase in PS transcript was observed after $6 \mathrm{~h}$ of treatment $(2.1,2.9$ and 3.5-fold increase in expression for $L j \mathrm{PS} 1$, LjPS2 and LjPS3, respectively) (Ramos et al., 2007). In mature B. juncea plants, immunoblot investigation has indicated that the PS protein increases in the leaves but not in the roots after $120 \mathrm{~h}$ of exposure to $50 \mu \mathrm{M} \mathrm{Cd}^{2+}$ and that this increase is not due to higher mRNA levels, suggesting alternative mechanisms of regulation (Heiss et al., 2003).

Ectopic (non-native) expression of PS in various plants has provided insight into the activity and importance of this enzyme in Cd-tolerance. The expression of AtPS1 in B. juncea, driven by the CaMV $35 \mathrm{~S}$ promoter, increased Cd-tolerance in two of three independent lines tested. Seedling root length and shoot fresh weight were increased by up to $65 \%$ and 2 -fold, respectively, in the transgenic plants, as compared with the wildtype control, following a 10-day exposure to $100 \mu \mathrm{M} \mathrm{Cd}^{2+}$. However, the transgenic line that expressed the most PS protein, as determined by western blot, did not increase Cdtolerance and exhibited significantly lower root length than wild-type after the same Cdtreatment (Gasic and Korban, 2007). Similarly, expression of the gene AtPS1 by the CaMV 35 S promoter in A. thaliana was observed to cause Cd-hypersensitivity (Lee et $a l ., 2003)$. In this experiment, five independent transgenic lines were obtained, three of 
which produced twice the amount of PCs when 10-day-old plants were treated with 85 $\mu \mathrm{M} \mathrm{Cd}^{2+}$. These three lines all exhibited significantly shorter root length than wild-type when seeds were germinated and grown in 50 and $85 \mu \mathrm{M} \mathrm{Cd}^{2+}$ for 10 days. The other two lines, which produced approximately 50\% more PCs than wild-type, showed no difference in root length under Cd-treatments. All lines showed no difference in root length under control conditions. The observed hypersensitivity in the PS-expressing transgenic A. thaliana plants was proposed to be due to a decrease in the glutathione pool resulting from increased PC production. Correspondingly, NPT and GSH levels were significantly higher in transgenic plants than wild-type when treated with $85 \mu \mathrm{M} \mathrm{Cd}^{2+} . \mathrm{A}$ recent study by Wawrzynski et al. (2006) attempted to overcome the problem of glutathione supply by simultaneously expressing the E. coli SAT and GCL genes and the S. pombe PS gene, all under the CaMV 35 S promoter, in tobacco. The SAT, GCL and PS genes were selected because they are considered the rate limiting steps of cysteine, glutathione and PC synthesis, respectively. The resulting transgenic tobacco plants accumulated $122-167 \%$ more $\mathrm{Cd}$ in the roots than wild-type when 2 month old plants

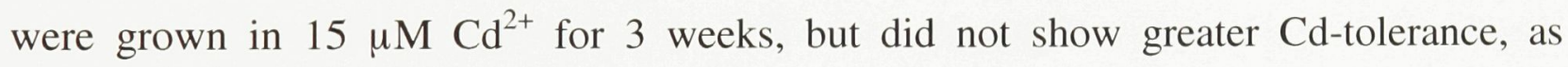
measured by fresh mass of roots and shoots. The root and shoot masses of the transgenic plants were decreased 2-fold compared to wild-type under control conditions. The results of this experiment have little relevance, from the perspective of phytoremediation, as the shoot-to-root ratio for Cd-accumulation was decreased, suggesting reduced translocation of $\mathrm{Cd}$ to the shoots. Another study has attempted to address this problem by expressing the AtPS1 gene under control of the leaf-specific chlorophyll a/b binding protein promoter in both wild-type and PS-deficient A. thaliana plants (Peterson and Oliver, 
2006). This approach alleviated Cd-stress in the roots, as root length was significantly higher in transgenic plants than wild-type when germinated and grown in 25 or $50 \mu \mathrm{M}$ $\mathrm{Cd}^{2+}$ for 10 days; however $\mathrm{Cd}$ accumulation in the shoots or roots was not increased as a result of these treatments.

\subsection{Toxic Metal Regulation of Promoters}

It is clear that the sulfur assimilation pathway plays an important role in $\mathrm{Cd}$ tolerance in plants (Table 1). Various studies also demonstrate that increasing the expression of genes in this pathway can lead to increased Cd-tolerance. However, the majority of these studies have relied upon strong, constitutive promoters, such as CaMV $35 \mathrm{~S}$, to drive the expression of the genes of interest. This has led to adverse effects, such as the Cd-hypersensitivity of PS-expressing A. thaliana plants, or only small improvements in Cd tolerance, which would not be sufficient for phytoremediation applications (Gasic and Korban, 2007, Zhu et al., 1999a, Zhu et al., 1999b). These effects are likely due to adversely changing the flux through the sulfur assimilation pathway, and related pathways, in the cell. For example, maintenance of the proper glutathione concentration and the balance between the reduced and oxidized forms in the cell are required to ensure stable redox status and altering this homeostasis will result in oxidative stress at the cellular level. Two recent studies have attempted to alleviate this problem by creating transgenics overexpressing multiple genes, or by using a tissuespecific promoter (Peterson and Oliver, 2006; Wawrzynski et al. 2006). However, strong constitutive promoters were used in both cases, with the result that the $\mathrm{Cd}$ tolerance and accumulation were not increased to a degree that would be useful for phytoremediation 
applications. Thus, more research into the regulation of this pathway is needed to develop a refined approach to increasing Cd-tolerance in plants. My project will address this issue by focusing on transcriptional control of genes of the sulfur assimilation pathway in B. juncea. By studying the regulation of this pathway in a hyperaccumulator species, we can identify potential genetic features that allow this species to tolerate $\mathrm{Cd}$ stress better than other species. 


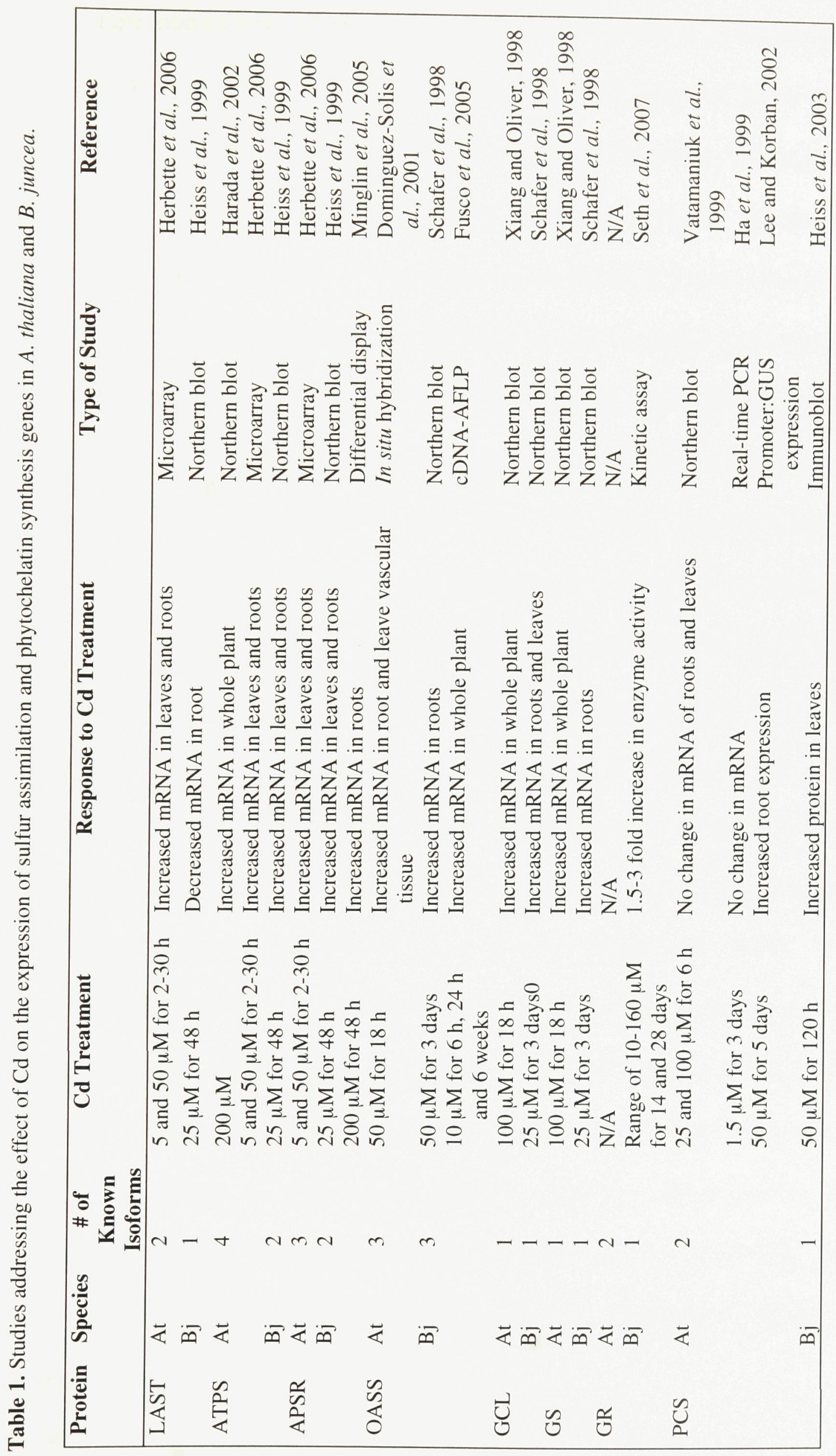


Few studies have addressed the cis-regulation of genes by transcription factors in response to toxic metals in plants, despite the identification of many metal-inducible genes. Metal regulatory elements (MRE) of yeast and animals are well characterized. For example, MRE regions are present in multiple copies in the promoters of metallothionein genes, such as the mouse metallothionein I gene (Searle et al., 1987). Metal-induced transcription factors bind to these sites to regulate the expression of these genes. In plants, a Cd-responsive, 29-bp element has been identified in the promoter region of the tobacco parA gene, an auxin regulated gene whose protein product is localized to the nucleus (Kusaba et al., 1996). The region was identified by assessing GUS activity of promoter:GUS reporter constructs transformed in tobacco protoplasts treated with $250 \mu \mathrm{M} \mathrm{Cd}{ }^{2+}$. In algae, copper response elements (CuRE) have been characterized (Quinn and Merchant, 1995). These elements respond to copper deficiency, but the CuRE and the corresponding transcription factor CRR1 are also required for gene induction by the presence of $\mathrm{Ni}^{2+}$ and $\mathrm{Co}^{2+}$ ions (Quinn et al., 2003). Recently, MRE-like sequences have been identified in the promoter of the metal induced gene Phaseolus vulgaris stress related 2 (PvSR2) (Qi et al., 2007). Expression of promoter:GUS reporter and truncation constructs in tobacco protoplasts and seedlings identified $\mathrm{Hg}^{2+}(15 \mu \mathrm{M})$-induced elements. Induction by several metals $\left(\mathrm{Cu}^{2+}, \mathrm{Hg}^{2+}\right.$, $\left.\mathrm{Cd}^{2+}, \mathrm{Zn}^{2+}, \mathrm{Mn}^{2+}, \mathrm{Co}^{2+}, \mathrm{Pb}^{3+}, \mathrm{Ag}^{+}\right)$was also observed. A similar approach was adopted in the present study, by targeting the promoter regions of the B. juncea LAST and PS genes and creating promoter:GUS fusion constructs, in the closely related model organism A. thaliana, for the analysis of their expression profiles and metal responsiveness. 


\subsection{Hypothesis and Objectives}

Based on the results of studies probing the expression patterns of genes of the phytochelatin biosynthetic pathway in response to $\mathrm{Cd}$ exposure and the effect of ectopic expression of several of these genes on Cd tolerance, I hypothesize that the LAST, ATPS, APSR, OASS and PS genes are spatially and/or temporally transcriptionally regulated by the presence of $\mathrm{Cd}$ in $B$. juncea. Since $A$. thaliana is closely related to $B$. juncea, the elements in these promoter sequences are expected to exhibit similar regulation in the two species. The understanding of this regulation will allow engineering of plants capable of accumulating and/or tolerating high amounts of metals for phytoremediation purposes. The main objectives of my M.Sc. project were to:

1. Obtain promoter sequences for genes of ATPS, APSR, OASS and PS from B. juncea. This work was carried out in collaboration with Colleen Woodhouse (M.Sc. candidate)

2. Construct promoter:GUS constructs of targeted genes, following the acquisition of sufficient sequence 5 ' of the translational start site. The pLAST:GUS constructs were made by Brian Turnbull (Honours thesis 2008)

3. Analyze the GUS expression patterns of LAST:GUS constructs in $A$. thaliana. 


\section{METHODS}

\subsection{Growth of Plants}

Brassica juncea seeds were germinated on moistened filter paper in the dark for two days prior to being transferred to a support matrix of sand supplemented, every other day, with an appropriate nutrient solution (Table 2). Plants were grown under controlled conditions in $22^{\circ} \mathrm{C}$ growth chambers with $24 \mathrm{~h}$ illumination at a light intensity of 90-160 $\mu \mathrm{mol} /\left(\mathrm{m}^{2} \mathrm{~s}\right)$. At one week, the seedlings were transferred from sand to mason jars containing $800 \mathrm{~mL}$ of nutrient solution (Table 2). Water was added, as required, to compensate for loss due to transpiration and nutrient solutions were replaced at 5-day intervals. Plants were grown in Hoagland's nutrient solution for genomic DNA (gDNA) extraction while the sulfur-insufficient solution was used for RNA extraction to clone the LAST coding sequence. The sulfur-sufficient solution was used as a control to ensure that any stress experienced by plants in the sulfur-insufficient nutrient solution was due to the lack of sulfur and not a result of any other differences in nutrients compared to the standard Hoagland's solution.

Arabidopsis thaliana (Col-0 wild type) seeds were vernalized at $4^{\circ} \mathrm{C}$ in the dark for 3 days on $0.8 \%$-agar plates containing AT (A. thaliana) nutrient solution (Table 2). The plates were transferred to $22^{\circ} \mathrm{C}$ growth chambers with $24 \mathrm{~h}$ illumination with a light intensity of $90-160 \mu \mathrm{mol} /\left(\mathrm{m}^{2} \mathrm{~s}\right)$. The transgenic $\mathrm{T}_{0}$ and $\mathrm{T}_{2}$ plants were transfered from plates to soil after 7-10 days after vernalization. 
Table 2. Nutrient salt concentrations for hydroponic and agar nutrient solutions

\begin{tabular}{|c|c|c|c|c|}
\hline & \multicolumn{4}{|c|}{ Working concentration $(\mu \mathrm{mol} / \mathrm{L})^{a}$} \\
\hline Nutrient & Hoagland's (50\%) & AT Media & Sulfur Sufficient & Sulfur Insufficient \\
\hline $\mathrm{MgSO}_{4}$ & 1000 & 2000 & 1000 & 50 \\
\hline $\mathrm{KH}_{2} \mathrm{PO}_{4}$ & 2000 & 1250 & & \\
\hline $\mathrm{NH}_{4} \mathrm{H}_{2} \mathrm{PO}_{4}$ & & & 1000 & 1000 \\
\hline $\mathrm{KNO}_{3}$ & 5000 & 6250 & 3000 & 3000 \\
\hline $\mathrm{Ca}\left(\mathrm{NO}_{3}\right)_{2}$ & 5000 & 2000 & 2000 & 2000 \\
\hline $\mathrm{Na}_{2}$ EDTA & 9 & 22.5 & & \\
\hline $\mathrm{FeSO}_{4}$ & 9 & 22.5 & & \\
\hline NaFeEDTA & & & 20 & 20 \\
\hline $\mathrm{H}_{3} \mathrm{HBO}_{3}$ & 46.25 & 46.25 & 25 & 25 \\
\hline $\mathrm{MnCl}_{2}$ & 9.15 & 9.15 & 2 & 2 \\
\hline $\mathrm{ZnCl}_{2}$ & 0.8 & 0.8 & 2 & 2 \\
\hline $\mathrm{CuSO}_{4}$ & 0.3 & 0.3 & & \\
\hline $\mathrm{CuCl}_{2}$ & & & 0.5 & 0.5 \\
\hline $\mathrm{Na}_{2} \mathrm{MoO}_{4}$ & 0.12 & 0.12 & & \\
\hline$\left(\mathrm{NH}_{4}\right)_{6} \mathrm{Mo}_{7} \mathrm{O}_{24}$ & & & 0.5 & 0.5 \\
\hline $\mathrm{KCl}$ & & & 50 & 50 \\
\hline
\end{tabular}

${ }^{\mathrm{a}}$ Micronutrients are below the line dividing the table 


\subsection{E. coli Transformation and Colony PCR}

A 10 -mL culture of E. coli strain DH10B (Invitrogen) in Luria-Bertani broth (LB) media ( $1 \% \mathrm{w} / \mathrm{v}$ tryptone, $1 \% \mathrm{w} / \mathrm{v} \mathrm{NaCl}, 0.5 \% \mathrm{w} / \mathrm{v}$ yeast extract), started from a glycerol stock, was incubated at $200 \mathrm{rpm}$ overnight at $30^{\circ} \mathrm{C}$. This culture was used to inoculate 1 L of LB media, which was incubated under the same conditions until the $\mathrm{OD}_{600}$ reached 0.5. The 1-L culture was chilled on ice for $10 \mathrm{~min}$ before centrifugation at $4000 \mathrm{rpm}$ at $4^{\circ} \mathrm{C}$ for $10 \mathrm{~min}$ in autoclaved centrifuge bottles. The cells were washed twice in $100-\mathrm{mL}$ volumes of ice-cold, filter-sterilized TB buffer (10 mM HEPES, pH 6.7, $15 \mathrm{mM} \mathrm{CaCl}_{2}$, $250 \mathrm{mM} \mathrm{KCl}$ ). The washed cell pellet was resuspended in $20 \mathrm{~mL}$ of $\mathrm{TB}$ buffer, containing $7 \%$ glycerol. The cells were incubated on ice for 10 minutes and stored as $300-\mu \mathrm{L}$ aliquots at $-80^{\circ} \mathrm{C}$.

The heat shock method was used for transformation of E. coli cells (Mandel and Higa, 1970). Competent cells $(100 \mu \mathrm{L})$ and no more than $200 \mathrm{ng}$ of DNA ligation product were mixed gently by pipetting and incubated on ice for $40 \mathrm{~min}$, followed by heat shock at $42^{\circ} \mathrm{C}$ for $45 \mathrm{~s}$. The mixture was incubated on ice for a further $2 \mathrm{~min}, 900 \mu \mathrm{L}$ of $\mathrm{LB}$ media was added and the culture was incubated at $37^{\circ} \mathrm{C}$ for 1 hour at $200 \mathrm{rpm}$. Cells were plated on LB agar plates, containing appropriate antibiotics, and incubated at $37^{\circ} \mathrm{C}$ for $\sim 18$ hours. Transformants were screened by colony PCR. The $100-\mu \mathrm{L}$ reactions contained $50 \mathrm{mM} \mathrm{KCl,} 10 \mathrm{mM}$ Tris- $\mathrm{HCl}, \mathrm{pH} 8.5,0.15 \%$ (v/v) Triton X, $2.5 \mathrm{mM} \mathrm{MgCl}_{2}$, 5\% (v/v) glycerol, $0.2 \mathrm{mM}$ dNTP's, $14 \mu \mathrm{g} / \mathrm{mL}$ Taq polymerase and $0.2 \mu \mathrm{M}$ of each primer. The Taq polymerase used in this experiment was purified from an E. coli expression vector (donated by Dr. Myron Smith, Carleton University, 2005). The theremocycler parameters were as followed: an initial 10-min denaturation step at $95{ }^{\circ} \mathrm{C}$, 
30 cycles of $1 \mathrm{~min}$ at $95^{\circ} \mathrm{C}, 1 \mathrm{~min}$ at $55^{\circ} \mathrm{C}, 2-4 \mathrm{~min}$ at $72{ }^{\circ} \mathrm{C}$, followed by a final extension step of $10 \mathrm{~min}$ at $72{ }^{\circ} \mathrm{C}$.

\section{3. pGEM-T Easy Cloning}

PCR amplicons required for sequencing or further use in construction of plasmids were sub-cloned by TA-cloning using the pGEM-T Easy cloning kit from Promega (Fig. 2). TA-cloning relies upon the natural tendency of Taq polymerase to add an extra adenine nucleotide to the 3' ends of amplicons during PCR. The pGEM-T Easy vector is provided linearized, from digestion with the restriction enzyme $E c o R V$, and subsequent T-tailing adds single 3' thymine nucleotide (T) overhangs. These 3' T overhangs allow ligation of PCR fragments containing adenine-nucleotide overhangs, such as those produced by Taq polymerase. The ligation reaction for insertion of PCR fragments into pGEM comprised: $1 \mathrm{X}$ rapid ligation buffer $(60 \mathrm{mM}$ Tris- $\mathrm{HCl}, \mathrm{pH} 7.8,20 \mathrm{mM} \mathrm{MgCl} 2,20$ mM DTT, 2 mM ATP, 10\% polyethylene glycol), 50 ng pGEM-T Easy vector, 90 ng of purified PCR products and 3 units of T4 DNA ligase, in a final volume of $10 \mu \mathrm{L}$. Ligation reactions were incubated at room temperature for $1 \mathrm{~h}$ and then overnight at $4^{\circ} \mathrm{C}$ before transformation of E. coli (strain DH10B) with $5 \mu \mathrm{L}(\sim 40-70 \mathrm{ng} / \mu \mathrm{L})$ of the ligated plasmid, via the heat shock method (Mandel and Higa, 1970). Cells were plated on LB agar plates containing $100 \mu \mathrm{g} / \mathrm{mL}$ ampicillin, $80 \mu \mathrm{g} / \mathrm{mL}$ 5-bromo-4-chloro-3-indolyl- $\beta$-Dgalactoside (X-Gal), and $0.5 \mathrm{mM}$ IPTG to enable blue/white screening, based on disruption of the $\beta$-galactosidase gene of the pGEM vector, for the identification of plasmids with inserts, and incubated at $37^{\circ} \mathrm{C}$ for $\sim 18$ hours. At least 10 white colonies per transformation were tested for presence of inserts by colony PCR with the pGEM-f 
(5'-CGA CGG CCA GTG AAT TGT AAT ACG) and pGEM-r (5'-CTC AAG CTA TGC ATC CAA CGC GT) primers, which flank the multiple cloning site of the vector. Cultures from colonies bearing plasmids with inserts of expected size were grown overnight in 5-10 mL of LB, with $100 \mu \mathrm{g} / \mathrm{mL}$ ampicillin, and plasmid was isolated using the Promega Wizard Plus SV Minipreps kit prior to sequencing (DNA Landmarks, Montreal, QC). 
Figure 2. The TA cloning procedure for use of the pGEM T-Easy plasmid system. The pGEM T-Easy plasmid (Promega) contains T-overhangs at the EcoRV restriction site within the LacZ gene. PCR products, which contain Aoverhangs produced by $T a q$ polymerase, are inserted at the $E c o R V$ site. Insertion of a segment of DNA disrupts the LacZ gene. The LacZ gene encodes $\beta$ galactosidase, which cleaves 5-bromo-4-chloro-3-indolyl- $\beta$-D-galactoside (X$\mathrm{Gal}$ ) to give a blue substrate. Therefore, blue colonies contain plasmid with an intact LacZ gene while white colonies contain plasmids with inserts. 

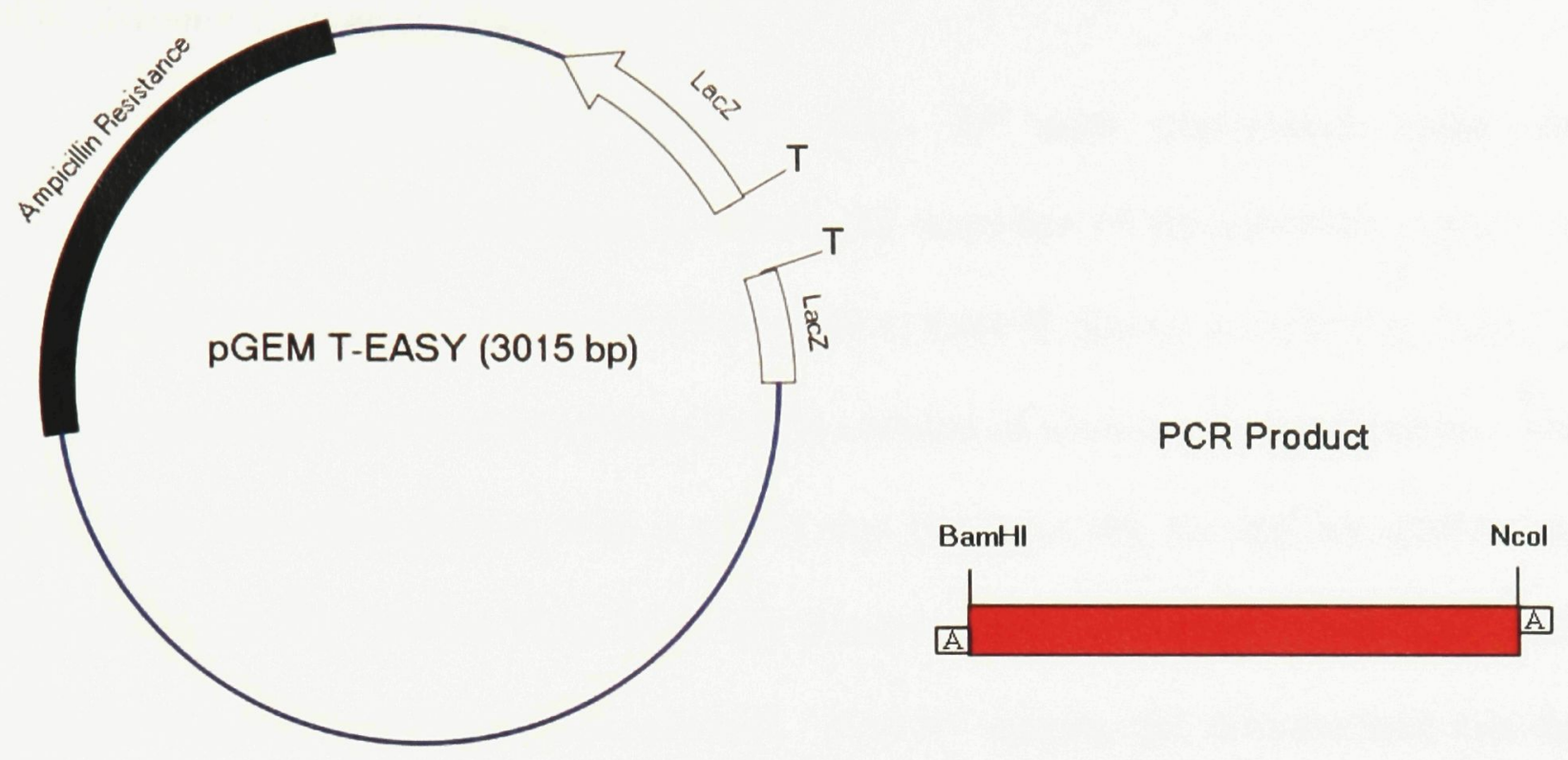

\section{Ligation}

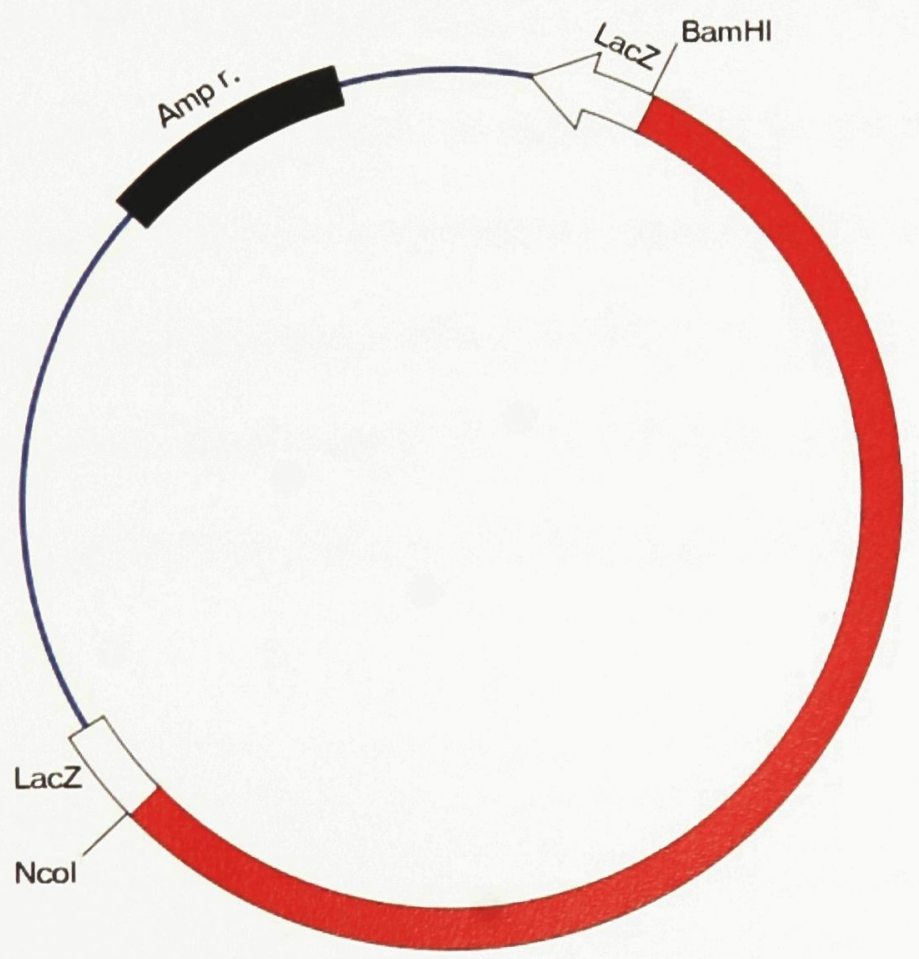




\subsection{Genome Walker DNA Libraries}

Genome Walker DNA libraries (Fig. 3) were constructed using the GenomeWalker kit from Clontech to obtain the sequence of the promoter regions of targeted $B$. juncea genes. Genomic DNA (gDNA) from B. juncea was purified from $1 \mathrm{~g}$ of new leaves of 3-week-old seedlings for the creation of Genome Walker libraries. The leaf tissue was homogenized with a mortar and pestle on dry ice and the gDNA was extracted with the DNeasy Plant Maxi Kit (Qiagen) and eluted with $1.8 \mathrm{~mL}$ Buffer AE (10mM Tris- $\mathrm{HCl}, 0.5 \mathrm{mM}$ EDTA, $\mathrm{pH} 9.0$ ). A $0.7 \%$ agarose gel demonstrated that the DNA averaged $20-25 \mathrm{~kb}$ in size. Genomic DNA was stored at $-80^{\circ} \mathrm{C}$ until the first usage, after which DNA was stored at $4^{\circ} \mathrm{C}$ to prevent shearing of gDNA by freeze/thawing.

Four Genome Walker DNA libraries were made from the B. juncea gDNA by digestion with distinct restriction endonucleases. Approximately $500 \mathrm{ng}$ of gDNA was digested overnight at $37^{\circ} \mathrm{C}$ with each of six restriction enzymes: $E c o R V\left(\mathrm{GAT}^{\wedge} \mathrm{ATC}^{\mathrm{A}}\right.$ ), DraI (TTT^^AAA), HpaI (GTT^ACC), BstZ17I (GTA^TAC), PsiI (TTA^ ${ }^{\wedge}$ TAA) and ScaI $\left(\mathrm{AGT}^{\wedge} \mathrm{ACT}\right)$, which cleave a six-base-pair recognition site to produce blunt ends. Enzymes with AT-rich recognition sites were selected, as plant DNA is AT rich (Yu et al., 2002). A $0.5 \%$ agarose gel was run to confirm digestion, compared to the undigested gDNA control. The GenomeWalker adaptor $\left(4.75 \times 10^{5} \mu \mathrm{mol}\right)$ was then ligated to the blunt ends of the digested gDNA with 1 unit of T4 DNA ligase per $20 \mathrm{ng}$ of gDNA. 
Figure 3. Genome walking procedure. A single Genome Walker library is created by first digesting gDNA with a single blunt-end cutting restriction enzyme (RE). The Genome Walker (GW) adapter segments (Clontech) are ligated to both ends of the digested fragments. A pair of gene-specific primers (gspl and gsp2) is designed from the known sequence of the targeted gene (indicated in red) for nested PCR with adapter primers (ap1 and ap2). The primary PCR reaction comprises the outer primer pair (gsp1 and ap1). The secondary PCR reaction uses the primary PCR product as template and is amplified with the inner primer pair (gsp2 and ap2). 

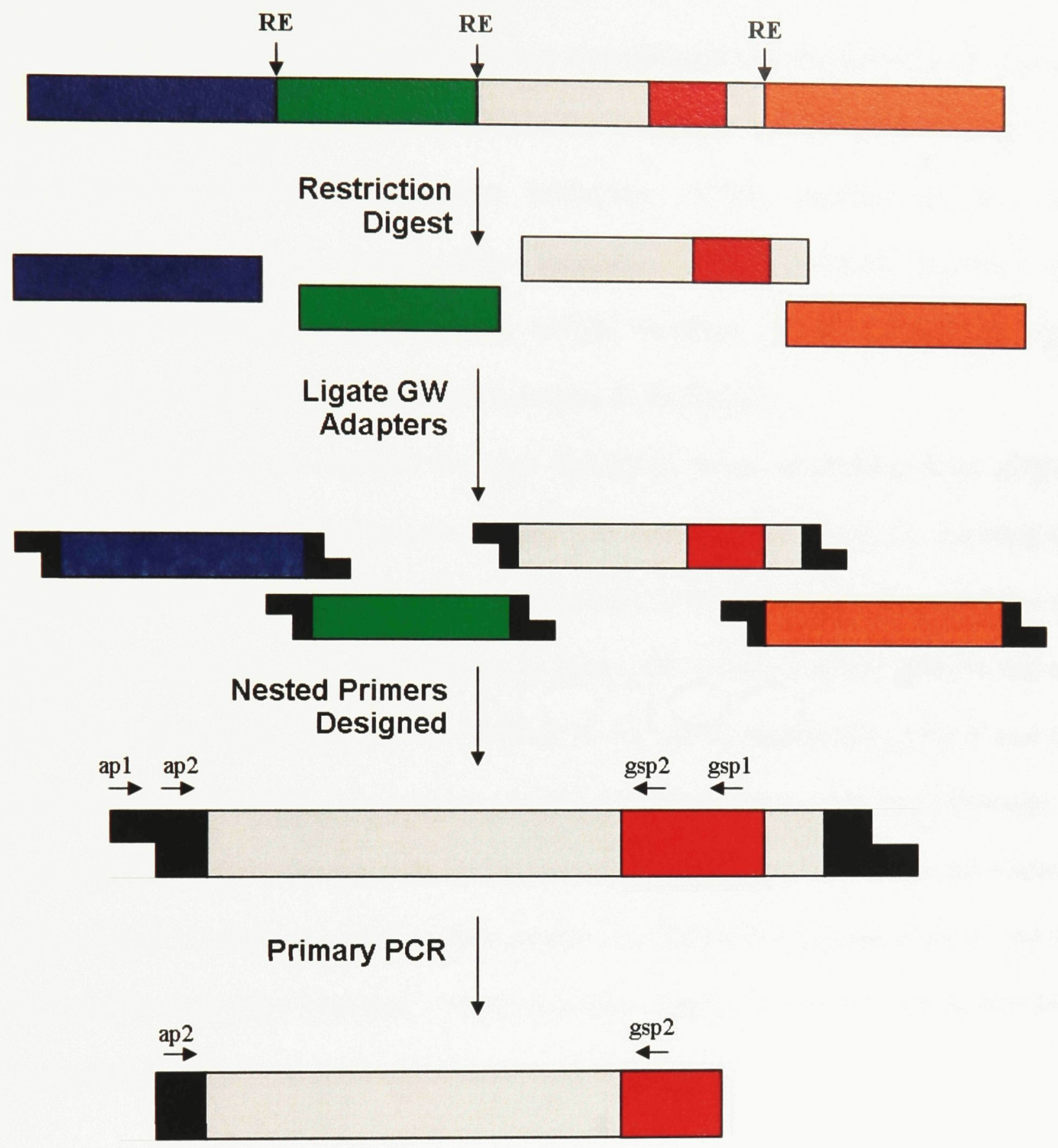

Secondary PCR

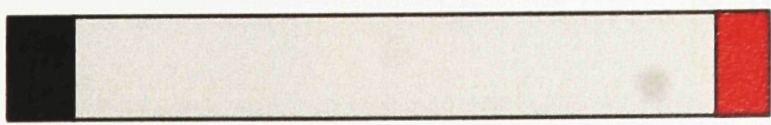




\subsection{Primer Design for Genome Walking}

Nested pairs of gene-specific primers were designed for the following B. juncea gene accessions (Table 3): the low affinity sulfate transporter (LAST, GenBank accession \#: AJ223495), adenosine triphosphate sulfurylase (ATPS, GenBank accession \#: AJ223499), adenosine-5'-phosphosulfate reductase (APSR, GenBank accession \#: AJ001207), $O$-acetylserine sulfhydrylase (OASS, GenBank accession \#:Y10845), and phytochelatin synthetase (PS, GenBank accession \#: AJ278627).

Nested PCR is required for high specificity when amplifying from gDNA libraries. The genome walking nested primer sets were designed (Table 5), according to the guidelines of the GenomeWalker kit (Clontech), to be between 26-30 nucleotides in length, to avoid sequences with hairpins, to have a GC content between $40-60 \%$ and to contain no more than 3 guanines or cytosines in the last six nucleotides of the 3 ' end of the primer. An annealing temperature of $67^{\circ} \mathrm{C}$ is optimal for use with the Advantage 2 Taq polymerase, although this was not possible in most cases due to the high AT content of the targeted sequences. Primers were located $\sim 50-100$ bp downstream of the 5' end of the available GenBank sequence. Primer sets were designed to be as close as possible without overlapping and none were further than 200 bp apart. 
Table 3. Genome Walker Primers

\begin{tabular}{|c|c|c|c|}
\hline Gene & Primer Name & $\begin{array}{c}\begin{array}{c}\text { Location } \\
\text { from }\end{array} \\
\text { ATG }^{a}\end{array}$ & Sequence \\
\hline \multirow[t]{5}{*}{ LAST } & LAST-gsp1 & 1503 & TTTGGCGACTTGACCGAGATGTTGACCGTT \\
\hline & LAST-gsp2 & 1384 & ACACGATTAGGGTTGCTAATATCACTG \\
\hline & LA4-1bB-gsp3 & -2040 & CACGGATTTCCAGACTGGTACCTTGAAC \\
\hline & LA4-1bB-gsp4 & -2138 & TGGACGTTCTAATCTCTCCCGTACTGCT \\
\hline & LA-gsp5 & -3727 & GGTCCTACTACACCAGCACTTTCTCCAT \\
\hline \multirow[t]{7}{*}{ ATPS } & ATPS-gsp2 & -57 & TTTGGTGAGTGGCTGAGAGAGGAAAGGA \\
\hline & ATPS-gspl & -117 & ACAACGGCGAGGTTTCGATGGGAACGA \\
\hline & AT3-2a-gsp3 & -201 & ATCCGAGATCTGGATCCGCTCCGAAA \\
\hline & AT3-2a-gsp4 & -239 & GGAGAGGACGAATCCGAATCCAGATAG \\
\hline & ATgsp6a & -2556 & GAAGGGTATGGAGTCTCTCACAATTGGC \\
\hline & ATgsp6b & -2736 & AGGATGGACTTACCTTCTATTTGCTGG \\
\hline & ATgsp6c & -2847 & GTTCCCATCTTCCGGTTGTCATCAGA \\
\hline \multirow[t]{6}{*}{ APSR } & APSR-gsp 1 & +70 & CAGAACAAGGTCCAGAGCGTGAGAA \\
\hline & APSR-gsp2 & +30 & GGCAGTTGAAGAAGAAGTGACAGCTA \\
\hline & AP2-1b-gsp5a & -89 & CCGCGTTAAATAATGGCCGACCGAAT \\
\hline & AP2-1b-gsp3 & -228 & TCTTGTGGTTGTATCGCGTGGTGAGCA \\
\hline & AP2-1b-gsp4 & -256 & TGGTGTGTGATATGTGGCGGCCTTGAAT \\
\hline & AP-gsp4b & -284 & TTGGAAATGACACACGGCTGGAATAAATCT \\
\hline \multirow[t]{8}{*}{ OASS } & OASS-gsp1 & +90 & ACGACCAACACATCCTTCAGCCACATTG \\
\hline & OASS-gsp2 & +59 & AGATACACCAATGGAGTGTTCCCAATCAA \\
\hline & OAgsp5a & -238 & TGGGAAGAGGAAGATGATGAGGATACC \\
\hline & OA2-4aA-gsp3 & -254 & TGAGGATACCTCGCGAGTGAGAAAGAGA \\
\hline & OA2-4aA-gsp4 & -350 & ACAGGTAGATGCCACATGCTTTGGATG \\
\hline & OAgsp5b & -266 & CGCGAGTGAGAAAGAGAATGAAGAAGC \\
\hline & OAgsp6a & -363 & TATAGGTTCGGTTCGATTCGGATCCAGG \\
\hline & OAgsp6b & -414 & AAGACCCGCAAGTTTGGATTCTGGTC \\
\hline \multirow[t]{2}{*}{$P S$} & PS-gsp 1 & +114 & TCCTTCCATAGTGCCTTTCTGAAGCG \\
\hline & PS-gsp2 & +53 & AAGTCAATGGCTGGAGGAGAAGGAAGA \\
\hline \multirow[t]{5}{*}{ PS1 } & PSgsp5a & -1020 & TTCTCCСТTGCAACCTCCACTCCAT \\
\hline & PS-gsp3b & -1132 & TGTGCTTTGCACGGTTCGTTTGTCAGTC \\
\hline & PSgsp5b & -1379 & GGTGTCTCTCTTTCTCTCTACCCAATCC \\
\hline & PS2-1bA-gsp3 & -1450 & CTTCACACCCATATTTCTTCCCGCTTCG \\
\hline & PS2-1bA-gsp4 & -1477 & GGAACCAGAATCAGGATCAGAATCTTGTAGA \\
\hline \multirow[t]{2}{*}{$P S 2$} & PSgsp8a & -913 & GGCATACAGTTTAGCACGCAATGTCAG \\
\hline & PSgsp8b & -970 & GCTCCCAGTTTGGTTTACGGAATCAG \\
\hline
\end{tabular}

${ }^{a}$ Numbers indicate location of primers with respect to the translational start site of the target gene. Negative numbers represent sites 5' of the translational start site, positive numbers represent sites 3' of the translational start site. 


\subsection{Genome Walking}

Nested PCR, using Advantage 2 polymerase (Clontech), was employed for amplification of targeted sequences from the genome walker libraries (Fig. 3). The product of the first reaction comprising adaptor primer 1 and the most 3' of the two genespecific primers, was used as the template for the second PCR reaction, comprising adaptor primer 2 and the $5^{\prime}$ of the two gene-specific primers. Each reaction comprised: Advantage 2 PCR buffer and Polymerase Mix (Clontech), $0.2 \mathrm{mM}$ dNTP, $0.2 \mu \mathrm{M}$ adapter primer 1 (Clontech), $0.2 \mu \mathrm{M}$ gene-specific primer, and $5 \mathrm{ng}$ of the genome walker library, in a $50 \mu \mathrm{L}$ reaction. The primary PCR reaction conditions consisted of an initial denaturation step at $94^{\circ} \mathrm{C}(2 \mathrm{~min})$, followed by 6 cycles of $94^{\circ} \mathrm{C}(25 \mathrm{~s})$ and $72^{\circ} \mathrm{C}(3 \mathrm{~min})$, and then 31 cycles of $94^{\circ} \mathrm{C}(25 \mathrm{~s})$ and $67^{\circ} \mathrm{C}(3 \mathrm{~min})$ followed by a final annealing/extension step of $7 \mathrm{~min}$ at $67^{\circ} \mathrm{C}$. The secondary PCR reaction conditions consisted of an initial denaturation step at $94^{\circ} \mathrm{C}(2 \mathrm{~min})$, followed by 4 cycles of $94^{\circ} \mathrm{C}(25$ s) and $72^{\circ} \mathrm{C}(3 \mathrm{~min})$, and then 19 cycles of $94^{\circ} \mathrm{C}(25 \mathrm{~s})$ and $67^{\circ} \mathrm{C}(3 \mathrm{~min})$ followed by a final annealing/extension step of $7 \mathrm{~min}$ at $67^{\circ} \mathrm{C}$. The reaction products were analyzed on a $1.5 \%$ agarose gel and amplicons from the secondary PCR greater than 500 bp were gel excised and purified (GFX kit, GE Healthcare).

Each purified DNA band was ligated to the pGEM-T Easy (Promega) TA cloning vector and sequenced (DNA Landmarks). New gene-specific primers were designed, as required, base on the sequence obtained to continue to 'walk' out into the promoter region. The sequences from successive amplifications were processed using VecScreen (NCBI) to remove pGEM vector sequence and compiled into full-length contiguous DNA sequences using the Cap3 sequence assembly program (http://pbil.univ- 
lyon1.fr/cap3.php) (Huang and Madan, 1999). Full-length promoter sequences were amplified via PCR directly from gDNA, ligated to pGEM and used to create promoterreporter constructs in the pGreen/pSoup expression system, as described in section 9.9.

\subsection{PS Isoform-Specific PCR}

Diagnostic amplifications of the PS1 and PS2 coding sequences were performed to test the presence of two isoforms. A primer was designed within conserved regions of the PS coding region and nested primers in non-conserved regions $\sim 50 \mathrm{bp} 5^{\circ}$ of the translational start site for PS1 and PS2, in the sequences derived from genome walking (Table 4). The primers within the coding region were designed by aligning the coding regions of 7 PS gene sequences, each from plants of the Brassicacea family (A. thaliana: AF085231, NM_100279, Arabidopsis halleri: AY463694, Brassica napus: AM265632, B. juncea: AB057416 T. caerulescens: AY540104, Thlaspi japonicum: AB085626). PCR reactions reactions contained 50-80 $\mathrm{ng}$ of $B$. juncea $\mathrm{gDNA}, 50 \mathrm{mM} \mathrm{KCl}, 10 \mathrm{mM}$ Tris$\mathrm{HCl}, \mathrm{pH} 8.5,0.15 \%$ (v/v) Triton X, $2.5 \mathrm{mM}, \mathrm{MgCl}_{2}, 5 \%$ (v/v) glycerol, $0.2 \mathrm{mM}$ dNTP's, $14 \mu \mathrm{g} / \mathrm{mL}$ Taq polymerase and $0.2 \mu \mathrm{M}$ of each primer. PCR amplicons were ligated to pGEM and sequenced. 


\subsection{Promoter Analysis}

The PLACE (Higo et al., 1999) database was used to identify putative cisregulatory elements present in the promoter sequences of targeted $B$. juncea genes and the corresponding genes from $A$. thaliana. The A. thaliana sequences (Sultr2;1: AT5G10180.1, Sultr2;2: AT1G77990.1) were obtained from The Arabidopsis Information Resource (TAIR) website (www.arabidopsis.org). Unique elements present in the $B$. juncea LAST promoter sequence were used as a basis to design truncations of the promoter. 
Table 4. Primers designed for amplification of PS isoform-specific sequences of the coding region

\begin{tabular}{|lcc|}
\hline Primer & $\begin{array}{c}{ }^{a} \text { Location } \\
\text { from ATG }\end{array}$ & Sequence \\
\hline PScDNA1 & +499 & GCCATATCTCTCTCAGCATTATAGCCACC \\
PS1-seq5 & -2 & CTTCCAGTGCGATCTTTGTCAC \\
PS1-seq6 & -50 & TCATCCCTCCAAGAAACCTGTCG \\
PS2-seq6 & -41 & TCTCATCACGCCCAAAGTTGAAAG \\
PS2-seq5 & -98 & GAGCGTTGACCTGTGAGAGTCTC \\
\hline
\end{tabular}

${ }^{a}$ Numbers indicate location of primers with respect to the translational start site of the target gene. Negative numbers represent sites 5' of the translational start site, positive numbers represent sites $3^{\prime}$ of the translational start site. 


\subsection{Promoter Cloning and Construction of Promoter-Reporter Plasmids}

The pGreen/pSoup binary plant vector system was employed for the construction of promoter-reporter constructs in A. thaliana. The pGCO:GUS vector (Suárez-López, 1998; obtained from the Dr. Hepworth Lab, Carleton University) employed is derived from pGreen, a binary Ti vector for Agrobacterium-mediated plant transformation, that requires co-transformation into Agrobacterium with the pSoup vector. The pSoup vector provides the replication functions in trans to the pGreen vector, or the pGCO:GUS vector derived from it, in Agrobacterium (Hellens et al., 2000). This has allowed the size of the pGreen vector to remain small, at only $6.5 \mathrm{~kb}$. This pGreen-derived pGCO:GUS vector contains the E. coli uidA gene, which encodes the enzyme $\beta$-glucuronidase (GUS), fused to the Constans $(\mathrm{CO})$ promoter. When fused to a promoter, GUS acts as a reporter gene for investigating the spatial and temporal expression patterns of a given promoter. The selectable markers of the pGreen and pSoup vectors are the nptII and tet genes, respectively, which confer resistance to kanamycin and tetracycline, respectively.

Nested primers were designed to amplify the promoter sequences of LAST, PS1 and PS2 from $B$. juncea gDNA. The resulting amplifications included the start codon of the respective genes. The inner primers of each nested pair contained the restriction sites NcoI ( $\left.\mathrm{CCA}^{\wedge} \mathrm{TGG}\right)$ and $\mathrm{BamHI}\left(\mathrm{GGA}^{\wedge} \mathrm{TCC}\right)$, at the 5' and $3^{\prime}$ ends, respectively, for directional insertion immediately 5 ' of the GUS gene in the pGCO:GUS vector (Fig. 4, Table 5). These restriction sites remove the CO promoter from the pGCO:GUS vector and were not present in the promoters of interest. The two nested reactions comprised: Advantage 2 PCR buffer (Clontech), $0.2 \mathrm{mM}$ dNTPs, $1 \mathrm{mM} \mathrm{MgCl} 2$ (additional to buffer), $0.2 \mu \mathrm{M}$ each of the 3' and 5' oligonucleotide primers and Advantage 2 Polymerase mix 
(Clontech). The primary PCR contained $\sim 100 \mathrm{ng}$ of $B$. juncea gDNA, while the secondary reaction contained $2 \mu \mathrm{L}$ of a $1 / 50$ dilution of the primary PCR products. The thermal cycling was as follows: 10 min denaturation at $94^{\circ} \mathrm{C} ; 35$ cycles of 1 min denaturation at $94^{\circ} \mathrm{C}, 1 \mathrm{~min}$ annealing at $58-60^{\circ} \mathrm{C}$ (depending on primer $\mathrm{T}_{\mathrm{m}}$ values), 4-6 min extension at $72^{\circ} \mathrm{C}$ (depending on length of the target amplicons); followed by a final extension period of $10 \mathrm{~min}$ at $72^{\circ} \mathrm{C}$. The amplification products were gel purified (GFX kit, GE Healthcare) and ligated to the pGEM T-Easy vector. The LAST promoter truncations were constructed using the truncation primers, listed in Table 3, and the fulllength LAST promoter in pGEM as template.

The pGEM plasmids, containing PS1, PS2, LAST and LAST truncation sequences, and the pGCO:GUS plasmid were digested with $N c o I$ and BamHI (Fig. 4). Reactions were incubated at $37^{\circ} \mathrm{C}$ for 2.5 hours with 10 units of enzyme per $1 \mu \mathrm{g}$ DNA. The digested pCO:GUS vector was subsequently treated with 10 units of Antarctic phosphatase for $2 \mathrm{hr}$, to prevent self ligation by removal of the 5' phosphate groups (New England BioLabs). The inserts and the linearized, phosphatase-treated vectors were gel purified (GFX kit, GE Healthcare) prior to ligation. Ligation reactions comprised 100$200 \mathrm{ng}$ of total DNA, in a 3:1 insert:vector molar ratio, with 400 units of T4 DNA ligase in the supplied buffer (New England BioLabs) in a volume of $25 \mu \mathrm{L}$. The reactions were incubated at room temperature (RT) for 3 hours or at $10^{\circ} \mathrm{C}$ overnight prior to transformation of the E. coli strain DH10B. Transformants were screened on plates containing $50 \mathrm{ng} / \mathrm{mg}$ kanamycin, the selectable marker of the pGreen vector. Proper insertion of DNA was confirmed via screening by colony PCR with the same primers used for the second round of nested amplification of the target sequences from gDNA 
(Table 3). Plasmid was isolated with the Wizard Plus Miniprep kit (Promega) and sequenced over the insertion site using a primer designed within the GUS coding region (5' - CAG CAA TTG CCC GGC TTT CTT GTA). The isolated plasmid was used for transformation of Agrobacterium. 
Figure 4. Construction of promoter-reporter consructs in the modified pGreen vector. The digestion of the pGCO:GUS vector with BamHI and NcoI removes the Constans (CO) promoter and the promoter of interest is inserted in its place at the BamHI and NcoI sites. This example is for the LAST promoter sequence and the same procedure applies to all constructs. 

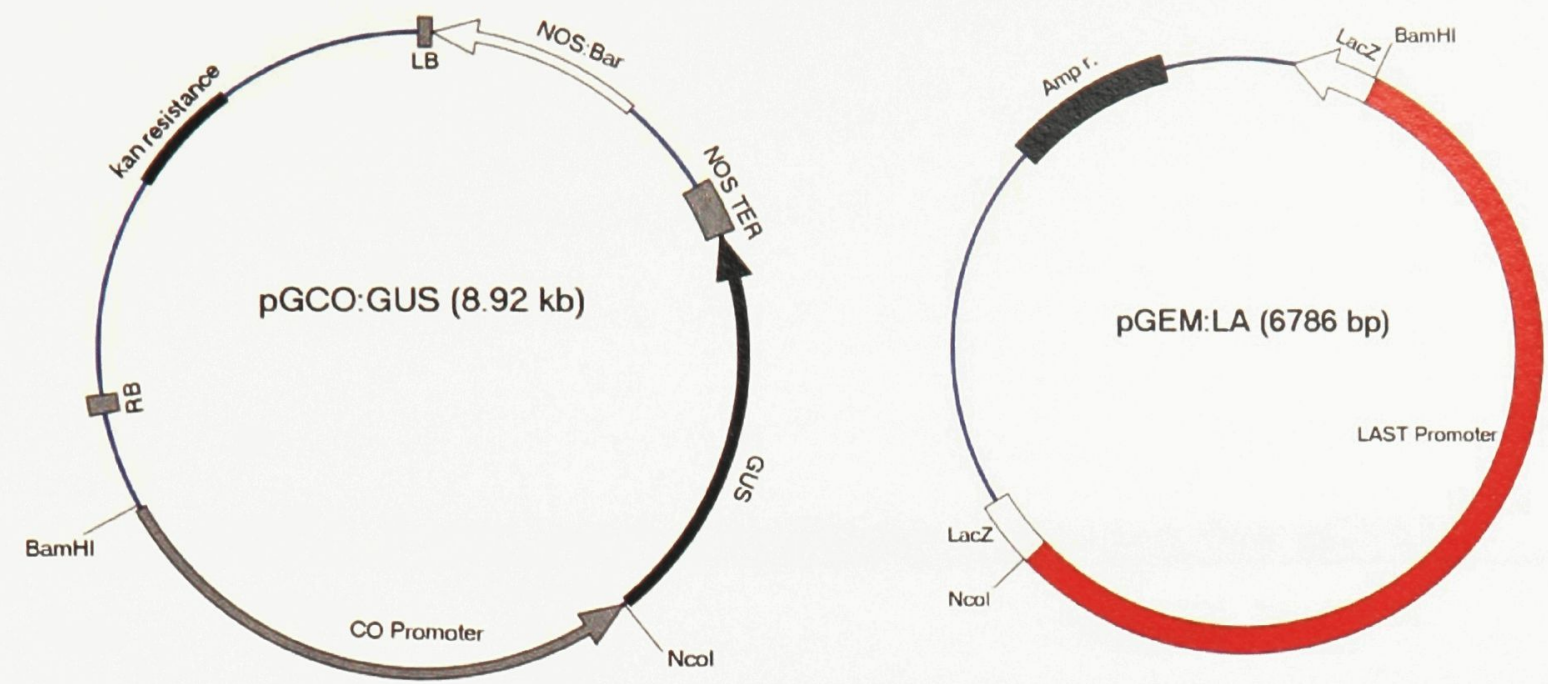

Digestion with $\mathrm{Ncol}$ and BamHI
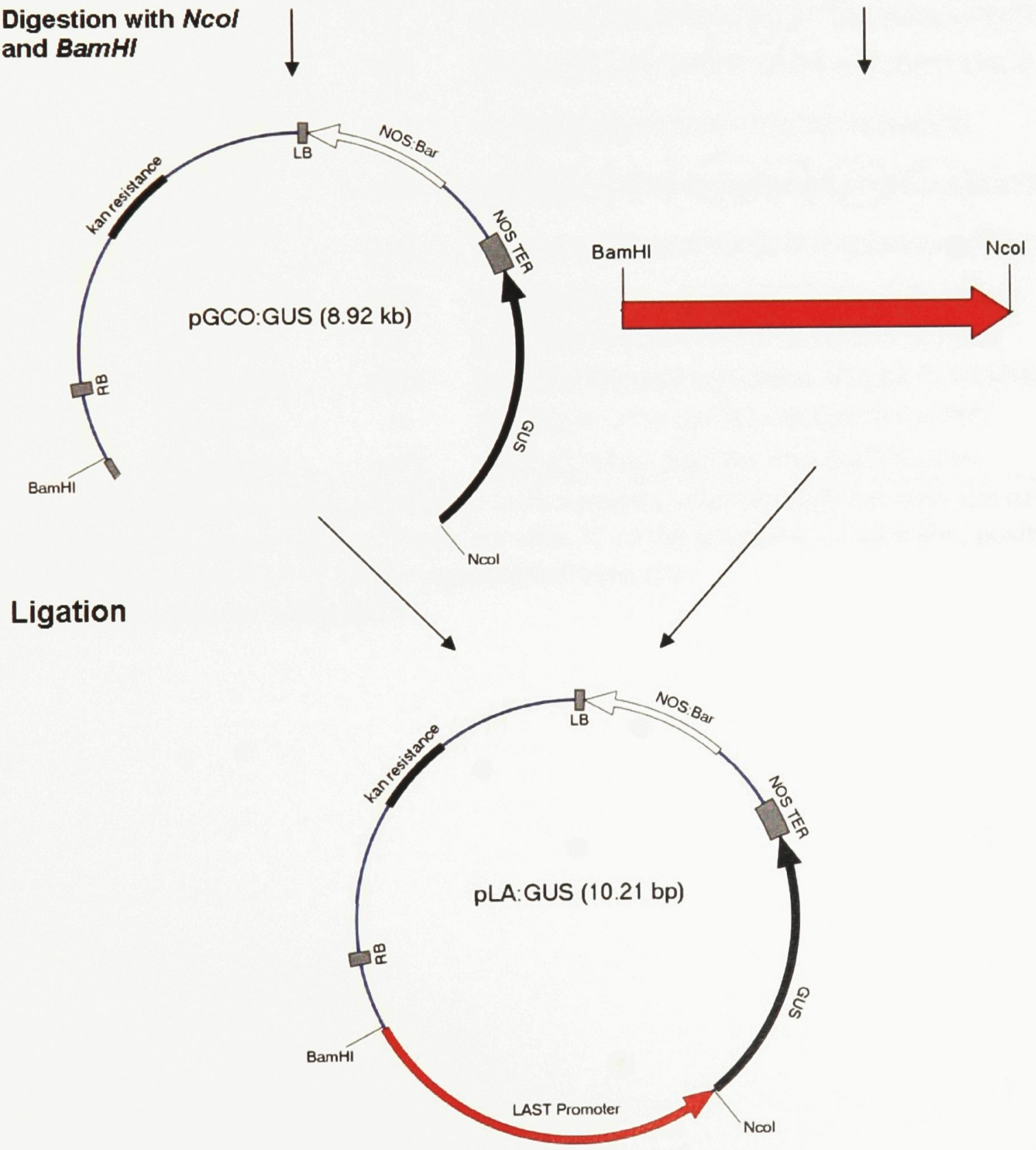
Table 5. Primers used for amplification of promoters from gDNA

\begin{tabular}{|c|c|c|c|}
\hline Gene & $\begin{array}{l}\text { Primer } \\
\text { Name }\end{array}$ & $\begin{array}{c}\text { Location } \\
\text { from } \\
\text { ATG }^{a} \\
\end{array}$ & Sequence $^{b}$ \\
\hline \multirow[t]{6}{*}{ LAST } & LA-f- $N$ coI & -9 & CATGCCATGGTCTGCAGCTCTATGCTCATTTTGTT \\
\hline & $\begin{array}{l}\text { LA-r-Tru1- } \\
\text { BamHI }\end{array}$ & -361 & CGGGATCCAACATTTCGTCAACTTGCTCCGCC \\
\hline & $\begin{array}{l}\text { LA-r-Tru2- } \\
\text { BamHI }\end{array}$ & -804 & CGGGATCCCTTTGTACTACACACGACCG \\
\hline & $\begin{array}{l}\text { LA-r-Tru3- } \\
\text { BamHI }\end{array}$ & -1485 & CGGGATCCAGCATCAGGGACATGAGAAACCGT \\
\hline & $\begin{array}{c}\text { LA-r-Tru4- } \\
\text { BamHI }\end{array}$ & -2148 & CGGGATCCTGATGGAGCAGTACGGGAGAGATT \\
\hline & LA-r-BamHI & -3724 & CGGGATCCGAAAGTGCTGGTGTAGTAGGACC \\
\hline \multirow[t]{2}{*}{ PS1 } & PS1-NcoI & -18 & CCATGGTAGCCATTACGACAGGTTTCTTGG \\
\hline & PS1-BamHI & -1452 & GGATCCTTCCGAAGCGGGAAGAAATATGAGTG \\
\hline \multirow[t]{2}{*}{ PS2 } & PS2-NcoI & -14 & CCATGGCCGTAGCCATTACCACAGGTTTC \\
\hline & PS2-BamHI & -1459 & GGATCCATAGTGGGGTAGAGTGTTGAAT \\
\hline
\end{tabular}

${ }^{a}$ Numbers indicate location of primers with respect to the translational start site of the target gene. Negative numbers represent sites 5' of the translational start site, positive numbers represent sites 3' of the translational start site.

${ }^{b}$ Restriction sites are underlined 


\subsection{Construction of LAST Promoter:gene Construct in pBAR1}

The coding sequence of the $B$. juncea LAST gene was amplified from root cDNA made from RNA of plants grown for 13 days after germination under conditions of sulfur deficiency. The root samples ( 100 mg) were frozen on dry ice, ground quickly with a Teflon Eppendorf pestle in $1.5 \mathrm{~mL}$ microcentrifuge tube, prior to addition of lysis buffer (RLT, Qiagen RNeasy Mini kit), and ground for a further five minutes with a tissue homogenizer ( $f=30 / \mathrm{s}$ ) (Retsch, model MM301 mixer mill). RNA was extracted using the Qiagen RNeasy Plant Mini kit and was quantified by absorbance spectroscopy at 260 nm. cDNA was synthesized from RNA by reverse transcriptase using an 18-nucleotide oligo-dT primer. RNA $(1 \mu \mathrm{g})$ and oligo-dT primer $(4.17 \mu \mathrm{M})$ in a volume of $12 \mu \mathrm{L}$ were incubated at $70^{\circ} \mathrm{C}$ for $5 \mathrm{~min}$, followed by chilling on ice for $2 \mathrm{~min}$. Subsequently, 20 units of RNase-inhibitor (Ambion), reverse transcriptase buffer (New England BioLabs) and dNTPs (final concentration $0.2 \mathrm{mM}$ ) were added and the mixture was incubated at $37^{\circ} \mathrm{C}$ for 5 minutes, prior to addition of 0.2 units of M-MuLV reverse transcriptase (New England BioLabs), and incubation at $42^{\circ} \mathrm{C}$ for $60 \mathrm{~min}$. Reverse transcriptase was inactivated by 10 min incubation at $70^{\circ} \mathrm{C}$ and the cDNA was stored at $-20^{\circ} \mathrm{C}$.

The $B$. juncea LAST coding sequence was amplified as 5' and 3' fragments, overlapping by $\sim 60 \mathrm{bp}$. The 5' fragment was amplified in a semi-nested PCR using the primers LA-cDNA1 and LA-cDNA4 in the primary reaction, followed by LA-r-pBAR1SacI and LA-cDNA4 in the secondary reaction (Table 6). Similarly, the 3 ' fragment was amplified by a semi-nested PCR with the primers LA-cDNA2 and LA-cDNA3 followed by PCR with the primers LA-cDNA3 and LA-f-pBAR1-BamHI (Table 6). The 5' and 3' LAST cDNA fragments were gel excised and purified using the GFX kit (GE Healthcare) 
and used as template ( 100 ng of each fragment) for overlap-extension PCR (Higuchi, 1990) using the LA-r-pBAR1-SacI (located at the translational start site of the LAST gene) and LA-r-pBAR1-BamHI (located at the TGA stop codon) primers to amplify the full length gene (Table 6). The resulting full-length amplicon was ligated to pGEM and sequenced.

The LAST promoter was amplified from the NcoI/BamHI-tagged LAST-pGEM construct with primers LA-r-BamHI and LA-f-BamHI, both containing BamHI restriction sites, and inserted into pGEM (Table 6). The 3' end primer contained the translationl start site of the LAST gene. 
Table 6. Primers designed for amplification of LAST coding region from cDNA

\begin{tabular}{|c|c|c|}
\hline Primer Name & $\begin{array}{c}\text { Location } \\
\text { from } \mathrm{ATG}^{a}\end{array}$ & Sequence $^{b}$ \\
\hline LA-r-BamHI & -3724 & CGGGATCCGAAAGTGCTGGTGTAGTAGGACC \\
\hline $\begin{array}{l}\text { LA-r-pBAR1 } \\
\text { BamHI }\end{array}$ & -33 & GGATCCCATTTTGTTATCTTTGTGGGTGTTGAAGTGGGT \\
\hline LA-cDNA2 & -18 & GTTCTCTATCАСССАСТTСААСАCC \\
\hline LA-f- $\mathrm{N}$ CoI & -9 & CATGCCATGGTCTGCAGCTCTATGCTCATTTTGTT \\
\hline $\begin{array}{l}\text { LA-f-pBAR1 } \\
\text { BamHI }\end{array}$ & 0 & CGGGATCCATGAGCATAGAGCTGCAGAGCC \\
\hline LA-cDNA4 & +1455 & CCAGGGTTTAACCGACCTTCG \\
\hline LA-cDNA3 & +1475 & CGACTTGACCGAGATGTTGACC \\
\hline $\begin{array}{l}\text { LA-f-pBAR1 } \\
\text { Sacl }\end{array}$ & +2446 & GAGCTCTCAACACATATCGTGCAACGTAGTTC \\
\hline LA-cDNA1 & +2479 & AACGCTGTCTTAACATACTTGTGC \\
\hline
\end{tabular}

${ }^{a}$ Numbers indicate location of primers with respect to the translational start site of the target gene. Negative numbers represent sites 5' of the translational start site, positive numbers represent sites 3' of the translational start site.

${ }^{b}$ Restriction sites are underlined 
The LAST coding sequence in pGEM was directionally inserted at the SacI and BamHI sites of the pBAR1 plasmid. A nos terminator site exists downstream of the LAST gene to ensure termination of translation. The SacI (NEBuffer 1) and BamHI (NEBuffer 3) restriction endonucleases require different buffer conditions. Therefore, a sequential digest was conducted on the LAST:pGEM vector and pBAR1. The pBAR1 vector was phosphatased, as described previously, following digestion. Transformants, selected on LB plates containing $50 \mu \mathrm{g} / \mathrm{mL}$ kanamycin monosulfate, were screened by colony PCR. The BamHI-tagged LAST promoter in pGEM was digested with BamHI and inserted into the BamHI site of the pBAR1 plasmid containing the LAST coding region. Successful transformants were screened for proper orientation using the LA-Tru1 and the LA-cDNA4 primers (Tables 5 and 6) located in the promoter and coding sequences, respectively. The resulting LAST:pBAR1 construct was transformed into Agrobacterium.

\subsection{Preparation of Electrocompetent Agrobacterium}

A 10-mL culture of Agrobacterium tumefaciens strain C58C GV3101 pMP90 RK, started from a freezer stock, was incubated in $\mathrm{LB}$ media at $200 \mathrm{rpm}$ at $28^{\circ} \mathrm{C}$ for $2-3$ days and used to inoculate $1 \mathrm{~L}$ of LB media, which was similarly incubated until an $\mathrm{OD}_{600}$ of 0.5 was reached. The culture was chilled on ice for $15 \mathrm{~min}$ and centrifuged at $8000 \mathrm{rpm}$ for $10 \mathrm{~min}$ at $4^{\circ} \mathrm{C}$. The cells were washed in successively smaller volumes of sterile Milli-Q $\mathrm{H}_{2} \mathrm{O}$ (Millipore corp.), $1 \mathrm{~L}, 500 \mathrm{~mL}$, and $50 \mathrm{~mL}$, to reduce the ionic content and concentrate the cells. The cells were then washed in $50 \mathrm{~mL}$ of ice-cold $10 \%$ glycerol, resuspended in $5 \mathrm{~mL}$ of ice-cold $10 \%$ glycerol and stored at $-80^{\circ} \mathrm{C}$ in $40-\mu \mathrm{L}$ aliquots. 


\subsection{Agrobacterium Transformation}

Transformation of Agrobacterium with the LAST:pBAR1 and pGreen/pSoup promoter:reporter constructs was performed in preparation for floral dip of A. thaliana plants. Two 40- $\mu \mathrm{L}$ aliquots of electrocompetent Agrobacteria were thawed on ice, 30$100 \mathrm{ng}$ plasmid (100 ng of each plasmid for cotransformation of pGreen and pSOUP) was added and the mixture was placed in a pre-chilled electroporation cuvette with a $0.2-\mathrm{cm}$ gap (Biorad). The electroporation conditions were $2.5 \mathrm{kV}, 25 \mu \mathrm{F}$ and $400 \Omega$ for $9 \mathrm{~ms}$. LB media $(1 \mathrm{~mL})$ was immediately added to the cuvette and the mixture was transferred to a microcentrifuge tube and incubated at $200 \mathrm{rpm}$ and $28^{\circ} \mathrm{C}$ for $3 \mathrm{~h}$. Cells were subsequently plated on LB plates containing $1.5 \%$ agar, $50 \mu \mathrm{g} / \mathrm{mL}$ kanamycin monosulfate (Fisher Scientific), $100 \mu \mathrm{g} / \mathrm{mL}$ rifampicin (Sigma), $40 \mu \mathrm{g} / \mathrm{mL}$ gentamycin sulphate (Sigma) and $10 \mu \mathrm{g} / \mathrm{mL}$ tetracycline (when using pSOUP) (Sigma). The pGreen and pBAR1 plasmids contain a kanamycin resistance gene, while pSOUP provides tetracycline resistance, and the Agrobacterium strain is rifampicin and genamycin resistant. Freezer stocks (25\% glycerol) of successful transformants, as determined by colony PCR, were made for storage and subsequent use in A. thaliana floral dip.

\subsection{Floral Dip of Arabidopsis thaliana}

Arabidopsis thaliana (Col-0 wild type) seeds were vernalized at $4^{\circ} \mathrm{C}$ in the dark for 3 days on $0.8 \%$ agar containing AT nutrient solution (Table 2). The seeds were transferred to $22-{ }^{\circ} \mathrm{C}$ growth chambers, with 24 hour illumination with a light intensity of $90-160 \mu \mathrm{mol} /\left(\mathrm{m}^{2} \mathrm{~s}\right)$, and transplanted to 4 " pots of pro-mix soil after 2 weeks at a density of 7-8 plants per pot. The soil was fertilized prior to planting with 20-20-20 all purpose 
fertilization mix (Plant-Prod, Brampton ON). Plants were grown in pots for 6 weeks, with the primary influorescence shoots removed during the fifth week to encourage more secondary shoots. A pair of 10-pot trays was used for each transformation.

Two 500-mL cultures of LB media, containing $50 \mu \mathrm{g} / \mathrm{mL}$ kanamycin, $100 \mu \mathrm{g} / \mathrm{mL}$ rifampicin, $40 \mu \mathrm{g} / \mathrm{mL}$ gentamycin sulphate and $10 \mu \mathrm{g} / \mathrm{mL}$ tetracycline (when using the pGreen-derived/pSOUP expression system) were inoculated with a pair of $10-\mathrm{mL}$ cultures of Agrobacterium, containing the appropriate plasmids (pBAR1 or pGreen/pSoup). The 500-mL cultures were incubated at $200 \mathrm{rpm}$ and $28^{\circ} \mathrm{C}$ until the $\mathrm{OD}_{600}$ reached 0.8 (approximately 20 hours). The cells were harvested by centrifuging at $7000 \mathrm{rpm}$ for $10 \mathrm{~min}$ and re-suspended in $500 \mathrm{~mL}$ of $5 \%$ sucrose. Silwet L-77 (Lehle Seeds, Round Rock TX) was added to each suspension to a final concentration of $0.02 \%$ v/v and mixed thoroughly. Each pot, containing 12 T0 plants with inflorescences between $2-10 \mathrm{~cm}$ in height, was inverted and dipped, with swirling, into the solution for 10 sec. The plants were subsequently placed on their sides and covered with plastic overnight before placing them in the growth chamber under a plastic hood to maintain high humidity. After flowering and silique development, approximately 2.5 weeks following floral dip, paper bags were placed over the inflorescences to retain all seeds as the pods dried. Plants were allowed to senesce for a further 3 weeks prior to collecting mature, dry seeds from each plant by rubbing the paper bags to break open the seed pods and sieving to remove debris. 


\subsection{Basta Selection of A. Thaliana Transformants}

The pGreen-derived and pBAR1 vectors contain the Bar gene conferring resistance to the microbially-produced phytotoxin bialaphos, also known as DLphosphinothricin (PPT), which is the active ingredient in the herbide Basta. The T1 seeds from the A. thaliana T0 plants transformed with pGreen-derived or pBAR1 plasmids were seeded at a density of approximately 10,000 seeds per tray and starting at 7 days after vernalization plants were sprayed with approximately $50 \mathrm{~mL}$ Basta $(250 \mathrm{mg} / \mathrm{L}$, also containing $0.01 \%$ v/v Silwet-L77 [Lehle Seeds, Round Rock TX]) per tray at $\sim$-day intervals to select for transgenic plants.

The selected T1 transgenic plants were self-pollinated and T2 generation seeds were collected and grown on AT plates (Table 2) containing $0.8 \%$ agar and $10 \mu \mathrm{g} / \mathrm{mL}$ PPT (Duchefa Biochemie, Haarlem, The Netherlands). In the T2 generation, a single insertion is expected to provide a 3:1 survival:death ratio during screening on PPTcontaining plates. To determine which $\mathrm{T} 2$ plants were homozygous, the $\mathrm{T} 2$ plants were self-pollinated to provide T3 seeds. The T3 seeds were tested for homozygousity on selection plates with $100 \%$ viable seed indicating a homozygous T2 plant. The T3 homozygous plants were used for GUS staining.

\subsection{GUS Staining}

Four to five A. thaliana plants of each line selected for GUS staining were grown on $0.8 \%$ agar in 2.5 " Magenta boxes containing $100 \mathrm{~mL}$ of nutrient solution. Plant samples for GUS staining were placed in $90 \%$ ice chilled acetone for $15 \mathrm{~min}$, or ice chilled heptane for $5 \mathrm{~min}$. The plant tissue was air dried for $30 \mathrm{~s}-1 \mathrm{~min}$, followed by 
washing in pre-chilled staining buffer $\left(50 \mathrm{mM} \mathrm{NaH}_{2} \mathrm{PO}_{4}, 0.5 \mathrm{mM} \mathrm{KFe}(\mathrm{CN})_{6}, 0.1 \%\right.$ Triton $\mathrm{X}-100)$ in a tissue culture plate for 2 min. The staining buffer was removed and seedlings were covered in 1-2 $\mathrm{mL}$ of staining buffer containing $1 \mathrm{mM}$ 5-bromo-4-chloro-3-indoxylbeta-D-glucuronide (X-Gluc) (Gold Biotechnology, Inc., St Louis, MO). The plate was sealed with Parafilm and incubated in the dark at $37^{\circ} \mathrm{C}$ for $15 \mathrm{~h}$. The staining solution was removed and tissues were incubated overnight with $70 \%$ ethanol at room temperature to remove chlorophyll. The ethanol was replaced with fresh $70 \%$ ethanol prior to observing the staining under a light microscope (SteREO Discovery v.20, Carl Zeiss, Inc.). Pictures were taken with the AxioCamMRc 5 (Carl Zeiss, Inc.) microscope digital camera, using the AxioVision Release 4.7.1 software.

\subsection{Sulfate-Limited and Cd Treated Plants}

GUS expression in the pLA:GUS plants was tested in two experiments: absence of sulfur and presence of $\mathrm{Cd}$ in growth media. Plants were grown vertically on plates containing $0.8 \%$ agar with the appropriate nutrient solution. For the sulfate deprivation experiment, sulfate salts used in the AT media were replaced with corresponding chloride salts for the sulfur deprivation experiment. The Cd treated plants were grown on AT media containing $1,10,50$ and $100 \mu \mathrm{M} \mathrm{CdCl}_{2}$. GUS staining for each experiment was performed, as described above, after 3 and 4 weeks of growth for the sulfate deprived plants, and 2 and 3 weeks for the $\mathrm{Cd}$ treated plants. 


\section{Results}

\subsection{Genome Walking}

There is little sequence information available for $B$. juncea. Therefore, the putative promoter regions of the targeted genes of the phytochelatin biosynthetic pathway were identified by genome walking and sequencing. The existing Genbank sequence data, in the form of complete or partial cDNAs of the B. juncea LAST (AJ223495), ATPS (ATP sulfurylase, AJ223499), APSR (APS reductase, AJ001207), OASS (O-acetyl serine sulfurylase, Y10845), GCL ( $\gamma$-glutamyl cysteine ligase, X95563), GS (glutathione synthase, Y10984), GR (glutathione reductase, AF109694) and PS (phytochelatin synthase, AJ278627) genes, was employed as the starting point for genome walking. Each of these sequences have been reported in phytoremediation-related papers with respect to $\mathrm{Cd}$ exposure (Table 1). Nested, gene-specific primers were based on these sequences for the amplification of gDNA segments upstream of the translational start site, with the goal of obtaining $\sim 4000 \mathrm{bp}$ of sequence. This process required multiple genome-walking steps for each gene. Sequences of approximately 4000 bp were obtained by Colleen Woodhouse for LAST, GCL, GS and GR. Therefore, extending the known sequence of the upstream regions of the ATPS, OASS, APSR and PS genes was the initial objective of this study (Table 7). 
Table 7. Length of promoter sequences obtained by genome walking

\begin{tabular}{|lccc|}
\hline Gene & $\begin{array}{c}\text { Total length of } \\
\text { promoter sequence } \\
\text { (bp) }\end{array}$ & $\begin{array}{c}\text { Initial } \\
\text { sequence (bp) }\end{array}$ & $\begin{array}{c}\text { Sequence } \\
\text { added (bp) }\end{array}$ \\
\hline ATPS & 3924 & 1547 & 2377 \\
APSR & 425 & 425 & 0 \\
OASS & 951 & 951 & 0 \\
PS1 & 1752 & 1752 & 0 \\
PS2 & 1869 & 1069 & 800 \\
\hline
\end{tabular}

${ }^{a}$ Sequence obtained by Colleen Woodhouse. 


\subsubsection{Genome Walking of ATPS}

A 2377-bp segment of the ATPS promoter region was generated in two genome walking steps, bringing the total to 3924 bp of sequence 5' of the start codon of ATPS (Table 1). However, when the entire 5' segment was amplified from B. juncea gDNA, the sequence of this fragment did not align with the sequences obtained by genome walking. Therefore, a diagnostic PCR was performed using four promoter-specific primers in combination with two gene specific primers. The promoter specific primers were designed to amplify approximately $500,1000,2600$, and $3600 \mathrm{bp}$ of the sequence 5 ' of the translational start site, when used in combination with the gene-specific primers. The gene-specific primers were designed 50 and $250 \mathrm{bp} 3$ 'of the translational start site, in the coding region of the ATPS gene. Amplicons of the expected sizes were not observed, suggesting that the initial genome walking sequence amplified non-specific fragments. The initial genome walk overlapped the ATPS Genbank sequence (AJ223499) by 66 bp.

\subsubsection{OASS and APSR}

The OASS, APSR and PS promoters were recalcitrant to amplification following the initial round of genome walking. This may be due to the choice of restriction enzymes used to make the genome walker libraries. For example, if the restriction sites are more than $\sim 6000$ bp from the gene-specific primer sites amplification would not occur without increasing the extension time in the PCR amplification. Alternatively, if the restriction sites are too close (less than $\sim 300 \mathrm{bp}$ ) to the region of amplification, it would be difficult to obtain substantial new sequence. Therefore, to circumvent these challenges, additional genome walker libraries were created using two new restriction 
enzymes (PsiI (TTA^^AAA) and BstZ17I (GTA^TAC)). These enzymes are AT rich and produce blunt ends, similar to the original four enzymes chosen (EcoRV (GAT^ATC), DraI (TTT^AAA), HpaI (GTT^ACC) and Scal (AGT^ACT)). Two sets of nested genome walker primers for each gene were designed, but the OASS amplification products contained no new additional sequence. Therefore, the total sequence of the OASS region $5^{\prime}$ of the translation start site is 951 bp (Table 7). This sequence was obtained by Colleen Woodhouse from a combination of 2 successive genome walks, with the initial amplification comprising 33 bp of the OASS coding sequence.

Amplification was also not observed for the 2 sets of nested primers designed to continue genome walking of the APSR promoter region. Therefore, the total sequence for the APSR promoter is $425 \mathrm{bp}$, which was obtained by Colleen Woodhouse in a single genome walking amplification (Table 7), which comprised $33 \mathrm{bp}$ of the APSR coding sequence. This sequence was amplified, however, in 3 of the 6 libraries. Therefore, additional sequence would have to be amplified from the remaining 3 libraries. If the remaining libraries contained specific targets of greater than $6000 \mathrm{bp}$, then it is unlikely that additional sequence to the 425 bp would be obtained.

\subsubsection{PS}

The initial genome walking of the promoter region of the PS gene by Colleen Woodhouse provided two distinct sequences, extending into the 5 , noncoding region from the start codon of the PS gene. This suggests the presence of two PS isoforms with sufficient conservation of nucleotide sequence in their coding regions to allow the nested genome walking primers to anneal to both genes. This is possible since the primary set 
of genome-walking primers were designed from the coding region of the PS gene. The new PsiI and BstZ17I genome walker libraries and two additional sets of nested primers did not enable the addition of new sequence to the 1755 bp of the PS1 isoform obtained by Colleen Woodhouse (Table 7 and Fig. 5A). The 1755-bp, PS1 sequence was obtained in a single genome walk, from 2 libraries, and overlapped the coding sequence by 55 bp. An additional 800 bases of sequence was added for the second isoform (PS2), from a single genome walk (Fig. 5B) using primers designed based on the 5' end of the 1069-bp segment obtained by Colleen Woodhouse, which was obtained in a single genome walk from 2 distinct libraries and overlapped the PS coding sequence by $53 \mathrm{bp}$. Therefore, a total of $1869 \mathrm{bp}$ of sequence is available for the PS2 isoform (Table 7).

The PS1 and PS2 promoter regions were subsequently amplified from B. juncea gDNA, as $\sim 1500$-bp segments (Fig. 5C). The sequences of both fragments match those obtained from genome walking. The two amplified PS promoter segments were inserted into the pGCO:GUS vector, in place of the $\mathrm{CO}$ promoter, to enable expression analysis in A. thaliana. To confirm the existence of different PS isoforms in B. juncea, PCR fragments of the coding region of each PS isoform were obtained and sequenced, as differences in the coding sequence and in promoter/UTR regions would be diagnostic of distinct PS isoforms. A nested pair of primers within the coding region was designed by aligning known PS coding sequences from 7 species of the Brassicacea family (Fig. 5A, B). These primers were used in combination with primers designed $\sim 50 \mathrm{bp} 5$ ' of the start codon for each of the isoforms to amplify isoform-specific regions from $B$. juncea gDNA. The 50-bp region immediately 5' of the translation start site likely comprises the 5, UTR and is not conserved between the $B$. juncea PS1 and PS2 sequences. Fragments 
obtained were larger than the expected size, based on the $B$. juncea PS1 cDNA sequence (Genbank Accession: AJ278627), suggesting the presence of intron(s). Sequence analysis demonstrated differences between the fragments amplified with the PS1 and PS2-specific nested primers. The coding sequence of the PS1 amplicon is $100 \%$ identical in coding sequence to the $B$. juncea PS1 cDNA sequence in Genbank (Genbank Accession: AJ278627), while the PS2 fragment is 93\% similar. The 5' UTR of the PS1 mRNA in Genbank was $89 \%$ identical to the obtained PS1 sequence, while no similarity was observed for the PS2 segment corresponding to the 5' UTR. These diagnostic tests suggest the PS1 promoter sequence is that of the $B$. juncea PS1 gene, and that the 1869 bp of PS2 promoter, and 900 bp of genomic sequence in the coding region, correspond to an unreported isoform of PS in the B. juncea genome. Three and four introns were found in the PS1 and PS2 genes, respectively. 
Figure 5. Genome walking and cloning of B. juncea PS1 and PS2 promoters. (A, B) Schematics represent the combined sequences from genome walking, gDNA amplifications and sequence from Genbank for the (A) phytochelatin synthase 1 (PS1) and (B) PS2 isoforms. The primers and their direction are indicated by arrows. The specific location, sequence and name of primers are listed in Table 1 of the Appendix. The translational start and stop codons, ATG and TGA respectively, and the 5' and 3' UTR's were determined for PS1 from the $B$. juncea PS1 sequence in Genbank (AJ278627). (C) PS1 (lane 2) and PS2 (lane 5) promoter segments amplified from $B$. juncea gDNA with primers containing $\mathrm{NcoI}$ and BamHI restriction sites for insertion into pGCO:GUS (Table 5C). (D) Diagnostic PCR of PS1 and PS2 coding region segments using $B$. juncea gDNA as template. Expected sizes were calculated based on combined promoter and Genbank (AJ278627) sequences, which do not include introns (Table 5D). 
A

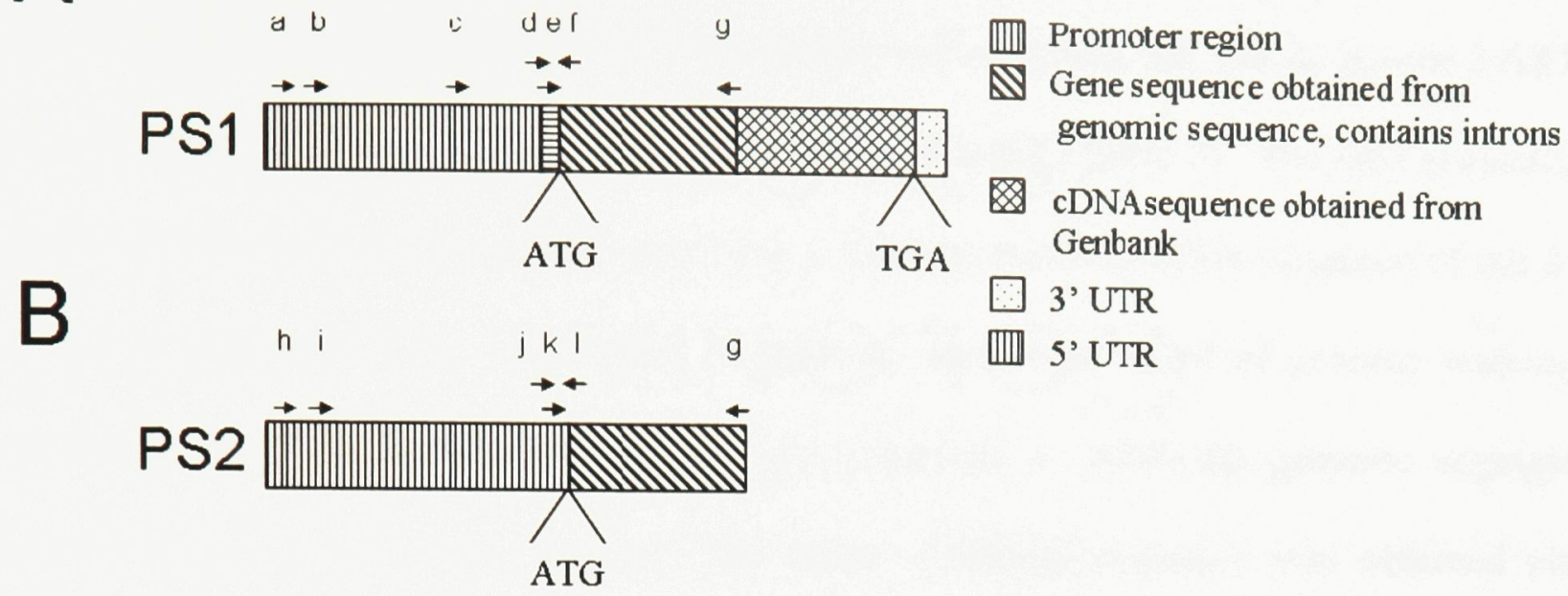

C

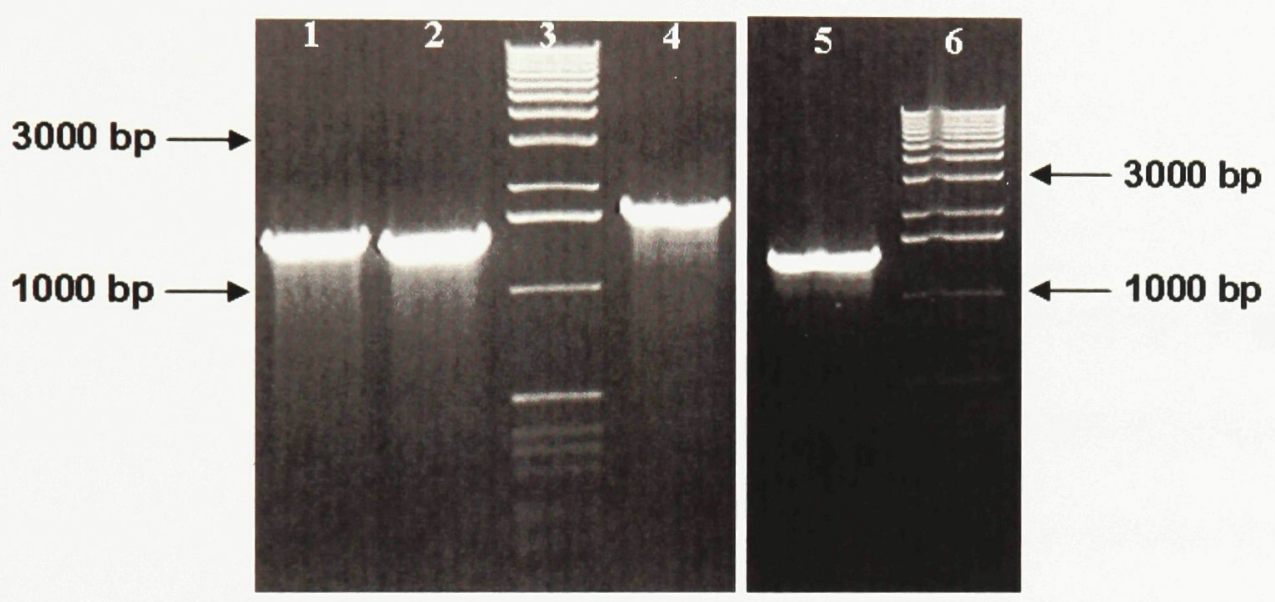

Table 5C. Primers used to amplify PS1 and PS2 promoters

\begin{tabular}{|cccc|}
\hline Lane & Gene & Primer Set & Expected Size (bp) \\
\hline 1 & PS1 & $\mathrm{a}+\mathrm{f}$ & 1587 \\
2 & PS1 & $\mathrm{b}+\mathrm{f}$ & 1485 \\
4 & PS2 & $\mathrm{h}+\mathrm{l}$ & 1852 \\
5 & PS2 & $\mathrm{j}+\mathrm{l}$ & 1491 \\
\hline
\end{tabular}
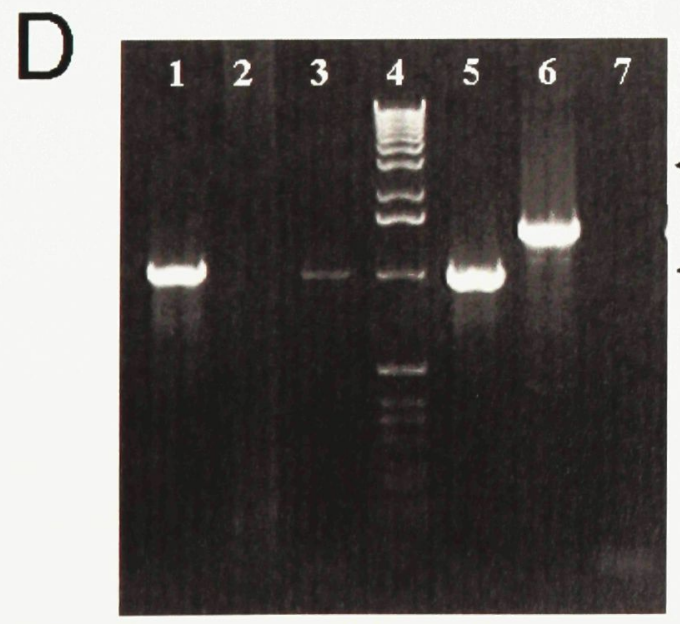

Table 5D. Primers used to amplify PS1 and PS2 coding sequences

\begin{tabular}{|c|c|c|c|c|}
\hline \multirow{7}{*}{$\longleftarrow 1000$ bp } & Lane & Gene & $\begin{array}{l}\text { Primer } \\
\text { Set }\end{array}$ & $\begin{array}{l}\text { Expected Size } \\
\text { (bp) }\end{array}$ \\
\hline & 1 & PS1 & $d+g$ & At least $550 \mathrm{bp}$ \\
\hline & 2 & PS2 & $j+g$ & At least $597 \mathrm{bp}$ \\
\hline & 3 & PS2 & $j+g$ & At least $597 \mathrm{bp}$ \\
\hline & 5 & PS2 & $e+g$ & At least 502 bp \\
\hline & 6 & PS1 & $c+g$ & At least $1135 \mathrm{bp}$ \\
\hline & 7 & PS2 & $k+g$ & At least $540 \mathrm{hp}$ \\
\hline
\end{tabular}




\subsubsection{LAST}

A 3771-bp fragment, 5' of the translational start site, for the B. juncea LAST gene was amplified and sequenced by Colleen Woodhouse (Table 7). The only sequence available for the $B$. juncea LAST gene was a 1163-bp partial mRNA sequence of the 3' end (Genbank accession: AJ223495). Therefore, the initial round of genome walking started at the $5^{\prime}$ end of this sequence and produced a $\sim 1300-$ bp genomic segment correspondng to the coding region. The entire $\sim 5000$-bp sequence was obtained via genome walking in 4 amplification cycles.

\subsubsection{Bioinformatic Analysis of LAST Promoter Region}

The 3771-bp segment corresponding to the promoter region of the LAST gene was analyzed for cis-regulatory elements, with the goal of identifying putative regulatory regions which could be investigated experimentally. The cis-regulatory elements in the promoter of the $B$. juncea LAST gene were identified and compared to those of the corresponding promoters of the two A. thaliana LAST genes (Sultr 2;1 and Sultr2;2), using the PLACE database (Higo et al., 1999), by honors-thesis student Brian Turnbull. Figure 6 illustrates selected environmental-response elements and transcription-factor binding sites found in the $B$. juncea promoter and absent or less prevalent in the corresponding $A$. thaliana promoter sequences. The $B$. juncea LAST gene that is the target of this study is most closely related to Sultr2;2 of A. thaliana. The promoter region of the $B$. juncea LAST gene contains 28 copper response elements (CuRE), GTAC, sites and four sulfur response elements (SuRE), GAGAC, sites. The corresponding Sultr2;1 and Sultr2;2 of the A. thaliana LAST promoters contain ten and 26 CuRE sites, 
respectively, and four and two SuRE sites, respectively. Interestingly, a 7-bp heavy metal response element (MRE-like, GCCTGCA), as determined by promoter:GUS experiments in tobacco protoplasts, was found by searching for this sequence in the $B$. juncea LAST promoter segment (Qi et al., 2007). In addition, an 11-bp portion (ATTCCAAAATTC) of a 40-bp sequence found to enhance transcription in the presence of metal ions (Qi et al., 2007) was found in the B. juncea LAST promoter. Detailed analysis of this novel MRE has not been completed and thus the specific regulatory region within the $40 \mathrm{bp}$ segment is unknown. A set of four truncation constructs of the LAST promoter were constructed by Brian Turnbull to allow identification of novel regulatory elements, and to test the effects of the putative CuRE, SuRE and MRE elements on transcription in the closely related, model species A. thaliana (Fig. 6). Of the 4 truncations designed, the Tru1, Tru2 and Tru3 sequences include or exclude the two MRE sites (Table 8). The fourth truncation, Tru4, was designed to break sequence between $1535 \mathrm{bp}$ and $3771 \mathrm{bp}$ upstream of the translational start site into two segments to test for the presence of regulatory elements. The promoter sequences were inserted into the pGCO:GUS vector, in place of the CO promoter, to drive the expression of the GUS reporter gene in A. thaliana. 


\section{Truncation Primers}

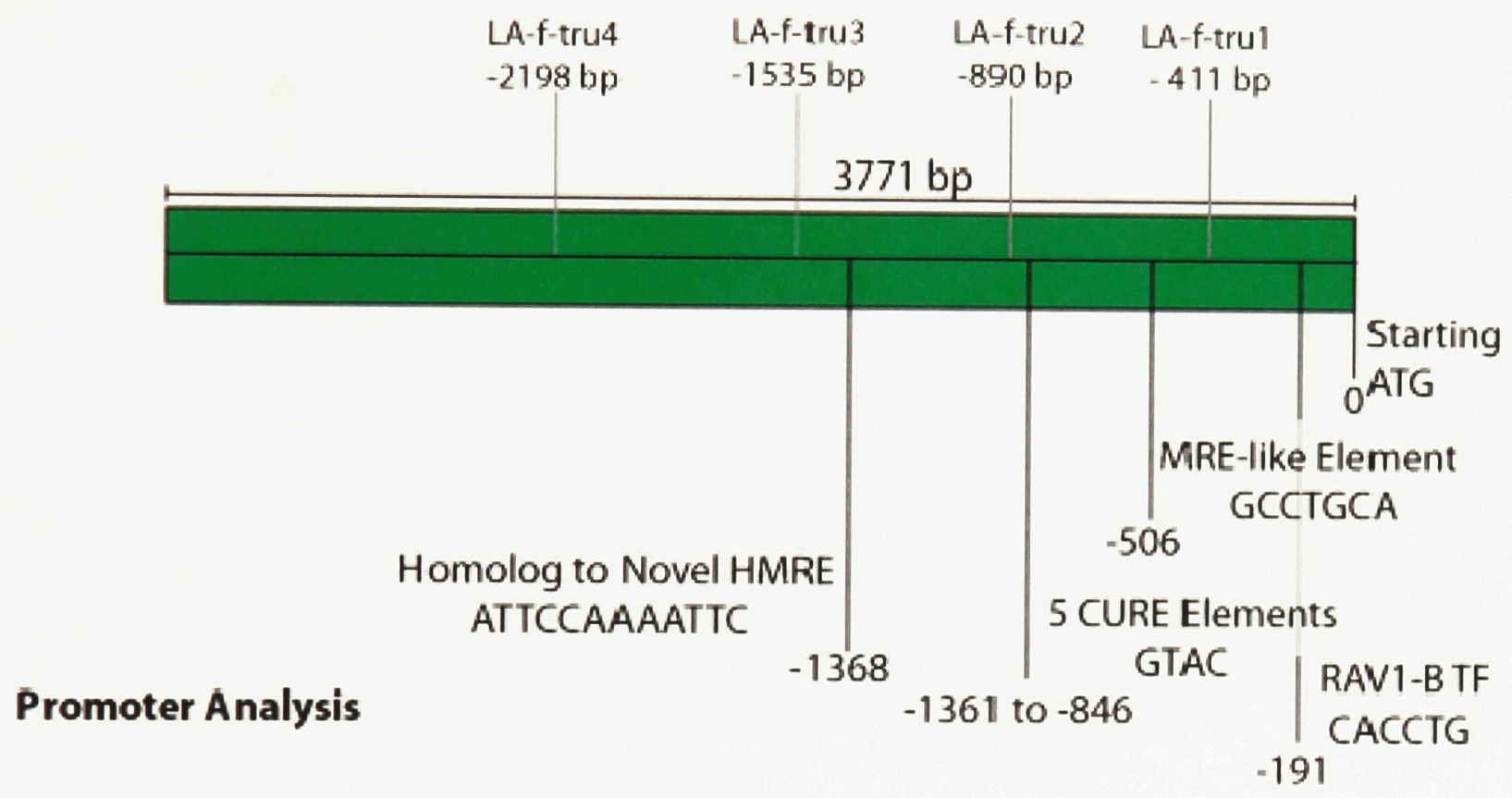

Figure 6. Selected results of bioinformatic promoter analysis and location of truncation primers for the B. juncea LAST promoter. Regulatory elements labeled represent putative metal response elements and the RAV1 transcription factor binding site (RAV1-BTF). Analysis was performed with the PLACE database (Higo et al., 1999). 
Table 8. Size of B. juncea low affinity sulfate transporter (LAST) promoter and truncations

\begin{tabular}{|ccc|}
\hline Promoter Fragment & Size (bp) & MRE Contained in Sequence $^{\boldsymbol{a}}$ \\
\hline LAST full-length & 3771 & Novel MRE and MRE-like \\
LAST Tru4 & 2198 & Novel MRE and MRE-Like \\
LAST Tru3 & 1535 & Novel MRE and MRE-Like \\
LAST Tru2 & 890 & MRE-Like \\
LAST Tru1 & 436 & None \\
\hline
\end{tabular}

${ }^{a}$ Novel metal regulatory element (MRE): ATTCCAAAATTC, MRE-Like: GCCTGCA (Qi et al., 2007) 


\subsection{Promoter:GUS Constructs}

The full-length LAST promoter and truncations 1-4 were constructed by Brian Turnbull in the pGCO:GUS vector. The CO promoter was replaced with the LAST promoter sequences, to drive the expression of the GUS reporter gene in A. thaliana. All promoter segments in the five reporter constructs included the start codon of the LAST gene in order to maintain appropriate spacing between the translational start site and regulatory elements. All constructs were verified for proper insertion of the promoter segments by diagnostic PCR. Amplified fragments covering the promoter-GUS junction were also sequenced to ensure the GUS gene start codon was in-frame with the start codon of the LAST gene at the 3' end of the promoter segments. The five pLA:GUS constructs were transformed into Agrobacterium and A. thaliana plants were subsequently transformed via floral dip. Similarly, 1481-bp and 1484-bp segments of the PS1 and PS2 promoter sequences, respectively, were inserted into the pGCO:GUS vector, replacing the $\mathrm{CO}$ promoter, and confirmed by PCR. The pPS1:GUS and pPS2:GUS constructs were transformed into Agrobacterium and A. thaliana plants were subsequently transformed, via floral dip, by Colleen Woodhouse. 


\subsubsection{A. thaliana Transgenic Plants}

Homozygous T3 A. thaliana plants of the LAST promoter-reporter constructs were used for GUS staining. Homozygous plants were obtained for 12 independent fulllength pLAST:GUS lines (Table 3), of which 6 out of 8 lines tested demonstrated GUS expression after 3 weeks of growth on $0.8 \%$ agar with $50 \%$ Hoaglands media. Homozygous T3 plants were obtained for 20, 19, and 10 independent lines of transformants from the Tru1, Tru3 and Tru4 constructs respectively (Table 9). Of these homozygous lines, 5 of 12 Tru1 lines tested showed some level of GUS staining. Consistent GUS staining was observed for 8 of 16 Tru3 homozygous lines. Similarly, 5 of 10 homozygous Tru4 lines displayed consistent GUS expression. 
Table 9. Number of transformant lines at each generation for pLA:GUS constructs

\begin{tabular}{|lccc|}
\hline \multicolumn{1}{|c}{ Construct } & $\begin{array}{c}\text { \# of T1 } \\
\text { Transformants }\end{array}$ & $\begin{array}{c}\text { \# T1 Lines } \\
\text { Properly } \\
\text { Segregated in T2 } \\
\text { Generation }^{a}\end{array}$ & $\begin{array}{c}\text { \# of Independent T2 } \\
\text { Homozygous Lines }^{b}\end{array}$ \\
\hline pLA:GUS & 18 & 16 & 12 \\
pLA-Tru4:GUS & 19 & 11 & 19 \\
pLA-Tru3:GUS & 26 & 22 & n/a \\
pLA-Tru2:GUS & 20 & 0 & 20 \\
pLA-Tru1:GUS & 34 & 23 & \\
\hline
\end{tabular}

${ }^{a}$ Properly segregated T1 lines did not deviate from a 3:1 survival:death ratio when T2 plants were grown on PPT media.

${ }^{5} \mathrm{~T} 2$ homozygous lines were determined by $100 \%$ survival rate of T3 plants grown on PPT media. 


\subsection{GUS Staining}

Plants used for preliminary screening of GUS expression were grown in magenta boxes with $0.8 \%$ agar and $50 \%$ Hoagland's media (Table 9) at a density of 5 plants per box. The transgenic A. thaliana plants expressing the pLA:GUS reporter constructs were analyzed for GUS expression after 3 weeks of growth, when the plant was in the 4-6 leaf stage and about to bolt, and after 4-5 weeks, when the plants had bolted and contained developing siliques. The full-length LAST promoter and the Tru3 (1535 bp) and Tru4 (2198 bp) constructs displayed similar GUS expression (Fig. 7). Expression was localized to new leaves developing at the shoot apex and present in the developing buds after bolting. GUS staining was also observed at the base of developing flowers, and in developing siliques of 4-5 week plants and was strongest in siliques which had yet to form distinct seeds (Fig. 7). No GUS staining was apparent in the seeds. Expression was also observed in cauline leaves, generally at the base and edge of the leaves. Two of the LAST:GUS lines displayed expression in other tissues, in addition to the pattern typical of the pLA, Tru3 and Tru4 lines. For example, the pLA:GUS B9 line, expressing with the full-length promoter, also showed expression in the roots and the stem. The root expression was predominately at secondary and tertiary root nodes, while the stem expression was strongest near the shoot apex. Another example is the B1 pLATru3:GUS line, which displayed additional expression in the vasculature of the rosette leaves. 


\section{A) PLA:GUS}
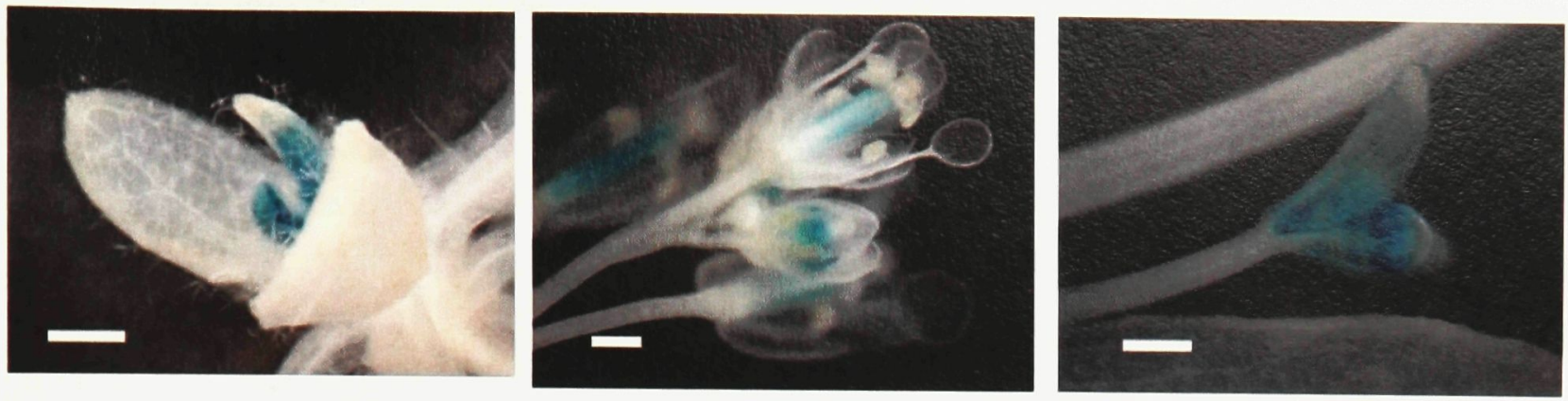

\section{B) pLA-Tru4:GUS}
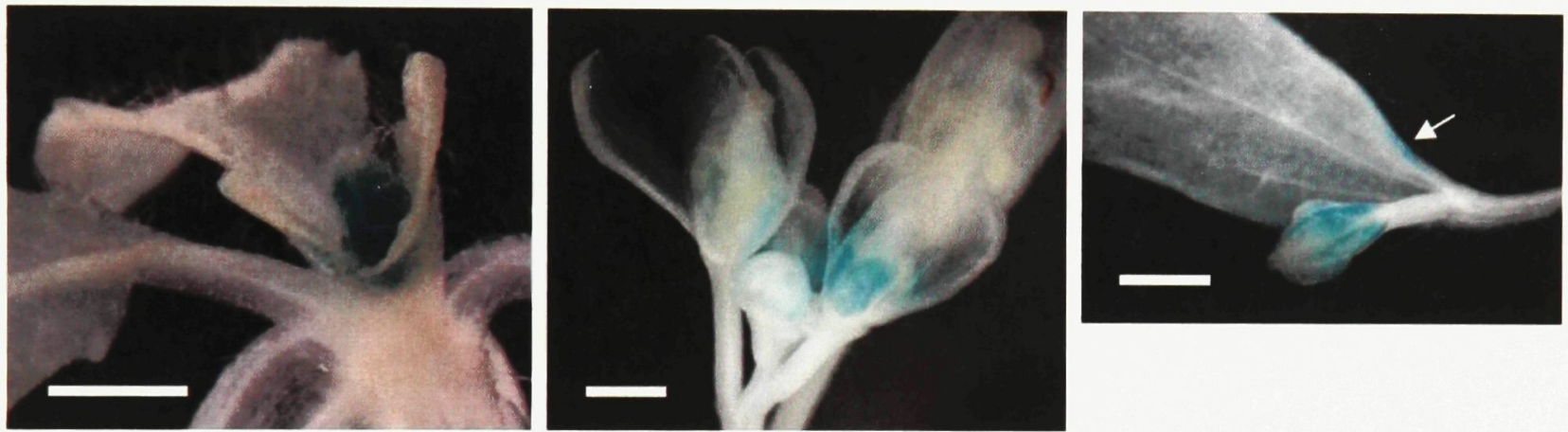

\section{C) pLA-Tru3:GUS}
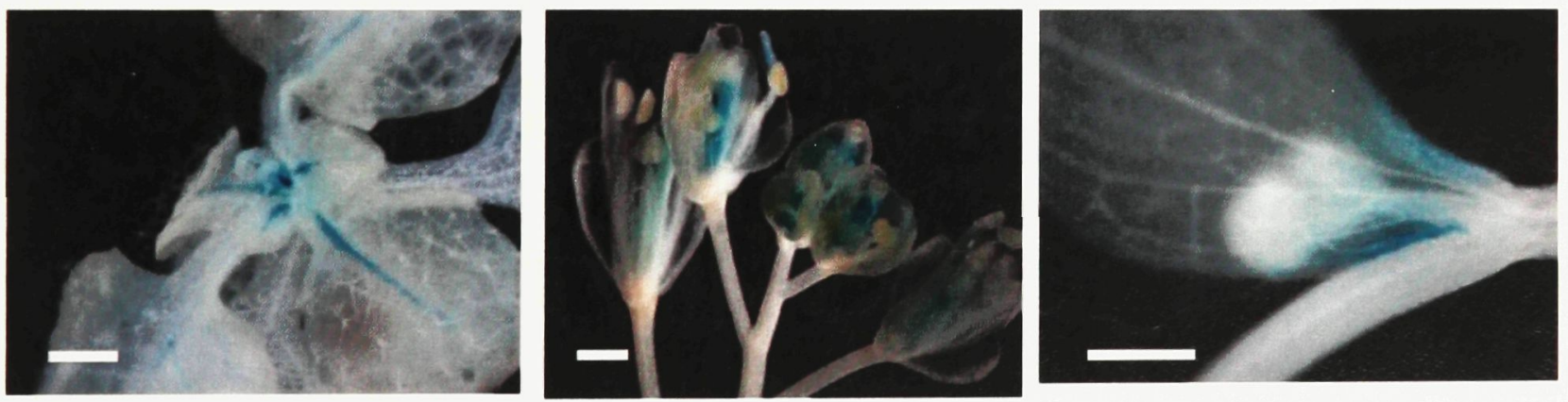

Figure 7. Representative GUS staining patterns of the (A) pLA:GUS, (B) pLATru4:GUS and (C) pLA-Tru3:GUS transgenic $A$. thaliana lines. Plants were grown for 3-5 weeks on $0.8 \%$ agar containing 50\% Hoagland's nutrient mixture. Representative lines are displayed for each construct. At least 5 homozygous T3 lines for each construct were tested. Scale bar is $500 \mu \mathrm{m}$. 
The pLA-Trul:GUS plants, which contain just 436 bp of LAST promoter sequence, displayed inconsistent expression of the reporter gene. Of the 12 lines tested, GUS staining was observed in only 5 and no common expression pattern was observed, as the staining varied from only in root to only in the trichomes of leaves. The variable expression of the reporter gene in these lines was likely due to positional effects. The 436-bp sequence may contain necessary elements to recruit basic transcription factors, thus acting as a minimal promoter. The Agrobacterium-mediated transformation is a random, rather than site-specific, method of inserting a target DNA sequence into the genome. Therefore, if the sequence is inserted downstream of regulatory elements, these elements could increase the transcription of the GUS gene, thereby resulting in the inconsistent expression pattern observed for the pLA-Tru 1:GUS construct.

\subsubsection{Sulfate Deprivation}

The pLA:GUS, pLATru3:GUS, and pLATru4:GUS plants were tested for the effect of sulfate deprivation on expression of the GUSreporter gene. Plants were germinated and grown vertically in Petri dishes on minimal AT (A. thaliana) media, and on minimal AT media in which sulfur salts were replaced with the corresponding chloride salt (Table 4). Approximately 5-6 plants were germinated per transgenic line per half dish, and 3 lines of each construct were tested. The plants showed no differences in size or flowering time when grown with or without sulfate in the growth media. Similarly, no difference was observed in GUS expression and the staining observed was the same as when plants were grown in 50\% Hoagland's (Fig. 7). 


\subsubsection{Cd Treatments}

Plants were grown vertically for 2 and 3 weeks on $0.8 \%$ agar containing AT media and $0,1,10,50$ or $100 \mu \mathrm{M} \mathrm{CdCl}_{2}$. A dose-dependent response was observed; plants decreased in shoot and root size with increasing $\mathrm{CdCl}_{2}$ concentration (Fig. 8). The plants treated with 50 and $100 \mu \mathrm{M} \mathrm{CdCl}_{2}$ were severely stressed, with necrotic and chlorosed leaves. GUS staining of the pLA:GUS, pLA-Tru3:GUS and pLA-Tru4:GUS plants showed no staining with 50 and $100 \mu \mathrm{M} \mathrm{CdCl}_{2}$ treatment. Staining of $1 \mu \mathrm{M} \mathrm{CdCl}_{2}$ plants was the same as the control for each construct. One of two pLA-Tru3:GUS lines and all three pLA-Tru4:GUS lines contained staining in the leaves of plants treated with $10 \mu \mathrm{M} \mathrm{CdCl}_{2}$ (Fig. 9). This staining was not observed in the three pLA:GUS lines tested. 


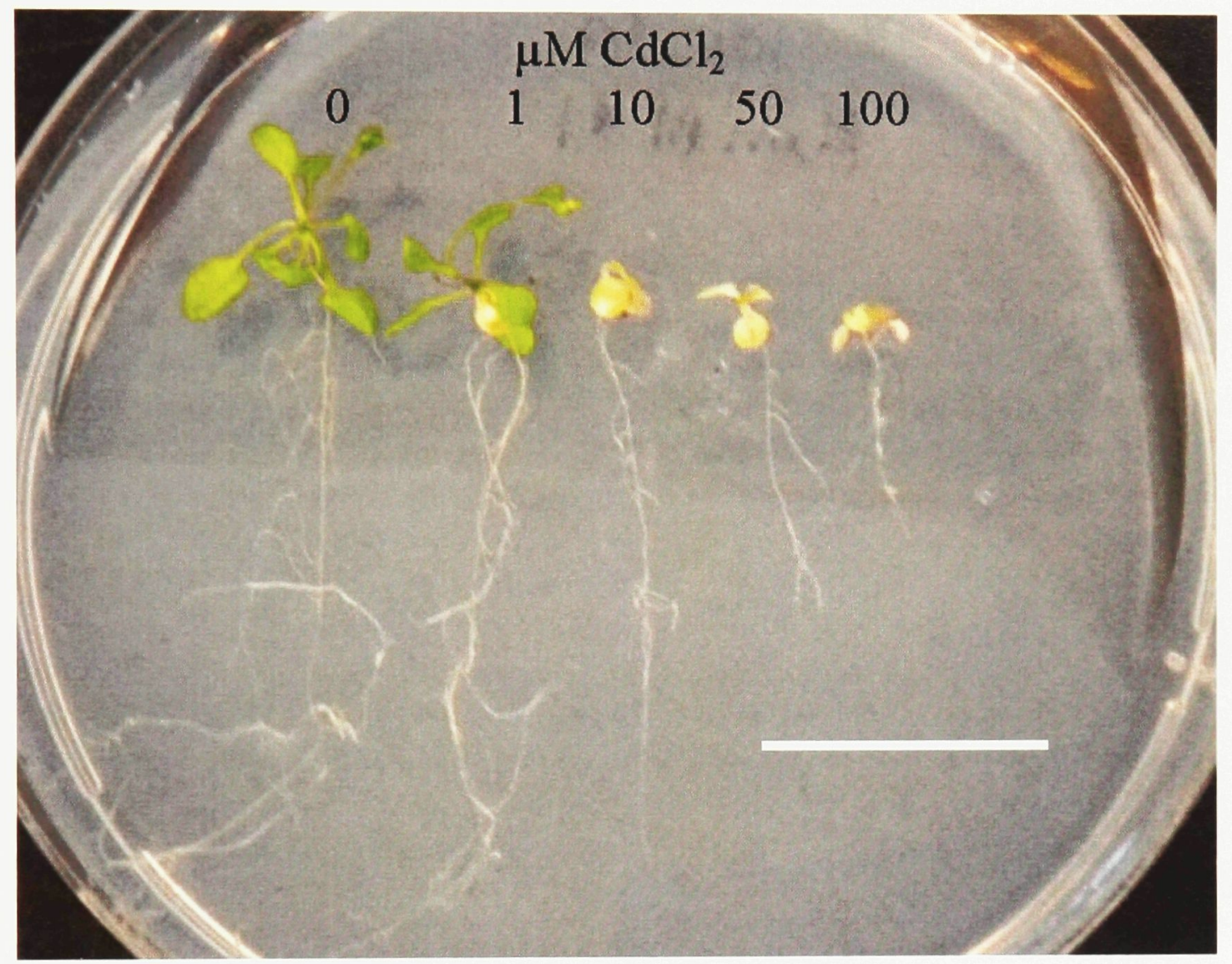

Figure 8. Effect of Cd treatment on wild-type A. thaliana. Plants were germinated and grown for 3 weeks on $0.8 \%$ agar containing AT media and $0,1,10,50$ or $100 \mu \mathrm{M}$ $\mathrm{CdCl}_{2}$. Scale bar is $2 \mathrm{~cm}$. 
Figure 9. GUS expression of the pLA-Tru4:GUS and pLA-Tru3:GUS transgenic $A$. thaliana lines with $10 \mu \mathbf{M ~ C d C l}_{2}$ treatment. Plants were grown for 2 or 3 weeks on $0.8 \%$ agar with AT media supplemented with $10 \mu \mathrm{M} \mathrm{CdCl}_{2}$. Three lines for each construct were tested. Scale bar is $500 \mu \mathrm{m}$. 
$0 \mu \mathrm{M} \mathrm{Cd}$

2 Weeks

A) PLA:GUS
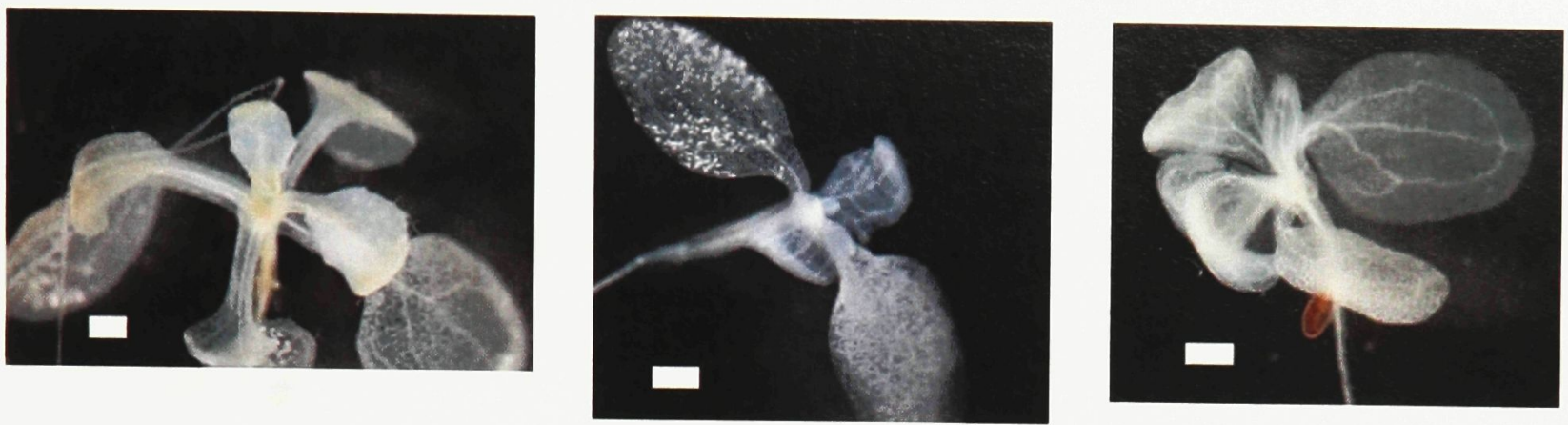

B) pLA-Tru4:GUS
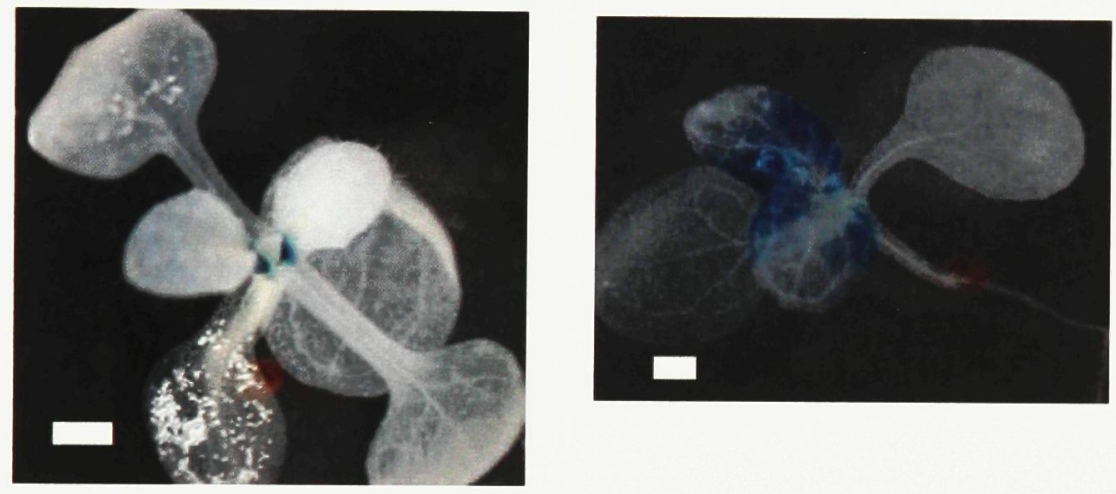

C) pLA-Tru3:GUS
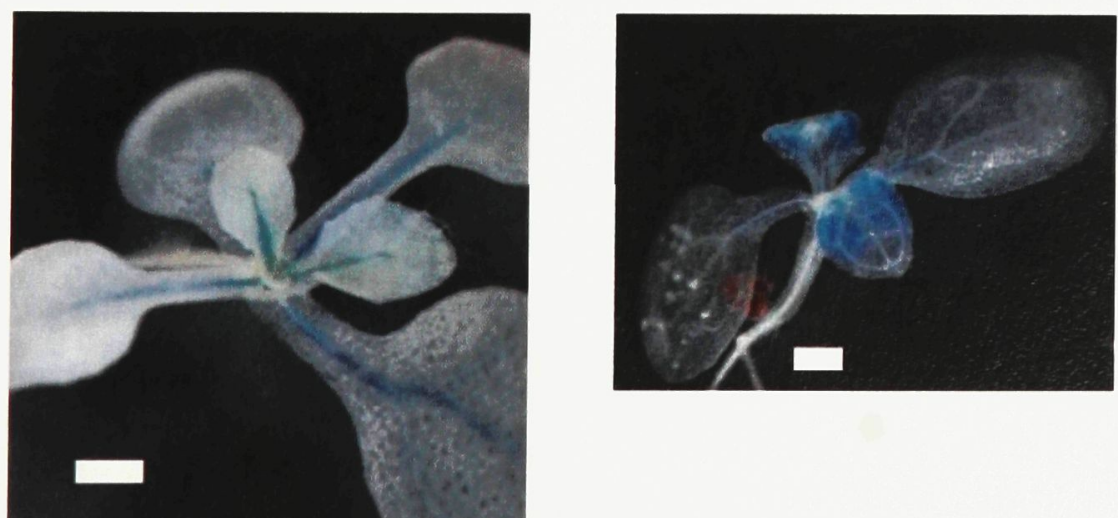

$10 \mu \mathrm{M} \mathrm{Cd}$ 3 Weeks 


\subsection{LAST:pBAR1 Construct}

In order to study the function of the $B$. juncea LAST gene, the coding sequence will be expressed in $A$. thaliana under control of the native B. juncea LAST promoter. A. thaliana plants transformed with this construct can be analyzed for differences in sulfur metabolism and heavy metal tolerance and accumulation. The LA:pBAR1 vector, containing the full-length promoter and coding region of the $B$. juncea LAST gene,was constructed in two steps. The LAST coding sequence was amplified from cDNA and inserted into the BamHI and SalI sites of pBAR1. The $4000-\mathrm{bp}, 5$ '-noncoding region, comprising the promoter was subsequently inserted immediately 5, of the coding sequence, at the BamHI site.

\subsubsection{LAST Gene Cloning}

The $B$. juncea LAST gene was amplified from cDNA of sulfate-deprived $B$. juncea roots. Primers were designed to amplify 5' and 3' segments ( $750 \mathrm{bp}$ each) of the gene. Since the $B$. juncea LAST mRNA sequence in Genbank (Accession: AJ223495) is a 1163-bp partial sequence of the 3' end of the cDNA, the genome walking sequence, obtained by Colleen Woodhouse was employed to design primers at the 5 ' end of the coding region (Fig. 10A,B). The full-length coding segment was subsequently assembled by overlap-extension PCR and ligated to pGEM (Fig. 10C). Primers used for the overlap-extension assembly step incorporated BamHI and Sall restriction tags at the 5, and 3 ' ends, respectively, of the amplified coding sequence to enable insertion at the corresponding sites of the pBARI vector. The coding sequence is identical at the amino 
acid level to the $B$. juncea mRNA partial sequence, and shares $92 \%$ identity to a sulfate transporter from B. oleracea (Genbank Accession: AM492198).

\subsubsection{LAST Promoter Cloning for pBARI}

The full-length LAST promoter segment was amplified from the pLA:GUS construct, with primers containing BamHI restriction sites, for insertion into the pBAR1 vector. The 3' primer also included the start codon of the LAST gene to ensure that there is no disruption of sequence or placement between the translational start site and any regulatory sequence, which could otherwise result from inserting a restriction site in the region immediately $5^{\prime}$ ' of the start codon. As a result, the LAST protein will be expressed with an amino-terminal, 3-amino-acid tag, resulting from the ATG of the promoter and the $6 \mathrm{bp}$ of the introduced BamHI restriction site. The LAST promoter segment, amplified from pLA:GUS, was introduced at the BamHI site of the pBARI construct containing the LAST coding sequence. The resulting LAST:pBARI construct was screened by PCR for proper orientation of the promoter segment. Sequencing over the promoter-gene junction demonstrated proper insertion of the promoter in frame with the start codon of the gene. 
Figure 10. Genome walking and cloning of the B. juncea LAST gene. (A) The schematic represents the promoter and coding region sequences combined from genome walking and the B. juncea partial cDNA sequence in Genbank (Accession: AJ223495). Primers, and their orientation, are indicated by the arrows. The specific location, sequence and name of primers are provided in Table 2 of the Appendix. The translational stop codon, labelled TGA, and the 3' UTR were obtained from the Genbank sequence. The putative translational start codon, ATG, was determined by alignment with the B. oleracea sulfate transporter sequence from Genbank (AM492198).

(B) Two overlapping fragments of the B. juncea LAST coding sequence were PCR amplified from cDNA made from RNA extracted from sulfate-deprived $B$. juncea roots. The 3' end of the gene was amplified in a nested PCR (lane 2, 4 and 5). Lane 2 shows the primary PCR fragment obtained using a primer outside of the coding region (Table 10B). Lanes 4 and 5 show the untagged PCR product and the 3' SalI tagged product, respectively, from the secondary reactions. The 5' fragment was reamplified via a secondary PCR reaction using the same primers as the primary reaction (Table 10B). The primary reaction contained no observable amplicon, and is not shown here. The 5' fragment was PCR amplified with no restriction tag and with a 5' BamHI tag (lanes 7 and 8, respectively) (Table 10B). (C) The 5' and 3' LAST coding region fragments were combined as template for overlap extension PCR. Lane 2 contains a PCR product amplified with primers containing no tags, while lane 2 shows a PCR product with SalI and BamHI tags for ligation into the pBAR1 vector (Table 10C). 
A

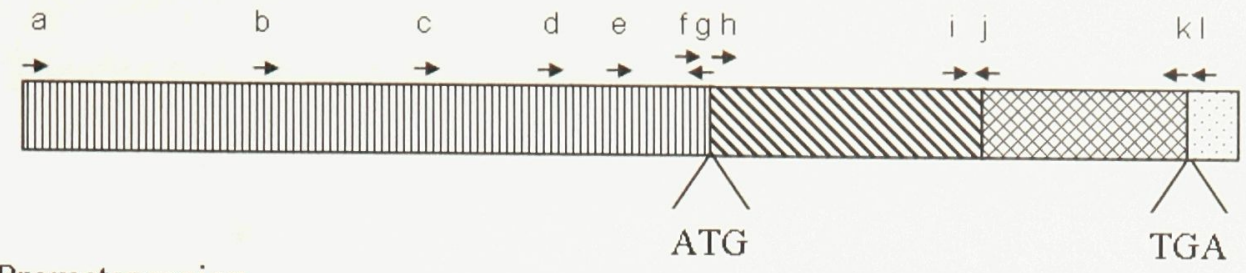

而 Promoter region

$\mathbb{N}$ Gene sequence obtained from genomic sequence, contains introns

cDNA sequence obtained from Genbank

3'UTR
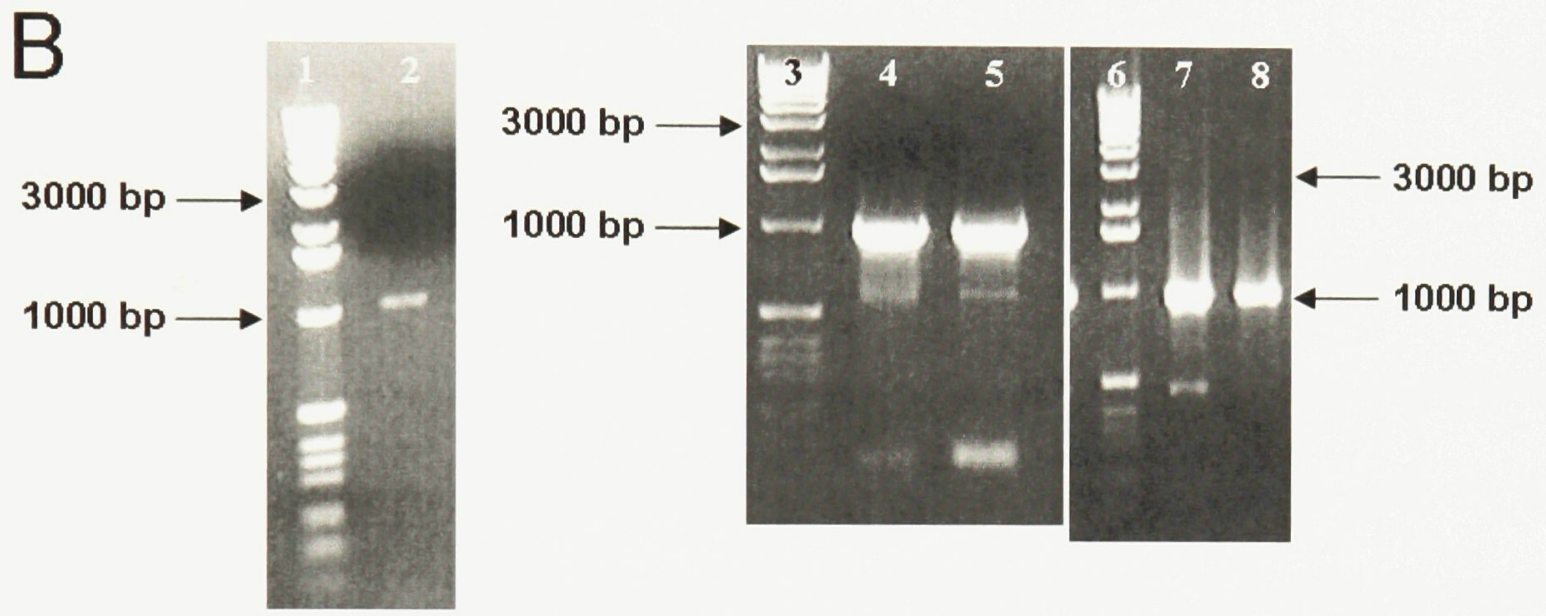

Table 10B. Primers used to obtain LAST cDNA Fragments

\begin{tabular}{|cccc|}
\hline Lane & Fragment & Primer Set & Expected Size \\
\hline 2 & 3' cDNA & $\mathrm{i}+\mathrm{l}$ & $\sim 1000 \mathrm{bp}$ \\
4 & 3' cDNA & $\mathrm{i}+\mathrm{k}$ & $\sim 1000 \mathrm{bp}$ \\
5 & 3' cDNA & $\mathrm{i}+\mathrm{k}$ & $\sim 1000 \mathrm{bp}$ \\
7 & 5' cDNA & $\mathrm{h}+\mathrm{j}$ & $\sim 1000 \mathrm{bp}$ \\
8 & 5' cDNA & $\mathrm{h}+\mathrm{j}$ & $\sim 1000 \mathrm{bp}$ \\
\hline
\end{tabular}

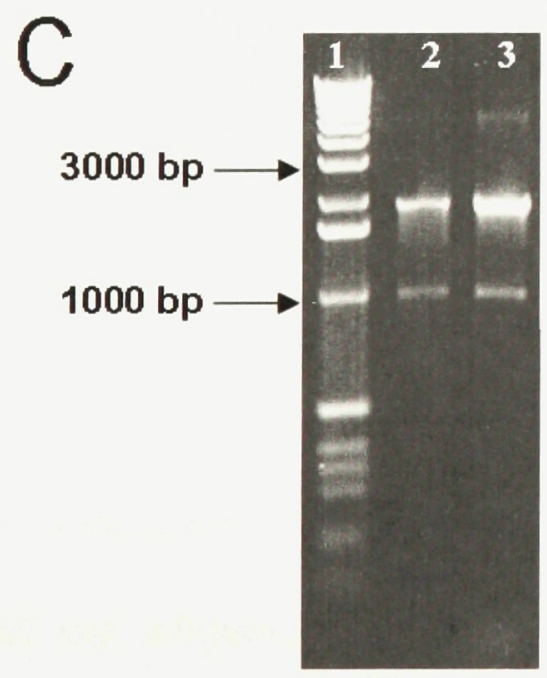

Table 10C. Primers used to obtain full coding sequence of LAST

\begin{tabular}{|cccc|}
\hline Lane & Fragment & Primer Set & Expected Size \\
\hline 2 & Full cDNA & $\mathrm{h}+\mathrm{k}$ & $\sim 1700 \mathrm{bp}$ \\
3 & Full cDNA & $\mathrm{h}+\mathrm{k}$ & $\sim 1700 \mathrm{bp}$ \\
\hline
\end{tabular}




\subsection{Sultr2;1 and Sultr2;2 Expression in A. thaliana}

The expression patterns of the A. thaliana Sultr2;1 and Sultr2;2 genes, which correspond to LAST, were analyzed using the electronic fluorescent pictograph browser (eFP) from the Bioarray Resource tools for A. thaliana (http://bar.toronto.ca). The Sultr2;2 gene is most closely related to the $B$. juncea LAST gene investigated in this study. Absolute expression, using the developmental map dataset, shows the normalized expression values obtained by microarray for the probe corresponding to the Sultr2;2 gene (Fig. 11). The expression data is normalized by removing the top and bottom $2 \%$ of the data in a given experiment (each tissue) and the values are adjusted such that the mean of all the genes in that experiment is equal to 100 . The highest expression was observed in rosette and cauline leaves after bolting. Rosette leaves prior to bolting display low expression, as do the roots prior to bolting. There is no data for roots after bolting. In the influorescence, the flower buds display low expression prior to opening, with expression in the flowers the highest in the sepals. The developing siliques also express Sultr2;2 at a low level.

Similarities between the A. thaliana Sultr2;2 and B. juncea LAST genes include expression in the cauline leaves, and low expression in the roots (Figs. 7, 11). However, the expression profile for the A. thaliana Sultr2;2 gene differs from the expression observed for the B. juncea LAST promoter-GUS reporter constructs in the flowering tissue. The GUS expression, driven by the B. juncea LAST promoter, was present in the developing buds prior to opening, whereas the A. thaliana Sultr2;2 gene appears to express after opening. Also, little expression of the A. thaliana Sultr2;2 gene is observed in the siliques, while strong GUS staining is observed in the siliques of the plants 
expressing pLA:GUS.

The expression of the $A$. thaliana Sultr2;1 gene is considerably stronger than the Sultr2;2 gene (Figs. 11, 12). The expression values for the Sultr2;1 gene reach the highest expression in the hypocotyl, at a value of 709 (Fig. 12). In contrast, the highest value for the Sultr2;2 gene is 239 , in the second leaf of the vegetative rosette (Fig. 11). In relation to its own expression, the Sultr2;2 gene shows relatively high expression in the cauline leaves with a expression value of just 92 (Fig. 11). The tissues with low expression display values of 30 or less. In contrast, the Sultr2;1 gene expresses 1.5-2.5 fold greater than the Sultr2;2 gene in the rosette leaves, pre- and post-bolting (2.4- and 1.7-fold respectively), and in the cauline leaves (1.5 fold) (Fig. 13). However, Sultr2;1 is expressed at lower levels in the flowers than Sultr2;2, particularly in early flower development, where Sultr2;2 is expressed at a level 15-fold greater than the Sultr2;1 gene. Sultr2;2 is also more highly expressed in the shoot apex of the inflorescence than Sultr2;1, while the Sultr2;1 gene is expressed at higher levels than Sultr2;2 in the vegetative shoot apex (Fig. 13). 
Figure 11. The developmental map expression of the A. thaliana LAST gene, Sultr2;2. The figure was created using the Arabidopsis electronic fluorescent pictograph browser from the Bioarray Resource from the University of Toronto (http://bar.utoronto.ca). The signals represent the absolute expression of the Sultr $; 2$ gene as a gradient from red (high expression) to yellow (low expression), which compares each sample to the threshold signal of 140.0. The signal values are the normalized expression data from a given microarray experiment. The expression data is normalized by removing the top and bottom $2 \%$ of the data and the values are adjusted such that the mean is equal to 100 . The highest value recorded in this dataset is 292. Expression values with standard deviations greater than $50 \%$ are masked. 


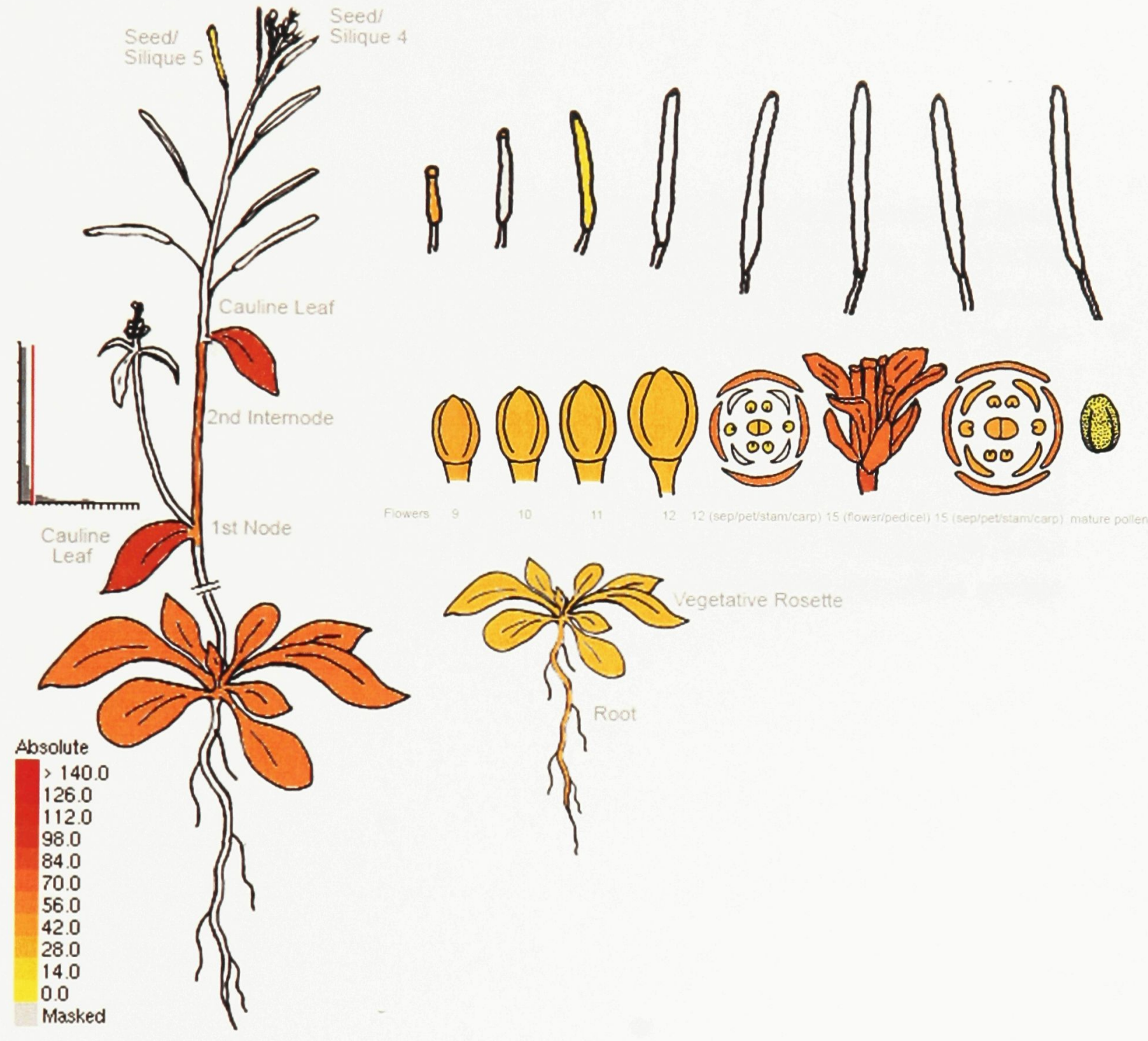


Figure 12. The developmental map expression of the A. thaliana LAST gene, Sultr2;1. The figure was created using the Arabidopsis electronic fluorescent pictograph browser from the Bioarray Resource from the University of Toronto (http://bar.utoronto.ca). The signals represent the absolute expression of the Sultr2; 1 gene as a gradient from red (high expression) to yellow (low expression), which compares each tissue to the threshold signal of 140.0. The signal values are the normalized expression data from a given microarray experiment. The expression data is normalized by removing the top and bottom $2 \%$ of the data and the values are adjusted such that the mean is equal to 100 . The highest value recorded in this dataset is 709 . Expression values with standard deviations greater than $50 \%$ are masked. 


$$
\begin{aligned}
& \text { 㳕 } 11111111 \\
& \text { a }
\end{aligned}
$$


Figure 13. The developmental map comparing expression of the A. thaliana LAST genes, Sultr2;1 and Sultr2;2. The figure was created using the Arabidopsis electronic fluorescent pictograph browser from the Bioarray Resource from the University of Toronto (http://bar.utoronto.ca). The signals represent the comparative expression of the Sultr2;1 gene to the Sultr2;2 gene as a gradient from red (higher expression of Sultr2;1) to blue (lower expression of Sultr2;1). The $\log _{2}$ ratio is the $\log _{2}$ of the Sultr2;1 expression values over the $\log _{2}$ of the Sultr $2 ; 2$ expression values. The $\log _{2}$ ratios are compared to the threshold ratio of 2.0 (4-fold change) to create the colour gradient. 


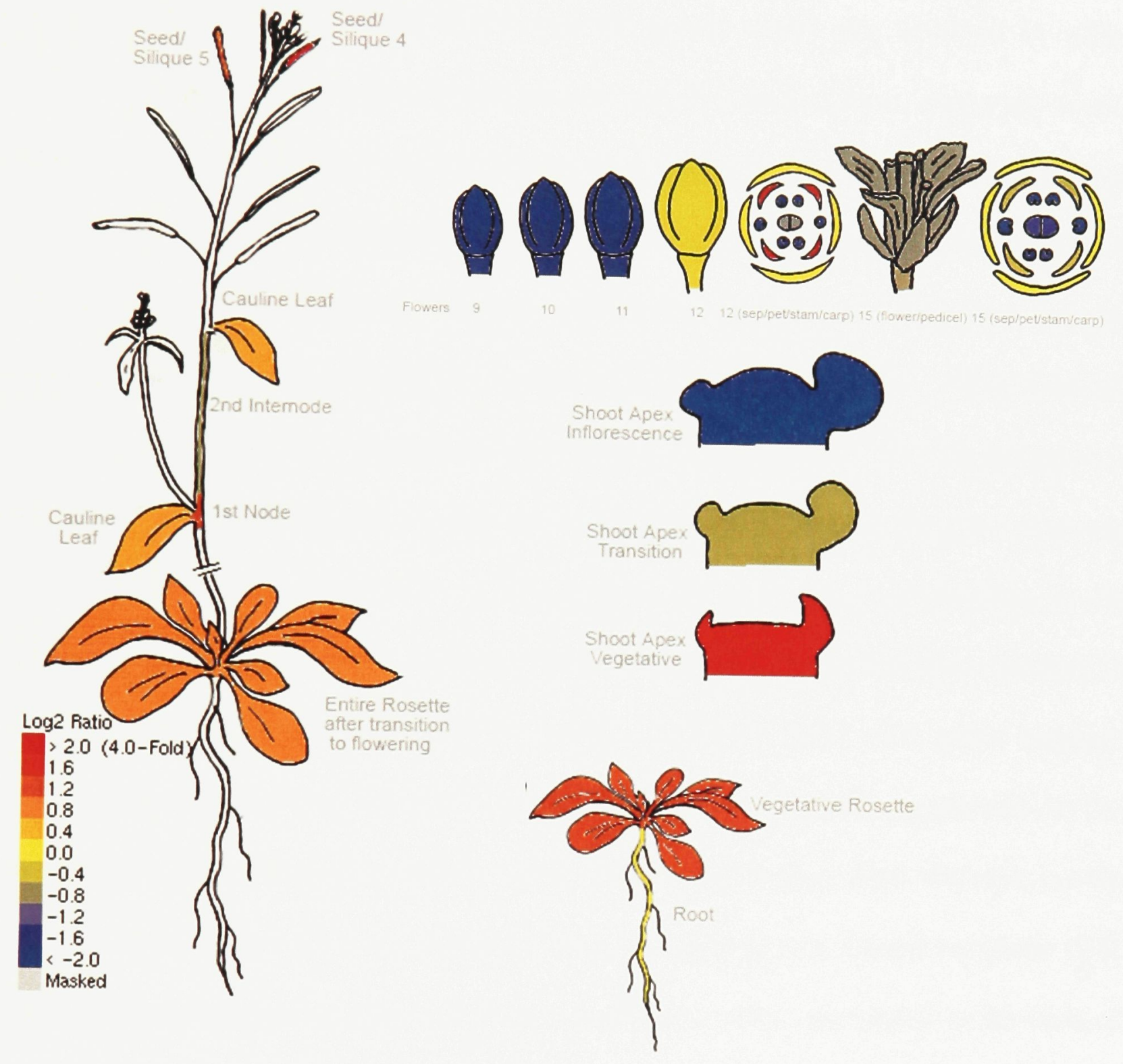




\section{Discussion}

\subsection{Evaluation of Genome Walking}

The objective of this study was the investigation of the transcriptional regulation of the LAST, ATPS, APSR, OASS and PS genes, which are involved in sulfur assimilation and the phytochelatin biosynthetic pathway in B. juncea. Reported studies have routinely demonstrated that $\mathrm{Cd}$ effects the transcriptional regulation of these genes in $B$. juncea, despite the substantial experimental variation, including the concentration of $\mathrm{Cd}$ and the time of treatment, between investigations (Table 1). Therefore, specific interest was placed on probing the regulation of these genes by $\mathrm{Cd}$. Little genomic sequence is available for $B$. juncea and only cDNA sequence data of the targeted genes is present in GenBank. Therefore, genome walking (Genome Walker kit, Clontech) was employed to obtain sequence $5^{\text {' }}$ of the translational start site, corresponding to the promoter region of each gene. Between 3500-4000 bp of sequence has been obtained, in collaboration with Colleen Woodhouse (M.Sc. candidate) for 4 of 8 genes targeted: LAST (this study), GCL, GS and GR (data not shown). Promoter sequences for the remaining 4 genes of interest (ATPS, APSR, OASS and PS) have been obtained, but the goal of 4000 bp has yet to be attained. Genome walking is a ligation-mediated PCR (LM-PCR) technique that relies on adapter sequences, which are ligated to the ends of gDNA fragments. This method is one of several techniques that can be used to obtain DNA sequences bordering a known sequence. Other techniques include randomly primed PCR and inverse PCR, in which a restriction enzyme digested DNA fragment is circularized and reopened at another site (Triglia et al., 1988, Trueba and Johnson, 1996). The element common to each of these techniques is the design of primers specific to a 
known sequence of DNA. Therefore, the likelihood of success in a given genome walking attempt is often determined by the quality of the primers, which must be highly specific to reduce background from non-specific priming and amplification. Such primers are limited by the Genome Walker (Clontech) restrictions of designing nested sets of primers with melting temperatures of $67^{\circ} \mathrm{C}$ within $\sim 150 \mathrm{bp}$ of the $5^{\prime}$ end of the known sequence. Successful PCR can also be complicated by the large genome sizes in some plants, such as the tetraploid genome of $B$. juncea, as genome size has a negative effect on PCR (Garner, 2002). Additionally, the noncoding regions of plant genes, such as the promoter and introns, tend to be difficult regions in which to design highspecificity primers due to the abundance of AT-rich segments in non-coding regions. A. thaliana and Oryza sativa genomes have exon sequences with an average AT content of $57 \%$ and $79 \%$, respectively, while the average AT content of introns are $68 \%$ in A. thaliana and $63 \%$ in O. sativa ( $\mathrm{Yu}$ et al., 2002). The 5' non-coding regions of the sequences obtained in this study all display an AT content of between $60-75 \%$ (Table 10). AT-rich segments also present a challenge for the design of primers with high melting temperatures and GC content, as suggested in the Genome Walking (Clontech) protocol. This difficulty was observed for ATPS where, although lengthy sequence was obtained (two walks of greater than $1000 \mathrm{bp}$ ), it appeared that unspecific sequences were combined during genome walking, which prevented amplification of the entire assembled promoter region. 
Table 10. AT content of sulfur assimilation gene promoter regions

\begin{tabular}{|cc|}
\hline Gene & \% A T $^{a}$ \\
\hline LAST & 60 \\
ATPS & 60 \\
APSR & 60 \\
OASS & 68 \\
GCL & 68 \\
GS & 67 \\
GR & 75 \\
PS1 & 64 \\
PS2 & 72 \\
\hline
\end{tabular}

${ }^{a} \% \mathrm{AT}$ of the non-coding region 5 ' of the translational start site 
The longest promoter sequences obtained were from the LAST, GCL, GS and GR genes, for which only 2, 1, 1 and 2 isoforms, respectively, occur in A. thaliana (Table 1). The remaining genes are present as 2 or more isoforms in A. thaliana (4 ATPS, 3 APSR, 3 OASS and 2 PS). There is a possibility of more isoforms in the $B$. juncea genome than in the A. thaliana genome, or at least more copies of a given isoform, since $B$. juncea is tetraploid. The number of isoforms of a given gene may have a negative effect on genome walking because of unspecific binding by the gene specific primers on the other isoforms, although this depends on the degree of conservation between isoforms (Rampias et al., 2009). Of the group of genes for which the targeted 4000 bp of the promoter region was not attained, the most sequence was obtained for ATPS and PS, with more than $1500 \mathrm{bp}$ each. In the case of ATPS, the initial genome walk employed primers designed 5' of the translational start site, which would be less conserved between isoforms than the coding sequence. This could minimize the negative effect on PCR by other isoforms. On the other hand, PS has 2 isoforms in A. thaliana and thus a successful PCR would be more likely than the APSR and OASS genes with greater than 2 isoforms. The promoter sequences for APSR and OASS were each obtained in genome walks of less than $500 \mathrm{bp}$ of sequence per walk. The more successful genome walks of other genes, with lower isoform number, were upwards of $1500 \mathrm{bp}$ in length suggesting large amplifications were negatively inhibited by isoform number. Collectively, these challenges may explain the lack of sequence obtained for APSR and OASS in genome walking attempts with primers designed from sequence obtained in the first round of genome walking. 
Alternative techniques can provide assistance in obtaining sequences that have proven recalcitrant to standard genome walking. For example, Rampias et al. (2009) have recently reported a unique technique to circumvent the challenge of specificity in LM-PCR. Their approach also relies on the ligation of adapters to gDNA fragments, as in LM-PCR, but limits unspecific binding of gene-specific primers by separating genespecific fragments from the gDNA with a chromatography technique using biotinylated primers. The biotin-labeled, gene-specific primers bound to gDNA fragments are removed from the rest of the gDNA with streptavidin beads. Bound DNA is removed from the beads and PCR can then be performed.

The goal of this study was to obtain $\sim 4000$ bp of sequence $5^{\prime}$ of the translational start site prior to the design of promoter-reporter constructs. This number was chosen as a conservative estimate of the DNA segment expected to comprise a given promoter, as computational prediction of regulatory elements remains one of the most challenging problems in computational biology (Shahmuradov et al., 2003). Eukaryote promoters are divided into the proximal and distal promoters. The proximal promoter sequence generally lies within a couple hundred base pairs, in the 5' direction, of the transcriptional start site and contains most cis-regulatory elements. Meanwhile, the distal promoter sequence may comprise cis-regulatory elements several kilobases away from the transcriptional start site (Shahmuradov et al., 2003). Although most cis-regulatory elements are localized within the proximal promoter, elements within the distal promoter may still act positively or negatively on the expression of a given gene, and therefore it is important not to exclude these elements. For example, Ryu et al. (2009) performed deletion analysis on the promoter of the stress-induced peroxidase gene swpat from 
sweet potato by expressing the GUS reporter gene with seven promoter sequences of between 2374 and 118 bp in length. Expression of each construct was compared to that of the CMV 35S promoter in tobacco protoplasts and expression was observed to be highest (8-fold) with a promoter of 1408 bp in length, while the 374-bp segment still showed 4.5-fold greater expression than the CMV 35S promoter. Interestingly, the GUS expression of the 2374-bp promoter sequence was similar to that of the $374 \mathrm{bp}$ fragment, suggesting the presence of a significant negative regulatory element between 1408 and $2374 \mathrm{bp}$. There have also been several studies probing the promoter regions of specific B. juncea genes. For example, investigation of a 1081-bp segment of a stress-inducible chitinase gene identified a jasmonic-acid-responsive element approximately 700 bp 5 'of the translational start site ( $\mathrm{Wu}$ et al, 2009). A transcriptional response to $\mathrm{Cd}$ and $\mathrm{Ni}$ treatment of a $B$. juncea metal transporter protein (MTP1) gene was identified using promoter:GUS transgenic $B$. juncea plants with 983 bp of the promoter region. The relative expression matched data from northern blots of the MTP1 mRNA, and was unchanged by the presence of additional sequence (1561 and $1786 \mathrm{bp}$ ) 5' of the translational start site (Muthukumar et al., 2007). In contrast, deletion analysis of a $B$. juncea glutathione S-transferase promoter showed that both 310 bp and 756 bp immediately $5^{\prime}$ of the translational start site was sufficient for GUS expression in the roots and shoots of $A$. thaliana under control conditions. However, relative expression, as determined by a fluorometric GUS assay, was lowered $\sim 2.5$-fold when 1145 bp and $1520 \mathrm{bp}$ of the promoter region was used, while expression of GUS under a 2623-bp promoter segment resulted in the same relative expression as the 310 and 756 bp constructs. A significant increase expression (under 2-fold) was observed in plants 
expressing GUS with the 2623 bp promoter region of GST when treated with $\mathrm{H}_{2} \mathrm{O}_{2}$, salicylic acid and 1-aminocyclopropane-1-carboxylate (Gong et al. 2005). Together, these studies illustrate that cis-regulatory elements are often found within $1000 \mathrm{bp}$, but that elements further away may change relative levels of that expression. Regulatory elements are also found in introns and in sequences 3' of the translational stop site (Rose, 2004; Xie et al., 2005). In the current study, approximately 3500 bp of promoter sequence from the $B$. juncea LAST gene was investigated, via promoter-reporter constructs in $A$. thaliana, while $\sim 1500$ bp was used for the corresponding PS1 and PS2 constructs. Although 1500 bp may not contain all regulatory elements, the PS promoters likely contain the majority of any cis-regulatory elements to display appropriate patterns of expression. Further analysis of expression patterns for a given gene in $B$. juncea, such as Northern blots, are necessary to determine if expression patterns of GUS in $A$. thaliana, driven by a $B$. juncea promoter, are similar in the two species.

\subsection{Identification of Additional PS Isoform}

Since B. juncea is a tetraploid species and multiple isoforms exist in A. thaliana for the genes targeted in this study, there was an increased likelihood of genome walking in separate isoforms and this was observed in the case of PS. Two distinct 5' regions were identified, and confirmed via diagnostic PCR and sequence analysis, for the PS gene and were designated PS1 and PS2 (Fig. 5). The sequences 3' of the translational start site, excluding introns, obtained for each PS isoform share high sequence identity with the PS1 B. juncea cDNA sequence available in Genbank (AJ278627) (100\% and 93\% for PS1 and PS2, respectively) (Heiss et al., 2003). However, the PS1 and PS2 
genomic sequences share just $84 \%$ sequence identity, due to the presence of introns, which are less conserved. Although the PS gene reported by Heiss et al. (2003) is a cDNA sequence and contains no introns, it does include 106 bp of 5'-UTR sequence, which is $89 \%$ identical to the PS1 gene identified in this study. In contrast, no sequence identity was observed with PS2. Therefore, given the higher sequence identity within the coding sequence and the similarity in the 5' UTR, the PS1 gene sequence in this study corresponds to the $B$. juncea PS1 gene in Genbank, while the PS2 gene represents an unreported PS isoform of $B$. juncea. The discovery of a second isoform is not surprising as A. thaliana has two PS isoforms, and L. japonicus contains three (Ramos et al., 2007).

\subsection{Construction of Promoter:GUS Lines in A. thaliana}

The use of GUS as a reporter gene is widespread in plant molecular biology, and was chosen in this study for analysis of the promoters obtained by genome walking (An et al., 2005, Huttly, 2009). Other commonly used reporter genes, suitable for plants, include luciferase and GFP. The GUS reporter system possesses numerous advantages, such as ease of handling and strong stability and activity under varying $\mathrm{pH}$ and ionic conditions. Furthermore, visualization of GUS staining in plant tissues requires only a light microscope, in contrast with the GFP and LUC systems, which require additional equipment such as fluorescence filters and high-sensitivity charge-coupled devices, respectively. Although an advantage of the GFP and LUC systems is that live tissues can be visualized without the histochemical staining required for GUS (Huttly, 2009), a direct comparison of GFP and GUS, has demonstrated that GUS has a longer half life in tissue after induction than GFP (Kavita and Burma, 2008). Therefore, GUS is an appropriate 
reporter gene for systems in which dynamic changes in temporal expression are not being investigated. Also, weak promoters are effectively analyzed with GUS, since the longer half life allows for buildup of the GUS protein, allowing visualization.

A series of seven promoter-reporter constructs were designed for the analysis of the B. juncea LAST, PS1 and PS2 promoter sequences. Four of these constructs were truncations of the LAST promoter, while the other three constructs comprise the fulllength sequences for LAST (3771 bp), PS1 (1485 bp) and PS2 (1491 bp) (Table 8). Each promoter sequence was inserted at the BamHI and NcoI sites of pGCO:GUS, thereby replacing the CO promoter in this vector. The resulting promoter:GUS plasmids contain the promoter:GUS sequence, along with the Bar gene, which confers resistance to PPT and the herbicide Basta, driven by the Nos promoter, between the left and right T-DNA border sequences. The Agrobacterium-mediated transformation of A. thaliana inserts the sequence of DNA present between the left and right border sequences of the plasmids randomly into the A. thaliana genome. This can lead to positional effects, due to random insertion, such as silencing and expression of the GUS gene by cryptic cis-regulatory elements (Huttly, 2009). Alterations in chromatin structure can silence plant genes. Silenced plant genes are often located in regions of cytosine-methylation. Should a transgene be inserted in an actively methylated region of chromatin, expression of the transgene could be silenced (Kloti et al., 2002). Also, expression can be caused by the promoter for the selectable marker, in this case the NOS promoter for the expression of the Bar gene (Huttly, 2009). Therefore, to circumvent the potential of aberrant expression, a minimum of 10 independent homozygous $\mathrm{T} 3$ lines for each construct were tested (Table 9). 
Previous studies have demonstrated mixed success for the investigation of nonnative promoter activity in A. thaliana. For example, Wu et al. (2009) expressed GUS in A. thaliana under the control of a chitinase promoter from the closely related B. juncea, also a member of the Brassicaceae family, and found unaltered expression between the two species, as reverse transcriptase-PCR confirmed that expression was similar in $B$. juncea. Promoters from more distantly related species have also been shown to demonstrate native expression patterns in A. thaliana. For example, the soybean lectin3 promoter drives the expression of GUS in A. thaliana the same as it does in soybean (Saeed et al., 2008). However, Howarth et al. (2009) found that the sulfur deficiencyinduced gene (sdi1) promoter sequence from wheat fails to express GFP in A. thaliana. These studies demonstrate that there is conservation in function for cis-regulatory elements, particularly between closely related species, but that this conservation is not exclusive.

\section{4. pLA:GUS Expression in A. thaliana}

Expression of GUS, driven by the $B$. juncea LAST promoter in A. thaliana, was localized to new leaves developing at the shoot apex and to developing buds after bolting, with no expression in the roots (Fig. 7). GUS staining was also observed at the base of developing flowers, and in developing siliques of 4-5 week-old plants and was strongest in siliques that had yet to form distinct seeds. The truncation constructs revealed that this pattern of expression required up to $\sim 1500 \mathrm{bp}$ of the region $5^{\prime}$ of the translational start site, as the pLA-Tru1:GUS plants showed that a 400 -bp segment of the promoter region is insufficient to produce the expression pattern observed for the full-length $(3771 \mathrm{bp})$, 
LA-Tru3 (1535 bp) and LA-Tru4 (2198 bp) constructs. However, expression was observed in several LA-Tru1 lines, suggesting that the $\sim 400$-bp sequence of this construct may comprise the minimal promoter elements, but requires additional regulatory sequences to initiate transcription. Based on the common expression patterns of the fulllength, LA-Tru3 and LA-Tru4 lines, these regulatory sequences must be present in the $\sim 1100$ bp region between the Tru1 (436 bp) and Tru3 (1535 bp) sequences.

\subsubsection{Comparisons of LAST Expression in A. thaliana and B. juncea}

The $B$. juncea LAST coding sequence shares $88 \%$ and $67 \%$ sequence identity to the A. thaliana Sultr2;2 and Sultr2;1 coding sequences, respectively. Northern blot analysis has shown that the LAST gene is expressed in both the roots and leaves of $B$. juncea plants (Heiss et al., 1999). In A. thaliana, the Sultr2;2 gene is expressed predominately in the shoots, but expression is also observed in the roots at lower levels (Takahashi et al., 2000). In contrast, expression of the B. oleracea Sultr2;2 gene is localized to the roots, with little expression observed in the leaves, while the B. oleracea Sultr2; 1 gene is expressed primarily in the leaves (Buchner et al., 2004a). These results suggest that the expression patterns of LAST isoforms vary between species in the Brassicaceae family. In this study, the pattern of GUS expression in A. thaliana, driven by the $B$. juncea LAST promoter, was similar to that of the A. thaliana Sultr2;2 gene (Fig. 7), as expression was observed in the shoots, but not in the roots. The eFP browser application used to analyze A. thaliana microarray data showed relatively higher expression of the Sultr2;2 gene in the flowering parts (Fig. 11). Similarly, GUS expression in the full-length, LA-Tru3 and LA-Tru4 lines was localized to the flowering 
regions. The similarities in GUS expression driven by the B. juncea LAST promoter region to Sultr $2 ; 2$ in A. thaliana, instead of the expression patterns of LAST observed in B. juncea, suggests differences in trans-acting factors for LAST expression between $B$. juncea and A. thaliana. Transcription factors required for cell and tissue specific expression of LAST in the roots may not be the same in A. thaliana and B. juncea roots. Activators could be down regulated, or a repressor may be expressed in A. thaliana roots. Alternatively, an activator could be present in $B$. juncea roots.

\subsubsection{In silico and Experimental Evidence of cis-Regulatory Elements}

Currently, the most common computational analysis of plant promoter sequences is the identification of cis-regulatory elements using databases such as PLACE (Higo et al., 1999) and PlantCARE (Lescot et al., 2002), which search a query sequence for previously reported cis-regulatory sequences. These databases provide large datasets with short sequences of 4-6 nucleotides, but little information on other sequence requirements, such as those for proper position relative to the transcriptional start site, or the presence of additional elements. Alternatively, some programs, such as Promomer from the Bio-Array Resource (http://bar.utoronto.ca), attempt to identify over-represented n-mer "words" in a given promoter or co-regulated promoters compared to a background set of promoter sequences. These over-represented sequences can then be compared to sequences of elements present in PLACE and PlantCare databases to identify potential regulatory elements. However, the challenge remains that these databases provide sufficiently large lists of potential target sequences to make the experimental testing of each putative element far too time consuming. Therefore, it is necessary to have some 
previous genetic evidence for the regulation of a promoter. In this study, the LAST promoter was analyzed using the PLACE database (Higo et al., 1999) (Fig. 6). Previous studies have shown a decrease in mRNA transcript levels in the roots, but not the shoots, of the B. juncea LAST gene after treatment with $25 \mu \mathrm{M} \mathrm{Cd}$ for $48 \mathrm{~h}$ (Heiss et al., 1999). Therefore, metal responsive elements were targeted for the truncation constructs of the LAST promoter. The promoter region of the $B$. juncea LAST gene was found to contain both CuRE and SuRE in similar numbers to its $A$. thaliana homolog, Sultr2;2. The Sultr2;1 gene in A. thaliana, however, contained 18 less CuRE sites than the B. juncea LAST promoter. CuRE elements are required for $\mathrm{Ni}^{2+}$ induction of the genes cytochrome $\mathrm{c}_{6}$ (Cyc6) and coprogen oxidase $1(\mathrm{Cpx} 1)$ in Chlamydomonas reinhardtii, in addition to their ability to induce Cyc6 and Cpx 1 during Cu deficiency (Quinn et al., 2003). Cobalt ions have been shown to similarly induce the Cyc 6 and $\mathrm{Cpx} 1$ genes, but the involvement of the CuRE sites in induction by Co was not tested (Quinn et al., 2003). Therefore, the LAST gene may be regulated by some of the CuRE sites by $\mathrm{Ni}^{2+}$ or copper deficiency and further analysis could reveal regulation with other metal ions, such as $\mathrm{Cd}$. It should be noted, however, that the CuRE sequence identified is a core sequence of $4 \mathrm{bp}$ in length and would occur 14 times in the 3771 bp promoter region given random chance. Also, although this core sequence is required for copper deficiency and $\mathrm{Ni}^{2+}$ response, other sequence or spatial requirements may be necessary for the full response. Therefore, it is possible, even likely, that the full set of $14 \mathrm{CuRE}$ sequences identified in the 3771-bp region 5 ' of the translational start site of the $B$. juncea LAST gene are not required for a given response. The truncation constructs can provide a tool for the analysis of functional CuRE sites in the LAST promoter region. Zero, 2, 3, 8 and 14 CuRE elements 
are within the promoter regions of the LA-Tru1 (436 bp), LA-Tru2 (890 bp), LA-Tru3 (1535 bp), LA-Tru4 (2198 bp) and full-length LAST (3771 bp) sequences. Interestingly, 8 CuRE sites are present within $\sim 1000$ bp between 2010 and 2925 bp 5' of the translational start site. Further testing of the LAST promoter constructs on other metals, such as $\mathrm{Ni}^{2+}$, may reveal a response requiring one or more of the CuRE sites. In addition to the large number of CuRE sites, two putative MRE sites (MRE-like: GCCTGCA and novel MRE: ATTCCAAAATTC) were identified in the $B$. juncea LAST promoter, while neither were found in the two $A$. thaliana LAST promoters. The LAST truncation constructs were based around these two putative MRE sites (Table 8). These elements are longer in sequence, and thus there is a less statistical likelihood of occurring in the sequence (every $1684 \mathrm{bp}$ for the 7-bp MRE-like and $1.68 \times 10^{7} \mathrm{bp}$ for the 11-bp novelMRE). Each of these MRE sites were identified in the stress-related SR2 gene from Phaseolus vulgaris and were found to be required for metal induced transcription of SR2 after treatments with several metal ions, including $\mathrm{Cd}^{2+}$ (Qi et al., 2007). Unfortunately, no data is yet available on the pLA-Tru2:GUS lines, as they are in the T2 stage and the homozygous lines are not yet available. The LA-Tru2 sequence contains the MRE-Like sequence only, and would thus aid in determining specific roles for each of the MRE sequences, as the pLA:GUS, pLA-Tru4:GUS and pLA-Tru3:GUS constructs all contain both putative MRE sites (Table 8). The Cd treatment of the pLA:GUS, pLA-Tru4:GUS and pLA-Tru3:GUS showed no GUS expression at the higher concentrations of Cd (50 and $100 \mu \mathrm{M} \mathrm{CdCl}_{2}$ ) tested (Fig. 9). Plants were severely stressed at these concentrations and lack of expression could be due to the stress of the $\mathrm{Cd}$ treatment rather than a change in gene regulation (Fig. 8). However, following treatment with $10 \mu \mathrm{M} \mathrm{CdCl}_{2}$ for 3 weeks 
the pLA-Tru4:GUS and pLA-Tru3:GUS lines showed staining in the leaves while the pLA:GUS plants contained no staining (Fig. 9). The staining observed was more extensive than under control conditions, with staining in more developed leaves (Figs. 7, 9). These results suggest that the LAST promoter may contain metal regulatory elements, but that elements capable of reducing expression exist between 2198 and 3771 bp downstream of the translational start site. The observed down-regulation could explain the decrease in transcripts observed by Heiss et al. (1999) in the roots of $\mathrm{Cd}$ treated B. juncea plants. However, it is interesting that negative regulation was observed in the shoots in the current study, where Heiss et al. (1999) observed no changes in LAST transcript levels. The treatment conditions were different between the two studies, as Heiss et al. (1999) treated 5-week-old B. juncea plants with $25 \mu \mathrm{M} \mathrm{Cd}$ for $48 \mathrm{~h}$ in hydroponics, which did not cause noticeable stress, while in this study A. thaliana plants were grown continuously for 3 weeks on $10 \mu \mathrm{M} \mathrm{Cd}$ plates and were chlorotic and stunted (Fig. 8). Further analysis of LAST gene expression in B. juncea may reveal negative regulation within the shoots when treated for longer duration. Heiss et al. (1999) suggested that decreased expression of LAST in the roots would retain sulfate in the roots rather than transporting sulfate through the vasculature to the shoots and provide a means for the plant to tolerate $\mathrm{Cd}$ at its source. A similar process may occur in the shoots, whereby LAST expression is decreased to allow sulfate assimilation within vasculature cells directly affected by $\mathrm{Cd}$.

The LAST promoter also contained 5 SuRE core sites, including 4 within 2000 bp from the translational start site. The SuRE core is a 5 bp sequence and would be expected to be present 3.7 times in the full LAST promoter ( $3771 \mathrm{bp}$ ), given random 
chance. Of these 5 SuRE core sequences, 2 contained the functional 7-bp functional motif. SuRE sites were discovered in the A. thaliana HAST gene Sultr1; 1 , by deletion analysis of the promoter using luciferase as a reporter gene, and were determined to be required for the increase HAST in expression when plants were grown without sulfur (Maruyama-Nakashita et al., 2005). The 7-bp sequence contains a 6-bp known auxin response factor (ARF) binding site. Base substitution of the 7-bp motif confirmed the importance of the 5-bp core sequence as well as the function. Further analysis of sulfurresponsive promoters in A. thaliana showed that all 15 genes identified as sulfur responsive by microarray, contained the SuRE core sequence, and 13 contained the full ARF binding site (Maruyama-Nakashita et al., 2005). The SuRE sequences have also been found in a sulfate deficiency-induced gene, sdil, in wheat, which suggests these sequences are conserved in the response to sulfate deficiency (Howarth et al., 2009). Both of the LAST genes, Sultr2;1 and Sultr2;2, in A. thaliana increase their expression under conditions of sulfate deprivation, as determined by reverse transcriptase-PCR. However, the increase in expression is observed in distinct tissues. Expression of the Sultr2;1 gene is increased in the roots, while expression of Sultr2;2 is increased in the leaves (Takahashi et al., 2000). In this study, GUS expression of the $B$. juncea LAST gene showed no changes when plants were grown without sulfate salts in the media. However, the GUS staining technique employed only allows changes in the spatial expression to be adequately observed, while relative changes in expression in a given tissue would be difficult to observe. Therefore, expression may have increased in the leaf tissue where expression was also observed under control conditions. If the $B$. juncea 
LAST gene is regulated in a manner similar to the Sultr $2 ; 2$ gene in A. thaliana, this pattern would be expected.

\subsection{LA:pBAR1: Future Directions}

The LA:pBAR1 construct was created to study the effects of the ectopic expression of the $B$. juncea LAST gene, under its native promoter, in A. thaliana. The resulting plants could be studied for changes in sulfur metabolism and response to treatment with toxic metals. The LA:pBAR1 construct was successfully created by inserting the $B$. juncea LAST coding sequence and promoter into the pBAR1 vector (Fig. 10). The coding sequence was obtained in two fragments and reassembled by overlap PCR. Since the B. juncea LAST gene is only available as a partial mRNA in Genbank, confirmation of sequence identity was determined by alignment with the Genbank sequence and the sequence obtained during genome walking. The newly acquired coding sequence confirms that the B. juncea LAST gene is most closely related to the Sultr2;2 genes in A. thaliana and B. oleracea.

Targeting sulfate transport represents a unique approach to engineering plants for phytoremediation applications, as most reports to date have focused on genes toward the end of the phytochelatin biosynthetic pathway. Sulfate transport is the first step in sulfur metabolism and altering the expression of sulfate transporters could change the sulfur status of the entire plant or of specific target tissues. Several important chelator compounds are derived from sulfur-containing amino acids. Metallothioneins are small cysteine-rich proteins, while PC and nicotianamine, a Fe chelator derived from $S$ adenosyl methionine, are enzymatically synthesized by PS and nicotianmine synthase, 
respectfully (Freisinger, 2008; Roje, 2006). A current problem encountered in developing an effective phytoremediator is the negative effects associated with the increased expression of enzymes in the PC biosynthetic pathway. The majority of studies have relied upon strong, constitutive promoters to drive the expression of the genes of interest. This has led to adverse effects, such as the observed Cd-hypersensitivity of PSexpressing A. thaliana plants (Gasic and Korban, 2007). Studies of transgenic B. juncea plants constitutively expressing GCL and GS have shown only small improvements in Cd tolerance, and these plants were stressed under control conditions (Zhu et al., 1999a, Zhu et al., 1999b). These effects are likely due to adversely changing the flux through the sulfur assimilation pathway, and related pathways, in the cell. Wawrzynski et al. (2006) attempted to alleviate this problem by creating transgenic tobacco lines overexpressing three genes, all under the control of the CaMV 35S promoter: serine acetyltransferase (SAT, part of the cysteine synthase complex), GS and PS. The resulting plants did not demonstrate improved tolerance, as plant fresh weight remained the same as wild-type, but the transgenic plants did accumulate more $\mathrm{Cd}$ in the roots than wild-type after 3 weeks of $15 \mu \mathrm{M}$ Cd treatment. Another study attempted tissue-specific expression of the AtPS1 gene under control of the leaf-specific chlorophyll a/b binding protein promoter in wild-type $A$. thaliana plants (Peterson and Oliver, 2006). Root length was significantly higher in transgenic plants than wild-type when germinated and grown in 25 or $50 \mu \mathrm{M}$ $\mathrm{Cd}^{2+}$ for 10 days; however $\mathrm{Cd}$ accumulation in the shoots or roots was not increased as a result of these treatments. These studies suggest more research into the regulation of this pathway is needed to develop a refined approach to increasing Cd-tolerance in plants. 


\section{CONCLUSIONS}

Phytoremediation represents a unique, cost-effective approach to cleaning up metal-contaminated soils. It would ideally rely upon fast growing plants, such as $B$. juncea, that can tolerate and accumulate, in the above-ground biomass, toxic metals, such as cadmium. Many studies to date have focused on expressing enzymes at the end of the phytochelatin biosynthetic pathway under strong, constitutive promoters, such as CaMV 35S. The resulting plants, although displaying increased metal tolerance, are often stressed, compared to wild type plants, under control conditions and demonstrate small, ineffective changes in the accumulation of metals in the shoots. Understanding of the genetic controls of sulfur assimilation in the hyperaccumulater $B$. juncea will allow more refined approaches for engineering effective phytoremediators. Promoter regions of LAST, ATPS, APSR, OASS and PS were obtained by genome walking to probe the transcriptional control of these regions in A. thaliana. In the process, an unreported isoform of PS was identified. Promoter:GUS plasmids were constructed for the two PS isoforms (PS1 and PS2) and 5 LAST constructs. Four of the LAST constructs comprise truncated regions of the LAST promoter region, strategically divided to test the function of two putative MRE sites found by bioinformatic analysis. The untreated A. thaliana lines expressing LAST promoter:GUS constructs demonstrated GUS expression in the new leaves of 3 week-old plants, and in the cauline leaves, flowers and siliques of 4 week-old plants. The regulatory elements responsible for this pattern of expression were localized to a region between 436 and 1535 bp of the promoter. Sulfate deprivation and treatments of 1,50 and $100 \mu \mathrm{M}$ Cd showed no changes in GUS expression driven by the LAST promoter regions. However, increased staining compared to the control in the 
leaves of 3 week-old plants treated with $10 \mu \mathrm{M} \mathrm{Cd}$ was observed in plants expressing GUS with 2198 and 1535 bp segments of the LAST promoter region. This pattern was not observed with 3771 bp segment of the promoter region, suggesting a negative regulatory element exists between 2198 and $3771 \mathrm{bp}$. 


\section{REFERENCES}

Ahmad A., Khan I., Anjum N., Abrol Y., and Iqbal M. (2005). Role of sulphate transporter systems in sulphur efficiency of mustard genotypes. Plant Sci. 169, 842-846.

An G., Jeong D.H., J K.H. and Lee S. (2005). Reverse genetic approaches for functional genomics of rice. Plant Mol. Biol. 59, 111-123.

Banuelos G., Terry N., Leduc D.L., Pilon-Smits E.A.H., and Mackey B. (2005). Field trial of transgenic Indian Mustard plants shows enhanced phytoremediation of seleniumcontaminated sediment. Environ. Sci. Technol. 39, 1771-1777.

Bennett L.E., Burkhead J.L., Hale K.K., Terry N., Pilon M. and Pilon-Smits E.A.H. (2003). Analysis of Transgenic Indian Mustard Plants for Phytoremediation of MetalContaminated Mine Tailings. J. Environ. Qual. 32, 432-440.

Bick J.A., Aslund F., Chen Y. and Leustek. (1998). Glutaredoxin function for the carboxyl-ternminal domain of the plant-type 5'-adenylylsulfate reductase. Proc. Natl. Acad. Sci. USA. 95, 8404-8409.

Bork C., Schwenn J.D., and Hell R. (1998). Isolation and characterization of a gene for assimilatory sulfite reductase from Arabidopsis thaliana. Gene 212, 147-153.

Boyd R.S. and Martens S.N. (1994). Nickel hyperaccumulated by Thlaspi montanum var. montanum is acutely toxic to an insectherbivore. OIKOS. 70, 21-25.

Boyd R.S, Shaw J.J. and Martens S.N. (1994). Nickel hyperaccumulation defends Streptanthus polygaloides (Brassicaceae) against pathogens. Am. J. Bot. 81, 294-300.

Buchner P., Stuiver C.E.E., Westerman S., Wirtz M., Hell R., Hawkesford M.J., and De Kok L.J. (2004a). Regulation of Sulfate Uptake and Expression of Sulfate Transporter Genes in Brassica oleracea as Affected by Atmospheric $\mathrm{H}_{2} \mathrm{~S}$ and Pedospheric Sulfate Nutrition. Plant Physiol. 136, 3396-3408.

Buchner P., Takahashi H., and Hawkesford M.J. (2004b). Plant sulphate transporters: co-ordination of uptake, intracellular and long-distance transport. J. Exp. Bot. 55, 17651773 .

Chaney R.L. (1983). Plant uptake of inorganic waste. In Land Treatment of Hazardous Wastes, ed. JE Parr, PB Marsh, JM Kla, pp. 50-76. Park Ridge, IL: Noyes Data Corp.

Clemens, S., Kim, E.J., Neumann, D. and Schroeder, J.I. (1999) Tolerance to toxic metals by a gene family of phytochelatin synthases from plants and yeast. EMBO J. 18, 3325 .

Cobbett C.S. and Goldsborough P.B. (2002) Phytochelatins and metallothioneins: roles in heavy metal detoxification and homeostasis. Annu Rev Plant Biol. 53, 159-182. 
Cobbett C.S., May M.J., Howden R. and Rolls B. (1998). The glutathione-deficient, cadmium-sensitive mutant, cad2-1, of Arabidopsis thaliana is deficient in gammaglutamylcysteine synthetase. Plant J. 16, 73-78.

Cumming M., Leung S., McCallum J., and McManus M.T. (2007). Complex formation between recombinant ATP sulfurylase and APS reductase of Allium cepa (L.). FEBS Lett. 581, 4139-4147.

Dominguez-Solis J.R., Gutierrez-Alcala G., Vega J.M., Romero L.C., and Gotor C. (2001). The cytosolic O-acetylserine(thiol)lyase gene is regulated by heavy metals and can function in cadmium tolerance. J. Biol. Chem. 276, 9297-9302.

Foyer C.H., Souiau N., Perret S., Lelandais M., Kunert K.J., Pruvost C., and Jouanin L. (1995). Overexpression of glutathione reductase but not glutathione synthetase leads to increases in antioxidant capacity and resistance to photoinhibition in poplar trees. Plant Physiol. 109, 1047-1057.

Freeman J.L., Persans M.W., Nieman K., Albrecht C., Peer W., Pickering I.J. and Salt D.E. (2004). Increased glutathione biosynthesis plays a role in nickel tolerance in thlaspi nickel hyperaccumulators. Plant Cell 16, 2176-2191.

Freisinger E., (2008). Plant MTs-long neglected members of the metallothionein superfamily. Dalton Trans. 47, 6663-6675.

Fusco N., Micheletto L., Dal Corso G., Borgato L., and Furini A. (2005). Identification of cadmium-regulated genes by cDNA-AFLP in the heavy metal accumulator Brassica juncea L. J. Exp. Bot. 56, 3017-3027.

Garner T.W.J. (2002). Genome size and microsatellites: the effect of nuclear size on amplification potential. Genome. 45, 212-215.

Gasic K. and Korban S.S. (2007). Expression of Arabidopsis phytochelatin synthase in Indian mustard (Brassica juncea) plants enhances tolerance for $\mathrm{Cd}$ and $\mathrm{Zn}$. Planta. 225, $1277-1285$.

Gong H., Hu W.W., Jiao Y., and Pua E.C. (2005) Molecular characterization of a Phiclass mustart (Brassica juncea) gluathione s-transferase gene in Arabidopsis thaliana by 5'-deletion analysis of its promoter. Plant Cell Rep. 24, 439-447.

Grotz N., Fox T., Connolly E., Park. W., Guerinot M.L., and Eide D. (1998) Identification of a family of zinc transporter genes from Arabidopsis that respond to zinc deficiency. Proc. Natl. Acad. Sci. USA. 95, 7220-7224.

Grill E., Winnacker E.L., and Zenk M.H. (1987). Phytochelatins, a class of heavymetal-binding peptides from plants, are functionally analogous to metallothioneins. Proc. Natl. Acad. Sci. USA. 84, 439-443. 
Hall J.L. (2002). Cellular mechanisms for heavy metal detoxification and tolerance. J. Exp. Bot. 53, 1-11.

Hall J.L. and Williams L.E. (2003). Transition metal transporters in plants. J. Exp. Bot. 54, 2601-2613.

Harada E., Yamaguchi Y., Koizumi N., and Hiroshi S. (2002). Cadmium stress induces production of thiol compounds and transcripts for enzymes involved in sulfur assimilation pathways in Arabidopsis. J. Plant Physiol. 159, 445-448.

Hatzfeld Y., Lee S., Lee M., Leustek T., and Saito K. (2000). Functional characterization of a gene encoding a fourth ATP sulfurylase isoform from Arabidopsis thaliana. Gene 248, 51-58.

Heiss S., Schafer H.J., Haag-Kerwer A., and Rausch T. (1999). Cloning sulfur assimilation genes of Brassica juncea L.: cadmium differentially affects the expression of a putative low-affinity sulfate transporter and isoforms of ATP sulfurylase and APS reductase. Plant Mol. Biol. 39, 847-857.

Heiss S., Wachter A., Bogs J., Cobbett C., and Rausch T. (2003). Phytochelatin synthase (PCS) protein is induced in Brassica juncea leaves after prolonged Cd exposure. J. Exp. Bot. 54, 1833-1839.

Hellens R.P., Edwards E.A., Leyland N.R., Bean S., and Mullineaux P.M. (2000). pGreen: a versatile and flexible binary $\mathrm{Ti}$ vector for Agrobacterium-mediated plant transformation. Plant Mol. Biol. 42, 819-832.

Herbette S., Taconnat L., Hugouvieux V., Piette L., Magniette M.L., Cuine S., Auroy P., Richaud P., Forestier C., Bourguignon J., Renou J.P., Vavasseur A., and Leonhardt N. (2006). Genome-wide transcriptome profiling of the early cadmium response of Arabidopsis roots and shoots. Biochemie. 88, 1751-1765.

Hesse H., Lipke J., Altmann T. and Hofgen R. (1999). Molecular cloning and expression analyses of mitochondrial and plastidic isoforms of cysteine synthase (Oacetylserine(thiol)lyase) from Arabidopsis thaliana. Amino Acids 16, 113-131.

Hicks L.M., Cahoon R.E., Bonner E.R., Rivard R.S., Sheffield J., and Jez J.M. (2007). Thiol-based regulation of redox-active glutamate-cysteine ligase from Arabidopsis thaliana. Plant Cell 19, 2653-2661.

Higo K., Ugawa Y., Iwamoto M. and Korenaga T. (1999). Plant cis-acting regulatory DNA elements (PLACE) database: 1999. Nucleic Acid Res. 27, 297-300.

Higuchi, R. (1990). Recombinant PCR. In Innis, M.A., Gelfand, D.H., Sninsky, J.J., White, T.J., eds. PCR protocols: a guide to methods and applications. pp. 177-183. Academic Press. San Diego. 
Ho S.V., Sheridan P.W., Athmer C.J., Heitkamp M.A., Brackin J.M., Weber D. and Brodsky P.H. (1995). Integrated In Situ Soil Remediation Technology: The Lasagna Process. Environ. Sci. Technol. 29, 2528-2534.

Howarth J.R., Parmar S., Barraclough P.B., and Hawkesford M.J. (2009). A sulphur deficiency-induces gene, sdil, involved in the utilization of stored sulphate pools under sulphur-limiting conditions has potential as a diagnostic indicator of sulphur nutritional status. Plant Biotech. J. 7, 200-209.

Howden R., Andersen C.R., Goldsbrough P.B. and Cobbett C.S. (1995). A CadmiumSensitive, Glutathione-Deficient Mutant of Arabidopsis thaliana. Plant Physiol. 107, 1067-1073.

Howden R., Goldsbrough P.B., Andersen C.R. and Cobbett C.S. (1995b). Cadmiumsensitive, cadl mutants of Arabidopsis thaliana are phytochelatin deficient. Plant Physiol. 107, 1059-1066.

Huang, X. and Madan, A. (1999). CAP3: A DNA sequence assembly program. Genome Res. 9, 868-877.

Huttly, A. (2009). Reporter genes. Methods Mol Biol. 478, 39-69.

Jost R., Berkowitz O., Wirtz M., Hopkins L., Hawkesford M.J., and Hell R. (2000). Genomic and functional characterization of the oas gene family encoding O-acetylserine (thiol) lyases, enzymes catalyzing the final step in cysteine biosynthesis in Arabidopsis thaliana. Gene 253, 237-247.

Kavita P. and Burma P.K. (2008). A comparative analysis of green fluorescent protein and beta-glucuronidase protein-encoding genes as a reporter system for studying the temporal expression profiles of promoters. J. Biosci. 33, 337-343.

Kim D.Y., Bovet L., Kushnir S., Noh E.W., Martinoia E., and Lee Y. (2006). AtATM3 is involved in heavy metal resistance in Arabidopsis. Plant Physiol. 140, 922932.

Kim D.Y., Bovet L., Maeshima M., Martinoia E., and Lee Y. (2007). The ABC transporter AtPDR8 is a cadmium extrusion pump conferring heavy metal resistance. Plant J. 50, 207-218.

Kloti A., He X., Potrykus I., Hohn T. and Futterer J. (2002). Tissue-specific silencing of a transgene in rice. Proc Natl Acad USA. 99, 10881-10886.

Kopriva S. (2006). Regulation of Sulfate Assimilation in Arabidopsis and Beyond. Annals of Botany. 97, 479-495.

Korshunova Y.O., Eide D., Clark W.G., Guerinot M.L., and Pakrasi H.B. (1999). The IRT1 protein from Arabidopsis thaliana is a metal transporter with a broad substrate range. Plant Mol. Biol. 40, 37-44. 
Kramer U. (2005). Phytoremediation: novel approaches to cleaning up polluted soils. Curr. Opin. Biotechnol. 16, 133-141.

Kusaba M., Takahashi Y., and Nagata T. (1996). A multiple-stimuli-responsive as-1related element of parA gene confers responsiveness to cadmium but not to copper. Plant Physiol. 111, 1161-1167.

Lee S. and Korban S.S. (2002). Transcriptional regulation of Arabidopsis thaliana phytochelatin synthase (AtPCS1) by cadmium during early stages of plant development. Planta 215, 689-693.

Lee S., Moon J.S., Ko T.S., Petros D., Goldsbrough P.B., and Korban S.S. (2003). Overexpression of Arabidopsis phytochelatin synthase paradoxically leads to hypersensitivity to cadmium stress. Plant Physiol. 131, 656-663.

Leonhardt N. (2006). Genome-wide transcriptome profiling of the early cadmium response of Arabidopsis roots and shoots. Biochimie 88, 1751-1765.

Lescot M., Dehais P., Thijs G., Marchal K., Moreau Y., Van de Peer Y. and Rombauts S. (2002). PlantCARE, a database of plant cis-acting regulatory elements and a portal to tools for in silico analysis of promoter sequences. Nucleic Acids Res. 30, 325327.

Li Z.S., Szczypka M., Lu Y.P., Thiele D.J., and Rea P.A. (1996). The yeast cadmium factor protein (YCF1) is a vacuolar glutathione S-conjugate pump. J. Biol. Chem. 271, 6509-6517.

Logan H.M., Cathala N., Grignon C., and Davidian J.C. (1996). Cloning of a cDNA encoded by a member of the Arabidopsis thaliana ATP sulfurylase multigene family. Expression studies in yeast and in relation to plant sulfur nutrition. J. Biol. Chem. 271, 12227-12233.

Mandel, M. and Higa, A. (1970). Calcium-dependent bacteriophage DNA infection. J. Molec. Biol. 53:159-162.

Maruyama-Nakashita A., Nakamura Y., Watanabe-Takahashi A., Inoue E., Yamaya T., and Takahashi H. (2005). Identification of a novel cis-acting element conferring sulfur deficiency response in Arabidopsis roots. The Plant J. 42, 305-314.

Martin M.N., Tarczynski M.C., Shen B., and Leustek T. (2005). The role of 5'adenylylsulfate reductase in controlling sulfate reduction in plants. Photosynth Res. 86, 309-323.

Martinoia E., Maeshima M., and Neuhaus H.E. (2007). Vacuolar transporters and their essential role in plant metabolism. J. Exp. Bot. 58, 83-102.

McGrath S.P. and Zhao F.J. (2003). Phytoextraction of metals and metalloids from contaminated soils. Curr. Opin. Biotechnol. 14, 277-282. 
Meister A. (1995). Glutathione metabolism. Methods Enzymol. 251, 3-7.

Mendoza-Cozatl D., Loza-Tavera H., Hernandez-Navarro A., and Moreno-Sanchez R. (2005). Sulfur assimilation and glutathione metabolism under cadmium stress in yeast, protists and plants. FEMS Microbiol. Rev. 29, 653-671.

Minglin L., Yuxiu Z., and Tuanyao C. (2005). Identification of genes up-regulated in response to Cd exposure in Brassica juncea L. Gene 363, 151-158.

Momose Y. and Iwahashi H. (2001). Bioassay of cadmium using a DNA microarray: genome-wide expression patterns of Saccharomyces cerevisiae response to cadmium. Environ. Toxicol. Chem. 20, 2353-2360.

Muthukumar B., Yakubov B., and Salt D.E. (2007). Transcriptional activation and localization of expression of Brassica juncea putative metal transport protein BjMTPI. BMC Plant Biol. 7.

Nagy Z., Montigny C., Leverrier P., Yeh S., Goffeau A., Garrigos M., and Falson P. (2006). Role of the yeast ABC transporter Yorlp in cadmium detoxification. Biochimie 88, 1665-1671.

Nascimento C.W.A. and Xing B. (2006). Phytoextraction: A review on enhanced metal availability and plant accumulation. Sci. Agric. (Piricicaba, Braz.). 63, 299-311.

Noctor G. and Foyer C.H. (1998). Ascorbate and Glutathione: Keeping Active Oxygen Under Control. Annu. Rev. Plant Physiol. Plant Mol. Biol. 49, 249-279.

Nriagu J.O. (1979). Global inventory of natural and anthropogenic emissions of trace metals to the atmosphere. Nature $\mathbf{2 7 9}, \mathbf{4 0 9 - 4 1 1 .}$

Ortiz D.F., Ruscitti T., McCue K.F., and Ow D.W. (1995). Transport of metal-binding peptides by HMT1, a fission yeast ABC-type vacuolar membrane protein. J. Biol. Chem. 270, 4721-4728.

Pence N.S., Larsen P.B., Ebbs S.D., Letham D.L., Lasat M.M., Garvin D.F., Eide D., and Kochian L.V. (2000). The molecular physiology of heavy metal transport in the $\mathrm{Zn} / \mathrm{Cd}$ hyperaccumulator Thlaspi caerulescens. Proc. Natl. Acad. Sci. USA. 97, 49564960.

Peterson A.G. and Oliver D.J. (2006). Leaf-targeted phytochelatin synthase in Arabidopsis thaliana. Plant Physiol. Biochem. 44, 885-892.

Phartiyal P., Kim W., Cahoon R., Jez J., and Krishnan H. (2006). Soybean ATP sulfurylase, a homodimeric enzyme involved in sulfur assimilation, is abundantly expressed in roots and induced by cold treatment. Arch. Biochem. Biophys. 450, 20-29. 
Pilon-Smits E.A.H., Zhu Y.L., Sears T. and Terry N. (2000). Overexpression of glutathione reductase in Brassica juncea: Effects on cadmium accumulation and tolerance. Physiologia Plantarum. 110, 455-460.

Pollard J.A. and Baker A.J.M. (1997). Deterrence of herbivory by zinc hyperaccumulation in Thlaspi caerulescens (Brassicaceae). New Phytol. 135, 655-658.

Pomponi M., Censi V., Di Girolamo V., De Paolis A., di Toppi L.S., Aromolo R., Costantino P., and Cardarelli M. (2006). Overexpression of Arabidopsis phytochelatin synthase in tobacco plants enhances $\mathrm{Cd}(2+)$ tolerance and accumulation but not translocation to the shoot. Planta 223, 180-190.

Qi X., Zhang Y., and Chai T. (2007). Characterization of a novel plant promoter specifically induced by heavy metal and identification of the promoter regions conferring heavy metal responsiveness. Plant Physiol. 143, 50-59.

Quinn J.M. and Merchant S. (1995). Two copper-responsive elements associated with the Chlamydomonas Cyc6 gene function as targets for transcriptional activators. Plant Cell 7, 623-628.

Quinn J.M., Kropat J., and Merchant S. (2003). Copper response element and Crr1dependent $\mathrm{Ni}(2+)$-responsive promoter for induced, reversible gene expression in Chlamydomonas reinhardtii. Eukaryot. Cell. 2, 995-1002.

Ramos J., Clemente M.R., Naya L., Loscos J., Perez-Rontome C., Sato S., Tabata S., and Becana M. (2007). Phytochelatin synthases of the model legume Lotus japonicus. A small multigene family with differential response to cadmium and alternatively spliced variants. Plant Physiol. 143, 1110-1118.

Rampias T.N., Fragoulis E.G., and Sideris D.C. (2009). A hybrid-specific polymerase chain reacion-based amplification approach for chromosomal walking. Anal. Biochem. 388, 342-344.

Roje S. (2006). S-adenosyl-L-methionine: Beyond the universal methyl group donor. Phytochemistry. 67, 1686-1698.

Rose A.B. (2004). The effect of intron location on intron-mediated enhancement of gene expression in Arabidopsis. The Plant J. 40, 744-751.

Rotte C. and Leustek T. (2000). Differential subcellular localization and expression of ATP sulfurylase and 5'-adenylylsulfate reductase during ontogenesis of Arabidopsis leaves indicates that cytosolic and plastid forms of ATP sulfurylase may have specialized functions. Plant Physiol. 124, 715-724.

Ryu S.H., Kim Y.H., Kim C.Y., Park S.Y., Kwon S.Y. (2009). Molecular characterization of the sweet potato peroxidase SWPA4 promoter which responds to abiotic stresses and pathogen infection. Physiol. Plantar. 135, 390-399. 
Saeed H.A., Vodkin L.O. and Stromvik M.V. (2008). Promoters of the soybean seed lectin homologues Le2 and Le3 regulate gene expression in vegetatve tissues in Arabidopsis. Plant Sci. 175, 868-876.

Salt D.E., Smith R.D., and Raskin I. (1998). Phytoremediation. Annu. Rev. Plant Physiol. Plant Mol. Biol. 49, 643-668.

Schafer H.J., Haag-Kerwer A., and Rausch T. (1998). cDNA cloning and expression analysis of genes encoding GSH synthesis in roots of the heavy-metal accumulator Brassica juncea L.: evidence for Cd-induction of a putative mitochondrial gammaglutamylcysteine synthetase isoform. Plant Mol. Biol. 37, 87-97.

Schutzendubel A. and Polle A. (2002). Plant responses to abiotic stresses: heavy metalinduced oxidative stress and protection by mycorrhization. J. Exp. Bot. 53, 1351-1365.

Searle P.F., Stuart G.W., and Palmiter R.D. (1987). Metal regulatory elements of the mouse metallothionein-I gene. Experientia Suppl. 52, 407-414.

Seth C.S., Chaturvedi P.K. and Misra V. (2008). The role of phytochelatins and antioxidants in tolerance to $\mathrm{Cd}$ accumulation in Brassica juncea L. Ecotoxicol. Environ. Saf. 71, 76-85.

Shah K. and Dubey R.S. (1995). Effect of Cd on RNA level as well as activity and molecular forms of ribonuclease in growing rice seedlings. Plant Physiol. Biochem. 33, 577.

Shahmuradov, I.A., Gammerman A.J., Hancock J.M., Bramley P.M., and Solovyev V.V. (2003). PlantProm: a database of plant promoter sequences. Nucleic Acids Res. 31, 114-117.

Sharma R.K., Agrawal M., and Marshall F.M. (2008). Heavy metal (Cu, Zn, Cd and $\mathrm{Pb}$ ) contamination of vegetables in urban India: A case study in Varanasi. Environ. Pollut. 154, 254-263

Speiser D.M., Abrahamson S.L., Banuelos G., and Ow D.W. (1992). Brassica juncea Produces a Phytochelatin-Cadmium-Sulfide Complex. Plant Physiol. 99, 817-821.

Takahashi H., Watanabe-Takahashi A., Smith F.W., Blake-Kalff M., Hawkesford M.J., and Saito K. (2000). The roles of three functional sulphate transporters involved in uptake and translocation of sulphate in Arabidopsis thaliana. Plant J. 23, 171-182.

Triglia T., Peterson M.G., and Kemp D. J. (1988). A procedure for in vitro amplification of DNA segments that lie outside the boundaries of known sequences. Nucleic Acids Res. 16, 8186.

Trueba G.A. and Johnson R.C. (1996). Random primed gene walking PCR: a simple procedure to retrieve nucleotide fragments adjacent to known DNA sequences. Biotechniques, 21, 20. 
Vatamaniuk, O.K., Mari, S., Lu, Y. and Rea, P.A. (1999) AtPCS1, a phytochelatin synthase from Arabidopsis: isolation and in vitro reconstitution. Proc. Natl. Acad. Sci. USA. 96, 7110.

Wawrzynski A., Kopera E., Wawrzynska A., Kaminska J., Bal W., and Sirko A. (2006). Effects of simultaneous expression of heterologous genes involved in phytochelatin biosynthesis on thiol content and cadmium accumulation in tobacco plants. J. Exp. Bot. 57, 2173-2182.

Wu X.F., Wang C.L., Xie E.B., Gao Y., Fan Y.L., Liu P.Q., and Zhao K.J. (2009). Molecular cloning and characterization of the promoter for the multiple stress-inducible gene BjCHII from Brassica juncea. Planta. 229, 1231-1242.

Wray J.L., Campbell E.I., Roberts M.A., and Gutierrez-Marcos J.F. (1998). Redefining reductive sulfate assimilation in higher plants: a role for APS reductase, a new member of the thioredoxin superfamily? Chem. Biol. Interact. 109, 153-167.

Xiang C. and Oliver D.J. (1998). Glutathione metabolic genes coordinately respond to heavy metals and jasmonic acid in Arabidopsis. Plant Cell 10, 1539-1550.

Xie X., Lu J., Kulbokas E.J., Golub T.R., Mootha V., Lindbland-Toh K., Lander E.S. and Kellis M. (2005) Systematic discovery of regulatory motifs in human promoters and 3' UTRs by comparison of several mammals. Nature. 434, 338-345.

Yonekura-Sakakibara K., Ashikari T., Tanaka Y., Kusumi T., and Hase T. (1998). Molecular characterization of tobacco sulfite reductase: enzyme purification, gene cloning, and gene expression analysis. J. Biochem. (Tokyo) 124, 615-621.

Yoshimoto N., Takahashi H., Smith F., Yamaya T., and Saito K. (2002). Two distinct high-affinity sulfate transporters with different inducibilities mediate uptake of sulfate in Arabidopsis roots. 29, 465-473.

Yu J., Hu S., Wang J., Wong G.K., Li S. et al. (2002). A draft sequence of the rice genome (Oryza sativa L. ssp. indica). Science. 5, 79-92.

Zhang X., Henriques R., Lin S., Niu Q., and Chua N. (2006). Agrobacterium-mediated transformation of Arabidopsis thaliana using the floral dip method. Nature Protocols 1, $1-6$.

Zhu Y.L., Pilon-Smits E.A. Jouanin L., and Terry N. (1999a). Overexpression of glutathione synthetase in Indian mustard enhances cadmium accumulation and tolerance. Plant Physiol. 119, 73-79.

Zhu Y.L., Pilon-Smits E.A., Tarun A.S., Weber S.U., Jouanin L., and Terry N. (1999b). Cadmium tolerance and accumulation in Indian mustard is enhanced by overexpressing gamma-glutamylcysteine synthetase. Plant Physiol. 121, 1169-1178. 


\section{APPENDIX}

Table 1. Primers designed for amplification of phytochelatin synthase 1 (PS1) and PS2 promoter and coding regions from gDNA

\begin{tabular}{|llll|}
\hline & $\begin{array}{c}\text { Primer } \\
\text { Name }\end{array}$ & $\begin{array}{c}\text { Location } \\
\text { from } \\
\text { ATG }^{a}\end{array}$ & \\
\hline a & PS1-seq7 & -1555 & AACGGAATCATAAGCTTCCAACATG \\
b & PS1-BamHI & -1452 & $\underline{\text { GGATCCTTCCGAAGCGGGAAGAAATATGAGTG }}$ \\
c & PS1-seq1 & -636 & ATAGTGAGCGATGCCCTCCAAACT \\
d & PS1-seq6 & -50 & TCATCCCTCCAAGAAACCTGTCG \\
e & PS1-seq5 & -2 & CTTCCAGTGCGATCTTTGTCAC \\
f & PS1-NcoI & -18 & $\underline{\text { CCATGGTAGCCATTACGACAGGTTTCTTGG }}$ \\
g & PScDNA1 & +499 & GCCATATCTCTCTCAGCATTATAGCCACC \\
h & PS2-seq7 & -1805 & TATTTAATTGTGAGTTGAGACTATTGTACTAATTAAG \\
i & PS2-BamHI & -1459 & $\underline{\text { GGATCCATAGTGGGGTAGAGTGTTGAAT }}$ \\
j & PS2-seq5 & -98 & GAGCGTTGACCTGTGAGAGTCTC \\
k & PS2-seq6 & -41 & TCTCATCACGCCCAAAGTTGAAAG \\
1 & PS2-NcoI & -14 & $\underline{\text { CCATGGCCGTAGCCATTACCACAGGTTTC }}$ \\
\hline
\end{tabular}


Table 2. Primers designed for promoter and coding region amplifications of LAST

\begin{tabular}{|c|c|c|c|}
\hline & Primer Name & $\begin{array}{c}\begin{array}{c}\text { Location } \\
\text { from }\end{array} \\
\text { ATG }^{a} \\
\end{array}$ & Sequence $^{b}$ \\
\hline a & LA-r-BamHI & -3724 & CGGGATCCGAAAGTGCTGGTGTAGTAGGACC \\
\hline b & $\begin{array}{l}\text { LA-r-Tru4- } \\
\text { BamHI }\end{array}$ & -2148 & CGGGATCCTGATGGAGCAGTACGGGAGAGATT \\
\hline $\mathrm{c}$ & $\begin{array}{l}\text { LA-r-Tru3- } \\
\text { BamHI }\end{array}$ & -1485 & CGGGATCCAGCATCAGGGACATGAGAAACCGT \\
\hline d & $\begin{array}{l}\text { LA-r-Tru2- } \\
\text { BamHI }\end{array}$ & -804 & CGGGATCCCTTTGTACTACACACGACCG \\
\hline $\mathrm{e}$ & $\begin{array}{l}\text { LA-r-Tru1- } \\
\text { BamHI }\end{array}$ & -361 & CGGGATCCAACATTTCGTCAACTTGCTCCGCC \\
\hline f & LA-cDNA2 & -18 & GTTCTCTAТСАСССАСТTCAАСАСС \\
\hline $\mathrm{g}$ & $\begin{array}{l}\text { LA-r-pBAR1- } \\
\text { BamHI }\end{array}$ & -33 & GGATCCCATTTTGTTATCTTTGTGGGTGTTGAAGTGGGT \\
\hline $\mathrm{g} *$ & LA-f-NcoI & -9 & CATGCCATGGTCTGCAGCTCTATGCTCATTTTGTT \\
\hline $\mathrm{h}$ & $\begin{array}{l}\text { LA-f-pBAR1- } \\
\text { BamHI }\end{array}$ & 0 & CGGGATCCATGAGCATAGAGCTGCAGAGCC \\
\hline $\mathrm{i}$ & LA-cDNA4 & +1455 & CCAGGGTTTAACCGACCTTCG \\
\hline $\mathrm{j}$ & LA-cDNA3 & +1475 & CGACTTGACCGAGATGTTGACC \\
\hline $\mathrm{k}$ & $\begin{array}{l}\text { LA-f- } \\
\text { pBAR1SacI }\end{array}$ & +2446 & GAGCTCTCAACACATATCGTGCAACGTAGTTC \\
\hline 1 & LA-cDNA1 & +2479 & AACGCTGTCTTAACATACTTGTGC \\
\hline
\end{tabular}

${ }^{a}$ Numbers indicate location of primers with respect to the translational start site of the target gene. Negative numbers represent sites 5' of the translational start site, positive numbers represent sites 3' of the translational start site.

${ }^{b}$ Restriction sites are underlined 
Table 3. Selection and segregation of pLA:GUS A. thaliana transformant lines from $\mathrm{T}_{0}$ to homozygous $\mathrm{T}_{3}$ plants

\begin{tabular}{|c|c|c|c|c|c|c|}
\hline Construct & Length & $\begin{array}{l}\mathbf{T}_{0} \text { Floral } \\
\text { Dipped }\end{array}$ & $\begin{array}{c}\mathbf{T}_{1} \\
\text { Transformants }\end{array}$ & $\begin{array}{c}\text { Segregation } \\
\text { Results with } \\
\text { Probability } \%^{a}\end{array}$ & $\begin{array}{c}\text { Homozygous } \mathrm{T}_{2} \\
\text { plants }\end{array}$ & $\begin{array}{c}\text { GUS } \\
\text { Stained } \mathrm{T}_{3}{ }^{b}\end{array}$ \\
\hline \multirow[t]{18}{*}{ pLA:GUS } & 3771 bp & $\begin{array}{l}\text { (A, B) Late Nov, } \\
07\end{array}$ & A-1 & $20 \%$ & 7,10 & 10-negative \\
\hline & & & B-1 & $30 \%$ & $1,4,8$ & 1-positive \\
\hline & & & B-2 & $50 \%$ & 7 & \\
\hline & & & B-3 & $70 \%$ & $4,5,6,7$ & 7-positive \\
\hline & & & B-4 & $10 \%$ & $3,4,5,6,7,10$ & 7-positive \\
\hline & & & B-5 & $10 \%$ & 6 & \\
\hline & & & B-6 & $80 \%$ & none & \\
\hline & & & B-7 & & & \\
\hline & & & B-8 & $70 \%$ & & \\
\hline & & & B-9 & $80 \%$ & $2,3,4,7,8$ & 2-positive \\
\hline & & & B-10 & $70 \%$ & none & \\
\hline & & & B-11 & $10 \%$ & & \\
\hline & & & B-12 & $10 \%$ & 7, & \\
\hline & & & B-13 & $30 \%$ & 1,5 & \\
\hline & & & B-14 & & & \\
\hline & & & B-15 & $30 \%$ & $2,7,8$ & 7-positive \\
\hline & & & B-16 & $20 \%$ & $1,2,6$ & 7-positive \\
\hline & & & B-17 & $10 \%$ & $3,4,10$ & 10-negative \\
\hline \multirow[t]{19}{*}{$\begin{array}{l}\text { pLA:GUS } \\
\text { Tru } 1\end{array}$} & 436 bp & (A) April, 08 & A-1 & $80 \%$ & $1,7,9$ & 9-negative \\
\hline & & & $A-2$ & $50 \%$ & $4,8,9,10$ & 9-positive \\
\hline & & & A-3 & $90 \%$ & $2,7,10$ & 10-positive \\
\hline & & & $A-4$ & False Positive & & \\
\hline & & & A-5 & False Positive & & \\
\hline & & & A- 6 & False Positive & & \\
\hline & & & A-7 & False Positive & & \\
\hline & & & A-8 & False Positive & & \\
\hline & & & A-9 & $20 \%$ & 1,8 & 10-negative \\
\hline & & & A-10 & $5 \%$ & 6 & 6-positive \\
\hline & & & A-11 & False Positive & & \\
\hline & & & A-12 & $30 \%$ & 3,7 & 3-negative \\
\hline & & & A-13 & $30 \%$ & 7 & 7-negative \\
\hline & & & A-14 & $50 \%$ & $1,6,7,10$ & 6-positive \\
\hline & & & A-15 & $10 \%$ & 1,10 & \\
\hline & & & A-16 & False Positive & & \\
\hline & & & A-17 & $100 \%$ & 6,10 & 10-negative \\
\hline & & & A-18 & False Positive & & \\
\hline & & & A-19 & False Positive & & \\
\hline
\end{tabular}




\begin{tabular}{|c|c|c|c|c|c|c|}
\hline \multirow[t]{15}{*}{ 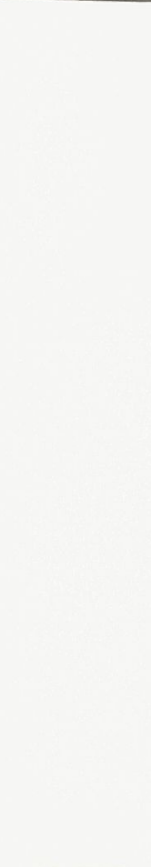 } & 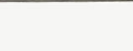 & (B) May 15, 08 & B-1 & $30 \%$ & 5,6 & 5-Positive \\
\hline & & & B-2 & False Positive & & \\
\hline & & & B-3 & $80 \%$ & & \\
\hline & & & B-4 & $30 \%$ & $3,5,8$ & 8-Negative \\
\hline & & & B-5 & False Positive & & \\
\hline & & & B-6 & $80 \%$ & $2,5,6$ & 6-Negative \\
\hline & & & B-7 & $30 \%$ & & \\
\hline & & & B-8 & $50 \%$ & $2,7,10$ & \\
\hline & & & B-9 & $50 \%$ & $1,3,5,10$ & \\
\hline & & & B-10 & $5 \%$ & $2,3,7,9$ & \\
\hline & & & B-11 & $80 \%$ & 1 & \\
\hline & & & B-12 & $80 \%$ & 2,4 & \\
\hline & & & B-13 & $50 \%$ & & \\
\hline & & & B-14 & $50 \%$ & 9 & \\
\hline & & & B-15 & $80 \%$ & $3,5,7$ & \\
\hline \multirow[t]{11}{*}{$\begin{array}{c}\text { pLA:GUS } \\
\text { Tru } 2\end{array}$} & $890 \mathrm{bp}$ & (A) April, 08 & A1-A20 & False Positive & & \\
\hline & & & $A-21$ & $10 \%$ & & \\
\hline & & (B) May 09 & B-1 & $30 \%$ & & \\
\hline & & & B-2 & $80 \%$ & & \\
\hline & & & B-3 & $20 \%$ & & \\
\hline & & & B-4 & $80 \%$ & & \\
\hline & & & B-5 & $50 \%$ & & \\
\hline & & & B-6 & $30 \%$ & & \\
\hline & & & B-7 & $50 \%$ & & \\
\hline & & & B-8 & $70 \%$ & & \\
\hline & & & B-9 & $80 \%$ & & \\
\hline \multirow[t]{12}{*}{$\begin{array}{c}\text { PLA:GUS } \\
\text { Tru } 3\end{array}$} & $1535 \mathrm{bp}$ & (A) May 15, 08 & $A-1$ & $10 \%$ & $7,8,9$ & 7-positive \\
\hline & & & $A-2$ & $90 \%$ & & \\
\hline & & & $A-3$ & $10 \%$ & & \\
\hline & & & $A-4$ & False Positive & & \\
\hline & & & $A-5$ & False Positive & & \\
\hline & & & $A-6$ & $80 \%$ & $1,3,6,7,10$ & 6-positive \\
\hline & & & A-7 & $50 \%$ & 1 & 1-negative \\
\hline & & (B) May 22,08 & B-1 & $50 \%$ & $3,4,6$ & 6-Positive \\
\hline & & & B-2 & $1 \%$ & $1,3,6,7,8$ & 8-Negative \\
\hline & & & B-3 & & & \\
\hline & & & B-4 & $50 \%$ & 1 & 1-negative \\
\hline & & & B-5 & $80 \%$ & $1,2,3$ & 3-positive \\
\hline
\end{tabular}




\begin{tabular}{|c|c|c|c|c|c|c|}
\hline & & & B-6 & $50 \%$ & $1,2,4$ & 2-positive \\
\hline & & & B-7 & $20 \%$ & $1,2,3,4,5,6,7,8$ & 3-negative \\
\hline & & & B-8 & $10 \%$ & $5,6,9$ & 3-negative \\
\hline & & & B-9 & & & \\
\hline & & & B-10 & $5 \%$ & 3,9 & 9-Positive \\
\hline & & & B-11 & $80 \%$ & $3,4,8$ & 3-Negative \\
\hline & & & B-12 & $80 \%$ & 3,4 & 3-Negative \\
\hline & & & B-13 & $50 \%$ & & \\
\hline & & & B-14 & $70 \%$ & $2,4,5,7$ & 2-Negative \\
\hline & & & B-15 & $50 \%$ & $1,7,8$ & 1-Positive \\
\hline & & & B-16 & $50 \%$ & $1,2,5,6,9,10$ & 5-Positive \\
\hline & & & B-17 & $10 \%$ & 3 & \\
\hline & & & B-18 & $80 \%$ & $3,4,8$ & \\
\hline & & & B-19 & $80 \%$ & $2,4,6,8,10$ & \\
\hline $\begin{array}{c}\text { pLA:GUS } \\
\text { Tru } 4\end{array}$ & 2198 bp & (A) April, 08 & $A-1$ & $90 \%$ & 1,7 & 7-positive \\
\hline & & & $A-2$ & $30 \%$ & $5,8,9$ & 8-positive \\
\hline & & & $A-3$ & $10 \%$ & $2,5,6,7,8$ & 2-negative \\
\hline & & & $A-4$ & False Positive & & \\
\hline & & & $A-5$ & $30 \%$ & 4, & 4-negative \\
\hline & & & A-6 & False Positive & & \\
\hline & & & $A-7$ & False Positive & & \\
\hline & & & $A-8$ & False Positive & & \\
\hline & & & $A-9$ & False Positive & & \\
\hline & & & $A-10$ & $80 \%$ & $1,8,10$ & 1-negative \\
\hline & & & B-1 & $50 \%$ & $2,7,8$ & 7-positive \\
\hline & & & B-2 & False Positive & & \\
\hline & & & B-3 & False Positive & & \\
\hline & & & B-4 & $70 \%$ & 7 & 7-negative \\
\hline & & & B-5 & False Positive & & \\
\hline & & & B-6 & $80 \%$ & 1 & 1-positive \\
\hline & & & $A-17$ & $5 \%$ & 6,8 & 8-Positive \\
\hline & & & A-18 & $10 \%$ & & \\
\hline & & & A-19 & $90 \%$ & 4,9 & 4-Negative \\
\hline
\end{tabular}

${ }^{a}$ Segregation results were determined by comparing the survived:dead ratio to the expected 3:1 ratio by Chi squared test.Percents represent the probability of obtaining the given ratio.

The numbers correspond to the $\mathrm{T}_{3}$ seed tested 